\title{
QUASI-ISOMETRIC MEASURES AND THEIR APPLICATIONS ${ }^{1}$
}

\author{
BY P. MASANI
}

1. Introduction . . . . . . . . . . . . . . . . . . . . . . . . 427

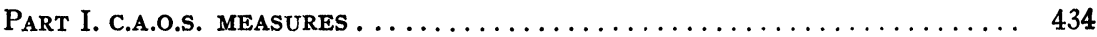

2. C.a.o.s. measures and integration ..................... 434

3. A new approach to $L_{2}$-transform theory .................. 440

4. The Hilbert transform $\ldots \ldots \ldots \ldots \ldots \ldots \ldots \ldots \ldots \ldots \ldots \ldots \ldots, 441$

5. The Watson transform .............................. 443

6. The Fourier-Plancherel transform .................... 446

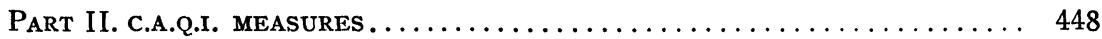

7. On situations with multiplicity exceeding $1 \ldots \ldots \ldots \ldots \ldots \ldots \ldots, 448$

8. $W$-to-HC c.a.q.i. measures........................... 449

9. On the Hilbert space $L_{2}(\Lambda, \mathbb{B}, M ; W) \ldots \ldots \ldots \ldots \ldots \ldots \ldots \ldots \ldots . \ldots \ldots 4$

10. Integration with respect to c.a.q.i. measures................. 464

11. Theory for locally compact semigroups and groups............ 486

12. A new approach to representation theory................ 497

13. The Fourier-Plancherel transform for vectorial functions........... 498

14. Spectral representations......................... 503

15. Linear stationary causal systems and Cooper's theorem ........... 511

16. Unfinished work ............................. 521

REFERENCES . . . . . . . . . . . . . . . . . . . . . . . . . . . 525

\section{Introduction}

This paper is devoted to certain uses of integration theory which emerge when the measures involved are vector- or operator-valued. These uses, as yet generally unfamiliar, are significant in three ways:

(1) They yield explicit formulations for many of the representation theorems of functional analysis;

An expanded version of an address delivered before the Cincinnati meeting of the Society by invitation of the Committee to Select Hour Speakers for Western Sectional Meetings, April 18, 1969; received by the editors October 29, 1969.

$A M S$ Subject Classifications. Primary 2825, 2850, 4250, 4428, 4615, 4730; Secondary $4251,4430,4645,6050$.

Key Words and Phrases. Measures and bases, orthogonally scattered measures, transition from the discrete to the continuous, random measures, stochastic integration, representation theory, wandering subspace, eigen-packets, pseudo-eigenfunction expansions, general $L_{2}$-transform theory, Hilbert transform, Watson transform, Fourier-Plancherel transform, nonnegative hermitian measures, quasi-isometric measures, stationary measures over semigroups and groups, spectral representations, stationary causal systems, Stone's Theorem, Cooper's Theorem, Imprimitivity Theorem.

${ }^{1}$ This work was supported by the National Science Foundation under Grant GP 7808. 
(2) They provide a substitute for the notion of basis in analytical situations in which bases do not appear naturally;

(3) They offer a means for the transition from the discrete to the continuous, and for subsumation of both continuous and discrete theories in one framework.

The remarks in 1.1-1.3 are intended to elucidate these three roles of integration.

1.1. RePresentation theORY. Let $X, Y$ be compact Hausdorff spaces, $C(X)$ be the set of all complex-valued, continuous functions on $X$, and $\mathscr{M}(Y)$ be the set of regular, c.a. complex-valued bounded measures on the $\sigma$-algebra of Borel subsets of $Y . C(X)$ and $\operatorname{Tr}(Y)$ are Banach spaces under the usual norms. The statement

(1) Given $X, \exists Y \ni \mathfrak{M}(Y)$ is isometrically isomorphic to $C(X)^{*}$,

is then correct, but it is not as informative and explicit as the conclusion of the Riesz Representation Theorem, viz.

$$
Y=X \text {, and the isometric isomorphism } \Sigma \text { on } \operatorname{Tr}(X) \text { onto }
$$

$$
\begin{aligned}
& C(X)^{*} \text { is given by } \\
& \forall \mu \in \mathfrak{M}(X), \quad\{\Sigma(\mu)\} f=\int_{X} f(x) \mu(d x), \quad f \in C(X) .
\end{aligned}
$$

This illustrates how integration helps in making an isomorphism explicit. There is much value in carrying out such explication, for it suggests that the representation theorem stems from a generalized notion of convexity and so belongs to a rather universal part of mathematics. For instance, on writing $\varepsilon_{x}$ for the evaluation at $x$, so that $\varepsilon_{x}(f)=f(x),(2)$ becomes

$$
\Sigma(\mu)=\int_{X} \mu(d x) \varepsilon_{x} .
$$

If we transplant the measure $\mu$ from $X$ to the set $\varepsilon_{X}=\left\{\varepsilon_{x}: x \in X\right\}$ of evaluations over $X$, i.e. consider $\mathfrak{N}\left(\varepsilon_{X}\right)$ in place of $\mathfrak{T}(X)$, we get from (2), proceeding heuristically,

$$
\Sigma(\mu)=\int_{\mathcal{E}_{X}} \mu\left(d \varepsilon_{x}\right) \cdot \varepsilon_{x}, \quad \mu \in \mathfrak{M}\left(\varepsilon_{X}\right)
$$

where $\varepsilon_{x}$ is a dummy variable ranging over $\varepsilon_{X}$. But what (3) says is that 
This strongly suggests that the evaluations $\varepsilon_{x}$ may be extreme points of a certain part of the unit sphere in $C(X)^{*}$, and that our theorem may be a corollary of the Krein-Milman Theorem in its Choquet integral formulation, cf. [40]. ${ }^{2}$

We contend that integration with respect to the right kind of vector- and operator-valued measures likewise renders explicit many other representation theorems of analysis, among them Stone's Theorem on the unitary representation of a 1.c.a. group; results on unitary transforms on $L_{2}$-spaces, especially the Fourier-Plancherel duality between a 1.c.a. group and its dual; Cooper's Theorem on the isometric representation of the semigroup $[0, \infty)$, and the closely related translational and spectral representations of prediction theory, scattering theory and of invariant subspaces of the Hardy class $\mathrm{H}_{2}$; several results in eigenfunction expansions and perturbation theory; Mackey's Imprimitivity Theorem which generalizes the Weyl-Stone-von Neumann statement of the quantum mechanical commutation relations, etc.

1.2. Measures as substitutes for bases. Let $X$ be a (complex) Banach space. We say that $\left(\xi_{\lambda}, \lambda \in \Lambda\right)$, where $\xi_{\lambda} \in X$, is a basis for $X$, iff $^{3}$

$$
\forall x \in X, \quad \exists \mid\left(c_{\lambda}\right)_{\lambda \in \Lambda} \ni \quad c_{\lambda} \in C \text { and } \sum_{\lambda \in \Lambda} c_{\lambda} \xi_{\lambda} \text { cgs. } \&=x .
$$

If an analytic problem involves a concrete Banach or Hilbert function-space $X$ and a basis attaches itself naturally to the problem, we could of course use it and expand functions in $X$ in terms of the basic functions. But there are situations in which there is no such natural basis. A typical example is the Fourier-Plancherel theory for $L_{2}(R)$. $L_{2}(R)$ is separable and there are plenty of known o.n. bases, but all are irrelevant for the purposes of the FP theory. The basis we want is precisely the one which is not there, viz. $\left(e_{\lambda}, \lambda \in R\right)$ where $e_{\lambda}(t)=e^{i \lambda t}$; obviously $e_{\lambda} \notin L_{2}(R)$.

Several solutions have been proposed to deal with such situations in which there are no relevant bases, but the one which seems most natural to the writer is to seek a $\sigma$ - or $\delta$-ring $B$ over the parameter space $\Lambda$ and an $X$-valued, c.a. measure $\xi$ on $B$ such that

\footnotetext{
2 Unfortunately, the Riesz Thm. is used in the current treatments of the Choquet theory, so at present it cannot be derived from Choquet's Thm. But the writer feels that this is a blemish of the current definition and treatment of the Choquet integral, and that a different but equivalent definition with a modified treatment would yield the Riesz Thm. as a corollary.

3 " $\exists \mid$..." means "there exists a unique ...". $\boldsymbol{C}$ denotes the complex number field. $\boldsymbol{R}$ will denote the real number field.
} 


$$
\forall x \in X, \quad \exists \mid \phi(\cdot) \text { on } \Lambda \text { to } \mathbf{C} \ni \int_{\Lambda} \phi(\lambda) \xi(d \lambda) \text { exists } \&=x \text {. }
$$

We might call such a $\xi$ a basic measure for $X$.

The only case in which a complete theory of such measures $\xi$ exists is the one in which $X$ is a Hilbert space $\mathfrak{H}$, and $\xi$ is akin to an orthogonal basis in $\mathcal{H}$. Such measures $\xi$ are said to be orthogonally scattered. There is also a fairly well-advanced theory of such measures for inflated $\mathcal{H}^{q}$, inflation meaning that we consider $\mathcal{H}^{q}$ with $q \times q$ Gram matricial inner products, and $p \times q$ matrices in place of scalars. Again with $X=\mathfrak{H}$, a certain amount of work has been done on nonorthogonally scattered measures by probabilists interested in stochastic processes. But for $X \neq \mathfrak{F}$, we do not know of any progress in this direction.

From the work done so far it appears that for a useful theory of basic measures the following conditions should be met:

(1) The parameter space $\Lambda$ should be a subset of a locally compact abelian group with Haar measure $\mu$, and $B$ should be the $\delta$-ring $B_{\mu}$ of Borel sets of finite Haar measure, and moreover $\xi \ll \mu$;

(2) Uniqueness should be interpreted as "uniqueness a.e., $\mu$ ";

(3) Although $\xi \ll \mu$, $\xi$ should not be the indefinite (Bochner) integral of $\mu$, i.e. the Radon-Nikodým derivative $d \xi / d \mu$ should not exist. ${ }^{4}$

1.3. TRANSITION FROM THE DISCRETE TO THE CON'TINUOUS. Imagine that time is discrete, i.e. all instances of time are integers. A particle undergoes a random displacement $y_{n}$ in $R^{3}$ at each instant $n \geqq 0$, these successive displacements being independent. We interpret each $y_{n}$ as a function on a probability space $(\Omega, F, P)$ and assume that $y_{n} \in L_{2}\left(\Omega, F, P ; R^{3}\right)$. The stochastic process (SP) $\left(x_{n}, n \geqq 0\right)$, where $x_{n}=\sum_{0}^{n} y_{k}$ may then be called a free random walk in $R^{3}$.

We now take time to be continuous, and ask for the notion corresponding to a free random walk. Clearly we must consider variates $x_{t}(\cdot)$, where $t \in[0, \infty)$, and to exclude pathological cases demand that $x_{t} \rightarrow x_{s}$ in the mean as $t \rightarrow s$. It is then clear that $x_{t}$ cannot be a continuous sum of independent random variates $y_{s}, 0 \leqq s \leqq t$. We are therefore obliged to define a continuous time random walk by stipulating the behavior of its increments, viz. as a $\operatorname{SP}\left(x_{t}, t \in[0, \infty)\right)$ such that

" Roughly speaking, the RN Thm. should fail. This is because we want our measure $\xi$ to be "linearly independent" in the sense that $\int_{\Lambda} \phi(\lambda) \xi(d \lambda)=\int_{\Lambda} \psi(\lambda) \xi(d \lambda) \Rightarrow \phi=\psi$ a.e. $(\mu)$. The Bochner integral of a vector-valued function w.r.t. a scalar measure does not satisfy such a condition. But the existence of $d \xi / d \mu$ would entail this condition, and so produce a contradiction. 


$$
x_{b}-x_{a} \in L_{2}\left(\Omega, F, P ; R^{3}\right), \quad 0 \leqq a<b
$$

and

$$
x_{b}-x_{a} \& x_{d}-x_{c} \text { are indep. when }(a, b] \|(c, d] \text {. }
$$

Such a process $\left(x_{t}(\cdot), t \in[0, \infty)\right)$ is said to be of independent increments. The set-function $\xi$ defined by

$$
\xi_{(a, b]}=x_{b}-x_{a}
$$

is clearly a measure on the prering $P$ of all open-closed subintervals $(a, b]$ of $[0, \infty)$ having values in the Hilbert space $L_{2}\left(\Omega, \mathfrak{F}, P ; R^{3}\right)$-a so-called random measure.

We see here how the transition from discrete to continuous time took us from a vector-valued point-function $x$ to a vector-valued measure $\xi$.

For the especially important type of SP of independent increments known as the Brownian motion the associated vector-valued measure $\xi$ has the property

$$
\left|\xi_{(a, b]}\right|_{2}^{2}=\text { const. }(b-a) \text {. }
$$

By rescaling, the constant can be made 1 . By (4), $\xi$ is absolutely continuous with respect to Lebesgue measure $\mu$, but the occurrence of the square on the LHS shows that the RN derivative $d \xi / d \mu$ will not exist. Also this $\xi$ is unbounded, and even after maximal extension will be defined only on the $\delta$-ring of sets of finite Lebesgue measure.

Another problem in the transition from the discrete to the continuous concerns the concept of wandering subspace due to Halmos $\left[14\right.$, p. 102]. Let $\left(S^{n}, n=\right.$ integer $\left.\geqq 0\right)$ be a semigroup of isometries on a Hilbert space $\mathcal{H C}$ into itself. A subspace $W$ of $\mathcal{H C}$ is said to be wandering with respect to this semigroup, iff

$$
S^{m}(W) \perp S^{n}(W) \quad \text { when } m \neq n \text {. }
$$

Now let $\left(S_{t}, t=\right.$ real $\left.\geqq 0\right)$ be a semigroup of isometries, which to avoid pathologies we assume to be strongly continuous. When can a subspace $W$ of $\operatorname{HC}$ be said to wander with respect to this semigroup? Again there is no obvious answer, and we could dismiss the question as being meaningless. But if we pursue this question with the attitude we took to the random walk, we find that canonically attached to the operator-valued point-function $S_{t}$ is an operator-valued measure $T_{(a, b]}$ and that in a very natural sense $W$ may be said to wander, iff

$$
T_{(a, b]}(W) \perp T_{(c, d]}(W) \text { whenever }(a, b] \|(c, d] \text {. }
$$


In fact,

$$
T_{(a, b]}=(1 / \sqrt{ } 2)\left\{S_{b}-S_{a}-\int_{a}^{b} S_{t} d t\right\} .
$$

We can show that $(1 / \sqrt{ }(b-a)) T_{(a, b]}$ is an isometry when restricted to $W$, so that

$$
T(a, b]^{*} T(a, b]=(b-a) I \quad \text { on } W .
$$

We therefore call $T$ a quasi-isometric measure. The similarity between (5) and the result (4), rescaled to make the constant 1 , is far from fortuitous.

1.4. HistoricAl AND BIBLIOGRAPHICAL REMARKS. The idea of an infinite-dimensional vector-valued measure can be traced back at least to 1909 when Hellinger in his fundamental paper $[\mathbf{1 5}, \S 5]$ introduced the idea of eigendifferential, and what subsequently has been called an eigenpacket or more of ten wave packet, for the study of differential operators with a continuous spectrum. The eigenpacket is in essence a Hilbert space-valued measure, cf. [16, 10.4]. The first systematic treatment of such measures occurs, however, in Wiener's epoch-making paper [49] of 1923 on the mathematical analysis of the Einstein-Smoluchovski theory of the Brownian movement. In this originated the concept of a process of independent increments and the related notions of random measure and stochastic integration, cf. [10, p. 426]. In the late 20's spectral (projection-valued) measures were introduced by Stone and von Neumann for the study of linear operators on Hilbert spaces. While this important advance led to greater use of Hilbert space-valued measures, it focussed attention solely on the bounded ones defined on $\sigma$-algebras. As a result, the important class of vector-valued measures which are governed by Haar measure and are consequently unbounded (cf. 1.2(2) \& 1.3(4) et seq.), did not receive adequate attention. In recent years the study of multivariate stochastic processes has led to the consideration of orthogonally scattered measures in inflated Hilbert spaces $\mathfrak{H C}^{q},[34]$, [44], [29]. Also Loève, Cramer, Rosanov and others interested in stochastic processes have considered nonorthogonally scattered measures with values in $\mathfrak{H C},[23],[9],[42]$.

The close connection between probability theory and random measures on the one hand and $L_{2}$-transform theory and harmonic analysis on the other is clearly discernible from the writings of Wiener, Bochner and others [50], [39], [5]. But the observation that the underlying measure and integration theories are identical in the 
two situations and that vector-valued measures can therefore be used to systematize and simplify $L_{2}$-transform theory seems to be due to the writer [30], [32]. The writer also introduced the notion of quasiisometric measure in the course of finding a Wold Decomposition for continuous parameter isometric semigroups [28], and he observed that these measures have a wider utility [31]. This work was stimulated by the time-domain techniques developed jointly with J. B. Robertson in [33]. These observations will be amplified in the sequel.

We should also mention some work on an ancillary problem that confronts us. Let $C L\left(W^{\prime}, W\right)$ be the Banach space of continuous linear operators on a Hilbert space $W^{\prime}$ to a Hilbert space $W$, and let $M$ be a countably additive, $W$-to- $W$, nonnegative hermitian operator-valued measure on a $\sigma$-ring $B$ over a space $\Lambda$. The problem is to define the space

$$
\mathfrak{L}_{2}=L_{2}\left(\Lambda, \leftrightarrow, M ; C L\left(W^{\prime}, W\right)\right)
$$

in such a way that it becomes a Hilbert space. In short, we need the operatorial generalization of the classical Riesz-Fischer Theorem for $L_{2}(R)$. This ancillary problem was settled by Rosenberg [44] and Rosanov [43] for finite-dimensional $W^{\prime}$ and $W$. The case when $W^{\prime}=\mathbf{C}$ and $W$ is infinite-dimensional is discussed by Kuroda [21] and the case $W^{\prime}=W$ where both are infinite-dimensional by Mandraker and Salehi in an unpublished report [26].

The work described in the last three paragraphs has a markedly Hilbertian flavor. A related development in a non-Hilbertian context is the theory of vector-valued measures and integration due to Bartle, Dunford and Schwartz, which is presented in volume I of their book $[11],{ }^{5}$ and the vectorial extension of the classical duality theorems for $C(X)$ and $L_{p}$, due to I. Singer [46], [47], Bartle, Dunford and Schwartz [2], Bogdanowicz [6], Mizel and Sudaresan [36] and others. We shall not be concerned with this important work in the present paper. In the future, when more is known concerning basic measures in Banach spaces, it may become possible to embrace this work along with ours in a single general theory. It may also become possible to secure an even greater unification by considering vector-valued and operator-valued measures on abstract Boolean $\delta$-rings instead of $\delta$-rings of subsets of a given set, a suggestion for which we are grateful to Professor Garrett Birkhoff.

1.5. The SCOPE of This PAPER. Part I is devoted to orthogonally

${ }^{5}$ Their theory so far pertains to bounded measures defined on $\sigma$-algebras, and so excludes most of the measures we need. 
scattered measures. In $\$ 2$ we recapitulate the central aspects of the theory, developed more fully in [30], which are needed for the applications. In $\$ 3$ we suggest a new orthogonally-scattered measuretheoretic procedure to deal with unitary transforms on $L_{2}$-spaces in an explicit way, and illustrate it by application to the Hilbert transform ( $\$ 4)$ and the Watson transform $(\$ 5)$. In $\$ 6$ we allude to the explicit formulation of the general Fourier-Plancherel transform already given fully in [32].

In Part II we turn to quasi-isometric operator-valued measures. In $\$ 7$ we explain how such measures take over the role of orthogonally scattered measures in situations involving Hilbert spaces in which a certain multiplicity exceeds 1 . We then study such measures in $\$ 8$. $\S 9$ is devoted to the ancillary question of the definition and completeness of the space $L_{2}(\Lambda, \Theta, M ; W)$ of functions on $\Lambda$ to a Hilbert space $W$ which are "square-integrable" with respect to a $W$-to- $W$, nonnegative, hermitian operator-valued measure $M$. Integration with respect to quasi-isometric measures over arbitrary spaces $\Lambda$ is treated in detail in $\$ 10$. The important special case in which $\Lambda$ is a locally compact semigroup or group, and the ensuing concept of "stationarity" are taken up in $\$ 11$.

$\$ \$ 12-15$ are devoted to applications of quasi-isometric measures. In $\$ 12$ we propose a new quasi-isometric measure-theoretic procedure to deal with representations of Hilbert spaces in an explicit way. We illustrate this procedure by application to vectorial FourierPlancherel theory $(\$ 13)$, spectral representation theorems $(\$ 14)$, Cooper's Theorem on the isometric representations of the semigroup $[0, \infty)$, and the analysis of linear, stationary, causal systems ( $\$ 15)$. In $\$ 16$ we refer to other aspects of our theory and to some unfinished work on the explicit formulation of Mackey's Imprimitivity Theorem, and of theorems on perturbations and pseudo-eigenfunction expansions.

\section{PART I. C.A.O.S. MEASURES}

\section{C.a.o.s. measures and integration}

The theory of Hilbert-space valued, orthogonally scattered measures has been expounded rather fully in [30]. It will therefore suffice to recall only the definition and the few fundamental results needed to exemplify the role of such measures as substitutes for orthogonal bases, and as a foundation for the treatment of unitary transforms on $L_{2}$-spaces.

2.1. Definition. Let Je be a complex Hilbert space. We say that $\xi$ is 
an J-valued, countably additive, orthogonally scattered (c.a.o.s.) measure over $(\Lambda, B, \mu)$, iff

(i) $B$ is a $\sigma$-ring over a space $\Lambda$,

(ii) $\mu$ is a nonnegative, $\sigma$-finite, c.a. measure on $B$,

(iii) $\xi$ is an J-valued function on the $\delta$-ring ${ }^{6}$

$$
\Theta_{\mu} \underset{d}{=}\{B: B \in \Theta \& \mu(B)<\infty\},
$$

such that

$$
\forall A, B \in B_{\mu}, \quad(\xi(A), \xi(B))=\mu(A \cap B) .
$$

$\mu$ is called the nonnegative measure of $\xi$.

It follows trivially that if $\xi$ is an $\mathcal{H}$-valued, c.a.o.s. measure over $(\Lambda, B, \mu)$, then $\xi$ is countably additive and orthogonally scattered, i.e.

$$
\begin{gathered}
\forall k \geqq 1, \quad B_{k} \in B_{\mu}, \quad B_{k} \text { are } \|^{7} \quad \& \bigcup_{1}^{\infty} B_{k} \in B_{\mu} \\
\Rightarrow \sum_{1}^{\infty} \xi\left(B_{k}\right) \text { converges unconditionally to } \xi\left(\bigcup_{1}^{\infty} B_{k}\right) ; \\
A, B \in B_{\mu} \quad \& A \| B \Rightarrow \xi(A) \perp \xi(B) .
\end{gathered}
$$

The converse also holds, $[30,1.8]$. This justifies our use of the appellation "c.a.o.s." in 2.1.

2.2. Definition. Let $\xi$ be an $\mathfrak{H}$-valued, c.a.o.s. measure over $(\Lambda, \mathbb{B}, \mu)$. Then
$(a)^{8}$
$\Im\left\{\xi\left(B_{\mu}\right)\right\}, \quad$ i.e. $\subseteq\left\{\xi(A): A \in \mathbb{B}_{\mu}\right\}$

is called the subspace of $\xi$ and denoted by $\varsigma_{\xi}$.

(b) In case $\varsigma_{\xi}=\mathfrak{H}$ we call $\xi$ an $\mathfrak{H}$-basic, c.a.o.s. measure (for now the "differentials" of $\xi$ act like a basis for $\mathfrak{H C}$ ).

Barring trivial cases c.a.o.s. measures $\xi$ have infinite total variation; indeed for many $\xi,|\xi|(B)=\infty$ whenever $\mu(B)>0$. Also, although obviously $\xi$ is absolutely continuous relative to its nonnegative measure $\mu$, there is in general no $\mathcal{H}$-valued Radon-Nikodym derivative $d \xi / d \mu,[30,4.4]$. Despite these complications, the theory is very simple, thanks to the strong nexus between $\xi$ and $\mu$, viz. $|\xi(B)|^{2}=\mu(B), \forall B \in B_{\mu}$.

- So-called because it is closed under countable intersections. The symbol $=_{d}$ means "equals by definition."

7 The symbol || means disjoint.

${ }^{8} \forall A \subseteq \mathcal{H}, \subseteq(A)={ }_{d}$ the (least, closed, linear) subspace spanned by $A$. 
There is an elegant concept of integration of functions $\phi \in$ $L_{2}(\Lambda, \Theta, \mu)$ with respect to an $\mathcal{H}$-valued, c.a.o.s. measure $\xi$ over $(\Lambda, \Theta, \mu)$. The integral is first defined for $\Theta_{\mu}$-simple functions $\phi$, and then by a limiting process for any $\phi$ in $L_{2}(\Lambda, \beta, \mu),[30,5.4,5.6]$. The crucial property of the integral is given in the following theorem $[30,5.9]$ :

2.3. IsOMORPHISM THEOREM. Let $\xi$ be an FC-valued, c.a.o.s. measure over $(\Lambda, \Theta, \mu)$. Then the correspondence

$$
S: \phi \rightarrow \int_{\Lambda} \phi(\lambda) \xi(d \lambda)
$$

is an isometry on $L_{2}(\Lambda, \mathbb{B}, \mu)$ onto $\mathcal{S}_{\xi} \subseteq \mathcal{H}$. Thus, every such $\xi$ carries with it two Hilbert spaces, $S_{\xi}$ and $L_{2}(\Lambda, ®, \mu)$, isomorphic under the natural correspondence $S$.

Theorem 2.3 shows, for instance, that

$$
\begin{aligned}
\left(\int_{\Lambda} \phi(\lambda) \xi(d \lambda), \int_{\Lambda} \psi(\lambda) \xi(d \lambda)\right) & =(\phi, \psi)_{\mu}=\int_{d} \phi(\lambda) \overline{\psi(\lambda)} \mu(d \lambda), \\
& \left|\int_{\Lambda} \phi(\lambda) \xi(d \lambda)\right|^{2}=|\phi|_{\mu}^{2}=\int_{d}|\phi(\lambda)|^{2} \mu(d \lambda) .
\end{aligned}
$$

Let $\xi$ be an $\mathcal{H}$-valued, c.a.o.s. measure over $(\Lambda, \beta, \mu)$ and $P_{\xi}$ be the projection on $\mathcal{H C}$ onto $\S_{\xi}$. Then by Thm.2.3 to each $x$ in $\mathcal{H C}$ corresponds a function $\phi_{x}$ in $L_{2}(\Lambda, \Theta, \mu)$ such that

$$
P_{\xi}(x)=\int_{\Lambda} \phi_{x}(\lambda) \xi(d \lambda) .
$$

This $\phi_{x}$ can be expressed in terms of $x$ and $\xi$ by a Radon-Nikodým derivative as shown in $[30,5.10]$; but for the purposes of this paper it will suffice to give only the more cogent form this result takes when $\Lambda$ is a Besicovitch space with respect to $\mu$ (2.6 below). We first define this notion:

2.5. Definition. Let (i) $\Lambda$ be a Hausdorff space,

(ii) B be a $\sigma$-ring over $\Lambda$ which includes the family of Borel sets,

(iii) $\mu$ be a nonnegative, $\sigma$-finite, c.a., Borel measure ${ }^{9}$ on $ß$. We call $(\Lambda, B)$ a Besicovitch space with respect to $u$, iff $\Lambda$ has a special family of precompact open neighborhoods $N_{\lambda}$ of points $\lambda$ in $\Lambda$ having the following property: $\forall$ complex-valued c.a. measures $\nu$ such that the Hahn extension of the total variation $|\nu|$ is a Borel measure on $\mathbb{B}$,

${ }^{9}$ I.e. such that $\mu(C)<\infty, \forall$ compact $C \subseteq \Lambda$ 


$$
\frac{D \nu}{D \mu}(\lambda)=\lim _{N_{\lambda} \rightarrow \lambda} \frac{\nu\left(N_{\lambda}\right)}{\mu\left(N_{\lambda}\right)} \quad \text { exists a.e. }(\mu) \text { on } \sigma(\mu) \subseteq \Lambda,
$$

where $\sigma(\mu)$ is the spectrum of $\mu . D \nu / D \mu$ is called the Besicovitch derivative of $v$ with respect to $u$.

Let $\nu=\nu_{a}+\nu_{b}, \nu_{a} \ll \mu$, be the Lebesgue decomposition of $\nu$ relative to $\mu$. Writing $d \nu_{a} / d \mu$ for the Radon-Nikodým derivative, it follows from Besicovitch's basic theorem [3] that

$$
D \nu / D \mu=\text { a version of } d \nu_{a} / d \mu \text {. }
$$

As one may surmise from our remarks after $2.2, D \xi / D \mu$ does not exist in general for a c.a.o.s. measure $\xi$ over $(\Lambda, \beta, \mu),[30,6.5]$. Nevertheless, we have the following theorem $[30,6.7]$ :

2.6. Projection theorem (for Besicovitch $\Lambda$ ). Let (i) $\xi$ be an HC-valued measure over $(\Lambda, B, \mu)$ where $(\Lambda, B)$ is a Besicovitch space with respect to (the $\sigma$-finite, Borel measure) $\mu$;

(ii) $P_{\xi}$ be the projection on $\mathrm{FC}$ onto $s_{\xi}$. Then

$$
\forall x \in \mathfrak{H C}, \quad P_{\xi}(x)=\int_{\Lambda} \lim _{N_{\lambda} \rightarrow\{\lambda\}}\left(x, \frac{\xi\left(N_{\lambda}\right)}{\mu\left(N_{\lambda}\right)}\right) \xi(d \lambda),
$$

the integrand being always a function in $L_{2}(\Lambda, \beta, \mu)$.

The last limit can be brought inside the inner product when and only when $\mu\{\lambda\}>0$, i.e. $\{\lambda\}$ is an atom of $\mu$, as shown in $[30,6.6]$. Let us compare the last equation with the familiar formula

$$
\forall x \in \mathcal{H}, \quad P_{\xi}(x)=\sum_{n=1}^{\infty}\left(x, \frac{\xi_{n}}{\left|\xi_{n}\right|^{2}}\right) \xi_{n}
$$

where $\left(\xi_{n}\right)_{1}^{\infty}$ is any orthogonal sequence in $\mathcal{H C}$, and $P_{\xi}$ is the projection onto the subspace spanned by the $\xi_{n}, n \geqq 1$. Clearly the equation in 2.6 is a generalization of (1), and reduces to (1) when the measure $\xi$ is purely atomic and concentrated on the set of positive integers. Thus, Thm.2.6 may well be regarded as a generalized Pythagorean Theorem.

In the applications we are usually concerned with the case in which the Hilbert space $\mathcal{H C}$ consists of complex-valued functions which are $L_{2}$ over a measure space $(\Omega, F, \nu)$. A slight modification of our notation is expedient in this case:

2.7. Notation. Let $\xi$ be an $L_{2}(\Omega, F, \nu)$-valued, c.a.o.s. measure over $(\Lambda, B, \mu)$, cf. 2.1. Then since the value of $\xi$ at $B$, where $B \in \Theta_{\mu}$, 
is a function on $\Omega$, we shall denote this value by $\xi_{B}$ and not by $\xi(B)$. We can then write equations such as

$$
\forall A, B \in \Theta_{\mu}, \quad \int_{\Omega} \xi_{A}(\omega) \overline{\xi_{B}(\omega)} \nu(d \omega)=\left(\xi_{A}, \xi_{B}\right)_{\nu}=\mu(A \cap B) .
$$

Our integral will be written $\int_{\Lambda} \phi(\lambda) \xi_{d \lambda}$ and not $\int_{\Lambda} \phi(\lambda) \xi(d \lambda)$.

A distinctive feature of the $L_{2}$ spaces is their possession of a basic c.a.o.s. measure distinguishable from all others by its simplicity $[30,9.3]$ :

2.8. TRIVIality. Let (i) $\nu$ be a nonnegative, $\sigma$-finite, c.a. measure on a $\sigma$-ring $\mathfrak{F}$ over $\Omega$, so that $\mathcal{F}=\sigma\left(\mathscr{F}_{\nu}\right)$ where

$$
\mathscr{F}_{\nu}=\{E: \quad E \in \mathfrak{F} \& \nu(\mathfrak{F})<\infty\},
$$

and $\sigma\left(\mathfrak{F}_{\nu}\right)$ denotes the $\sigma$-ring generated by $\mathfrak{F}_{\nu}$;

(ii) $\forall E \in \mathscr{F}_{\nu}, \chi_{E}$ be the indicator-function of $E$. Then $\chi$ is a $L_{2}(\Omega, F, \nu)$ basic, c.a.o.s. measure over $(\Omega, \mathcal{F}, \nu)$. Moreover,

$$
\forall f \in L_{2}(\Omega, \mathcal{F}, \nu), \quad f=\int_{\Omega} f(\omega) \chi_{d \omega} .
$$

We shall call the $\chi$ given in 2.8 the indicator-measure basis for $L_{2}(\Omega, \mathcal{F}, \nu)$. It is the obvious analogue of the familiar basis: $(1,0$, $0, \cdots),(0,1,0, \cdots), \cdots$ for the space $l_{2}$.

In 1933 Watson [48] studied the general transforms on $L_{2}[0, \infty)$ which have since been given his name. In 1934 Bochner [4] simplified and extended Watson's treatment, retaining however the use of point-functions. The following is the c.a.o.s. measure-theoretic reformulation of his result for any $L_{2}$ spaces $[30,9.7(a)]$ :

2.9. Duality theorem (Bochner). Let (i) for $i=1,2, \mu_{i}$ be a nonnegative, $\sigma$-finite, c.a. measure on a $\sigma$-ring $\AA_{i}$ over a space $\Lambda_{i}$, and

$$
\beta_{\mu_{i}}=\left\{B: B \in \Theta_{i} \& \mu_{i}(B)<\infty\right\} ;
$$

$$
\mathfrak{H C}_{i}=L_{2}\left(\Lambda_{i}, \Theta_{i}, \mu_{i}\right)
$$

(iii) $\eta$ be a $\mathfrak{H C}_{2}$-valued, c.a.o.s. measure over $\left(\Lambda_{1}, \AA_{1}, \mu_{1}\right)$, $\xi$ be a $\mathfrak{H C}_{1^{-}}$ valued, c.a.o.s. measure over $\left(\Lambda_{2}, \mathrm{~B}_{2}, \mu_{2}\right)$;

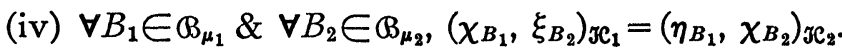

Then $\mathrm{S}_{\xi}=\mathfrak{H C}_{1}, \mathrm{~S}_{\eta}=\mathfrak{H C}_{2} ;$ i.e. $\xi$ and $\eta$ are $\mathfrak{H}_{1}$-basic and $\mathfrak{H C}_{2}$-basic c.a.o.s. measures, respectively.

This theorem yields the following corollary $[30,9.5,9.6,9.7(\mathrm{~b})]$ : 
2.10. Corollary. Let (i)-(iv) be as in 2.9, and let

$$
\forall f \in \mathcal{H}_{1}, \quad V(f)=\int_{\Lambda_{1}} f\left(\lambda_{1}\right) \eta_{d \lambda_{1}} .
$$

Then (a) $V$ is a unitary operator on $\mathfrak{K}_{1}$ onto $\mathfrak{H}_{2}$,

$$
\forall g \in \mathfrak{H}_{2}, \quad V^{*}(g)=\int_{\Lambda_{2}} g\left(\lambda_{2}\right) \xi_{d \lambda_{2}} .
$$

The following result is a converse of 2.9 and 2.10:

2.11. Corollary. Let (i) and (ii) be as in 2.9 and let

(iii) $V$ be a unitary operator on $\mathfrak{H}_{1}$ onto $\mathfrak{H}_{2}$,

(iv) $\forall B_{1} \in B_{\mu_{1}} \& \forall B_{2} \in B_{\mu_{2}}, \quad \eta_{B_{1}}=V\left(\chi_{B_{1}}\right) \& \xi_{B_{2}}=V^{*}\left(\chi_{B_{2}}\right)$.

Then the conditions 2.9 (iii) and (iv) hold.

Finally, we must mention the case in which $\Lambda$ is a subinterval of $R$. A natural correspondence then exists between set-functions and point-functions, concerning which there are two useful results:

2.12. Lemma. Let (i) $\Lambda$ be any subinterval of $\boldsymbol{R}$ with left endpoint $a,^{10}$

(ii) $x(\cdot)$ be a function on $\Lambda$ to $\mathfrak{F}$ such that in case $a \notin \Lambda, \lim _{t \rightarrow a+} x(t)$ exists,

$$
l= \begin{cases}x(a), & a \in \Lambda, \\ \lim _{t \rightarrow a+} x(t), & a \notin \Lambda .\end{cases}
$$

Then the following conditions are equivalent:

$(\alpha) x(\cdot)$ has orthogonal increments, i.e.

$$
\begin{aligned}
& \forall a, b, c, d \in \Lambda, \quad a<b \leqq c<d \Rightarrow x(b)-x(a) \perp x(d)-x(c) \\
& (\beta) \quad \exists f(\cdot) \text { on } \Lambda \exists \forall s, t \in \Lambda, \quad(x(s)-l, x(t)-l)=f(\min \{s, t\}) .
\end{aligned}
$$

We omit the easy proof of this result. On taking $s=t$ in $(\beta)$, we see that actually

$$
f(\cdot)=|x(\cdot)-l|^{2} .
$$

It also easily follows, cf. $[30,8.2]$, that when $(\alpha)$ or $(\beta)$ holds, $f$ is monotone increasing on $\Lambda$ and $x(\cdot)$ has left- and right-limits $x(t \pm)$, $\forall t \in \Lambda$. On combining 2.12 with the theorem $[30,8.6]$ that a function with orthogonal increments generates a c.a.o.s. measure, we get the following theorem:

\footnotetext{
$10 a$ need not be in $\Lambda$ and may equal $-\infty$.
} 
2.13. EXTENSION THEOREM. Let the conditions 2.12(i)-(iii) and $(\alpha)$ or $(\beta)$ hold, and let

$$
B=\{B: B \text { is a Borel subset of } \Lambda\} .
$$

Then $\exists$ a unique nonnegative, $\sigma$-finite, c.a. measure $\mu$ on $\Theta$ and $\exists a$ unique $\mathfrak{H}$-valued c.a.o.s. measure $\xi$ over $(\Lambda, \AA, \mu)$ such that

$\forall(a, b] \subseteq \Lambda, \mu(a, b]=f(b+)-f(a+) \& \xi(a, b]=x(b+)-x(a+)$.

\section{A new approach to $L_{2}$-transform theory}

Hitherto the Fourier-Plancherel, Hankel, Hilbert and other unitary transforms on $L_{2}$-spaces have been defined by improper or singular integrals (1.i.m.'s, Cauchy p.v.'s, $(d / d x) \int$, etc.). Now let $V$ be any unitary transformation on one $L_{2}$ space onto another $L_{2}$ space. Then by $2.11 V\left(\chi_{(\cdot)}\right), V^{*}\left(\chi_{(\cdot)}\right)$, where $\chi_{(\cdot)}$ are indicator measures over the two $L_{2}$ spaces, are basic c.a.o.s. measures related as in the Duality Thm.2.9. Hence by 2.10 all such transformations are definable by (proper) integrals of $L_{2}$-valued c.a.o.s. measures. This suggests a new approach to $L_{2}$ transform theory, concordant with the view expressed in 1.2, based on the following procedure:

3.1. Procedure. (i) From the data of the problem define a pair of $L_{2}$-valued set-functions $\xi, \eta$;

(ii) Show that they satisfy the conditions of the Duality Thm.2.9, and hence are basic c.a.o.s. measures; (Cor. 2.10 then ensures that the resulting transformation $V$ is unitary.)

(iii) Use the Projection Thm.2.6 to express $V(f)$ as an improper or singular integral, and so identify $V$ or $V^{*}$ with the classical transform.

This approach has several advantages. (1) It is shorter than the traditional one in which there is no appeal to a general theory, and consequently the same sort of reasoning has to be repeated in a special context in each instance. (2) It permits the exploitation of the theory of c.a.o.s. measured integration (e.g. of theorems on indefinite integration, substitution, integration by parts, etc., cf. $[30, \S \S 5-9]$, to find new connections between different transforms, and so secure a better organization of the theory. (3) It brings $L_{2}$-transform theory closer to certain parts of probability theory, the only difference that remains between the two being that in the latter our measures have values in

$$
L_{2}(\Omega, \sigma \text {-alg., Probab. meas.), }
$$

whereas in the former their values are in 
$L_{2}$ (l.c.a. group or part, Borel family, Haar meas.).

We shall illustrate the new approach by considering the Hilbert, Watson and Fourier-Plancherel transforms ( $\$ \$ 4-6)$.

\section{The Hilbert transform}

Following Procedure 3.1, we start by defining a pair of set-functions $\xi, \eta$ :

$$
\begin{aligned}
& -\xi_{(a, b]}(\lambda)=\eta_{(a, b]}(\lambda)=\frac{1}{\pi} \log \left|\frac{b-\lambda}{a-\lambda}\right|, \\
& \text { where } a, b, \lambda \in R \quad \& \quad a<b \quad \& \quad a \neq \lambda \neq b .
\end{aligned}
$$

Now an elementary analytical argument shows that

$$
\forall h, k \in R, \quad \log |\cdot+h|-\log |\cdot+k| \in L_{2}(R) .
$$

It follows that

$$
\forall(a, b] \subset R, \quad \eta_{(a, b]} \in L_{2}(R),
$$

and a routine calculation yields its $L_{2}$-norm:

$$
\left|\eta_{(a, b]}\right|_{2}^{2}=\frac{A}{\pi}(b-a), \quad \text { where } A=\int_{0}^{\infty}\left(\log \left|\frac{1+\lambda}{1-\lambda}\right|\right)^{2} d \lambda .
$$

By (4.1) and (1), $\eta$ is a $L_{2}(R)$-valued, finitely additive measure on the prering $\rho$ of bounded subintervals $(a, b]$ of $R$, and by (4.2) its nonnegative measure $\mu(\cdot)=$ const. Leb., and is therefore c.a. But it is a triviality that a $\mathcal{F}$-valued, f.a. measure $\eta$ on $P$, for which $\left|\eta_{(\cdot)}\right|_{2}^{2}$ is c.a. and $\left(\eta_{A}, \eta_{B}\right) \in R$ is c.a.o.s. on $\odot$. Thus, $\eta$ is a $L_{2}(R)$-valued c.a.o.s. measure on $P$ and its nonnegative measure is Lebesgue. Using the same letter for the Hahn extension to the Borel family $B$, it follows, cf. $[30,2.5]$, that

$$
\xi, \eta \text { are } L_{2}(R) \text {-valued, c.a.o.s. meass. over }\left(R, \Theta, \frac{A}{\pi} \text { Leb. }\right) \text {. }
$$

To complete step (ii) of Procedure 3.1, we next check whether condition (iv) of the Duality Thm.2.9 is satisfied. For $B_{1}=(a, b]$ and $B_{2}=(c, d]$ this condition is

$$
-\int_{a}^{b} \frac{1}{\pi} \log \left|\frac{d-\lambda}{c-\lambda}\right| d \lambda=\int_{c}^{d} \frac{1}{\pi} \log \left|\frac{b-\lambda}{a-\lambda}\right| d \lambda,
$$

and its truth follows immediately from the triviality ${ }^{11}$

${ }^{11}$ Which holds unrestrictedly if we set $0^{\circ}={ }_{d} 1$. 


$$
\int_{a}^{b} \log \left|\frac{d-\lambda}{c-\lambda}\right| d \lambda=\log \left\{\frac{|b-d|^{b-d}|a-c|^{a-c}}{|b-c|^{b-c}|a-d|^{a-d}}\right\} .
$$

The condition 2.9(iv) thus holds on the prering $\beta$ and thence by Hahn extension on the $\delta$-ring $B_{0}$. From 2.9 we thus obtain

4.3. Theorem (CAuchy-Hilbert Basic measure). The Hahnextensions of the set-functions $\xi, \eta$ defined in (4.1) are $L_{2}(R)$-basic, c.a.o.s. measures over $(R, B,(A / \pi)$ Leb.), where the constant $A$ is as in (4.2).

It follows from Cor.2.10 that

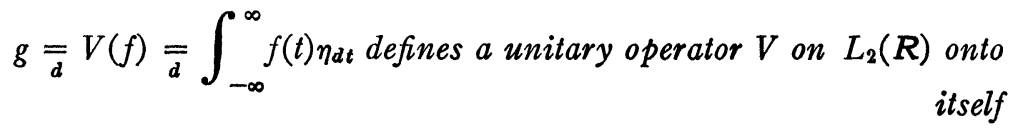

$$
\& \quad f=V^{*}(g)=\int_{-\infty}^{\infty} g(\lambda) \xi_{d \lambda}
$$

To identify this $V$ with the Hilbert transform as defined classically, we appeal of course to the Projection Thm.2.6 (step (iii) of Procedure 3.1). From the second equation in (4.4) and Thm.2.6

$$
\begin{aligned}
g(\lambda) & =\lim _{h \rightarrow 0}\left(f, \frac{1}{2 h} \xi_{(\lambda-h, \lambda+h)}\right) \\
& =\lim _{h \rightarrow 0} \frac{1}{2 h \pi} \int_{-\infty}^{\infty} f(t) \log \left|\frac{\lambda-h-t}{\lambda+h-t}\right| d t, \quad \text { by (4.1) } \\
& =\lim _{h \rightarrow 0}\left\{\frac{1}{2 h \pi}\left(\int_{-\infty}^{\lambda-h}+\int_{\lambda+h}^{\infty}\right) \cdots+\frac{1}{2 h \pi} \int_{\lambda-h}^{\lambda+h} \cdots\right\} \\
& =\lim _{h \rightarrow 0}\{\mathrm{I}+\mathrm{II}\}, \quad \text { say. }
\end{aligned}
$$

We now apply Lebesgue's theorems on convergence and differentiation in a straightforward way, and show that

$$
\lim _{h \rightarrow 0} \text { II }=0 \text {, }
$$

and

$$
\lim _{h \rightarrow 0} \mathrm{I}=\lim _{h \rightarrow 0}\left(\int_{-\infty}^{\lambda-h}+\int_{\lambda+h}^{\infty}\right) \frac{f(t)}{t-\lambda} d t=\mathrm{P} . \mathrm{V} \cdot \int_{-\infty}^{\infty} \frac{f(t)}{t-\lambda} d t .
$$

Hence, 


$$
g(\lambda)=(V f)(\lambda)=\text { P.V. } \int_{-\infty}^{\infty}(f(t) /(t-\lambda)) d t, \quad \text { a.e.(Leb.). }
$$

Thus $V$ is indeed the Hilbert transform.

\section{The Watson transform}

In his important paper [48] Watson set out to answer the following question. Let

$w$ be a complex-valued, ${ }^{12}$ Borel measurable function

$$
\text { on }[0, \infty) \ni w(\cdot) / \mathrm{I}(\cdot) \in L_{2}[0, \infty) .{ }^{13}
$$

What further conditions must $w(\cdot)$ satisfy in order that it yield a transformation $V$ such that

(i) $V$ is unitary on $L_{2}[0, \infty)$ onto itself

(ii) $g=\frac{\bar{d}}{d}(f)$ is such that

$$
g(x)=\frac{d}{d x} \int_{0}^{\infty} \frac{\bar{w}(x y)}{y} f(y) d y, \text { a.e. on }[0, \infty),
$$

(iii) the relation between $g$ and $f$ is reciprocal, i.e.

$$
f(x)=\frac{d}{d x} \int_{0}^{\infty} \frac{w(x y)}{y} g(y) d y, \quad \text { a.e. on }[0, \infty) \text {. }
$$

Watson obtained the following remarkable answer:

5.3. WATSON'S THEOREM. The n.\&s.c. for the existence of a transformation $V$ satisfying the conditions in (5.2) is that

$$
\forall a, b \geqq 0, \quad \int_{0}^{\infty} \frac{w(a y) \bar{w}(y b)}{y^{2}} d y=\min \{a, b\} .
$$

Proof (following Procedure 3.1). For notational simplicity we shall suppose that $w(0)=0$. Define for $b>a \geqq 0$,

$$
\overline{\eta_{(a, b]}(\lambda)}=\xi_{d a, b]}(\lambda)=\frac{w(b \lambda)-w(a \lambda)}{\lambda}, \quad \lambda>0,
$$

and

$$
\forall t \in[0, \infty), \quad X(t) \underset{d}{=} w(t \cdot) / I(\cdot)
$$

12 Actually, Watson took $w$ to be real-valued.

${ }_{13} I(\cdot)$ is the identity function: $I(x)=x, x \geqq 0$. 
Then clearly $X(\cdot)$ is a function on $[0, \infty)$ to $L_{2}[0, \infty)$ and

$$
\xi_{(a, b]}=X(b)-X(a), \quad 0 \leqq a<b .
$$

The condition (5.3.1) tells us that

$$
(X(a), X(b))=\min \{a, b\}, \quad 0 \leqq a<b,
$$

i.e. that the condition $2.12(\beta)$ holds with $l=w(0)=0$ and $f(t)=t$, $t \geqq 0$. This $f(\cdot)$ is continuous on $[0, \infty)$ and so therefore is $X(\cdot)$. Hence assuming that (5.3.1) holds, it follows at once from Thm.2.13 that

$$
\xi, \eta \text { are } L_{2}[0, \infty) \text {-valued c.a.o.s. meass. over }([0, \infty), \infty, \mathrm{Leb} .)
$$

where $B$ denotes the $\sigma$-ring of Borel subsets of $[0, \infty)$, and $\xi, \eta$ denote the Hahn extensions of the measures in (5.4).

To complete step (ii) of Procedure 3.1, we next check whether the condition (iv) of the Duality Thm.2.9 is met. For $B_{1}=(a, b]$ and $B_{2}=(c, d]$, this condition becomes

$$
\int_{a}^{b} \frac{\bar{w}(d \lambda)-\bar{w}(c \lambda)}{\lambda} d \lambda=\int_{c}^{d} \frac{\bar{w}(b \lambda)-\bar{w}(a \lambda)}{\lambda} d \lambda .
$$

Now,

LHS $=\int_{0}^{b} \frac{\bar{w}(d \lambda)}{\lambda} d \lambda-\int_{0}^{a} \frac{\bar{w}(d \lambda)}{\lambda} d \lambda-\int_{0}^{b} \frac{\bar{w}(c \lambda)}{\lambda} d \lambda+\int_{0}^{a} \frac{\bar{w}(c \lambda)}{\lambda} d \lambda$.

On substituting $\lambda=b u / d, a u / d, b u / c, a u / c$ in the four integrals respectively, we get four other integrals which when combined obviously yield the RHS. Thus the condition 2.9 (iv) holds on the prering $P$, and thence by extension on the $\delta$-ring of Borel sets of finite Lebesgue measure. From Thm.2.9 and Cor.2.10, we now conclude that

(5.6) $\xi, \eta$ are $L_{2}[0, \infty)$-basic c.a.o.s. meass. over $([0, \infty), ®$, Leb. $)$ and that

$$
\begin{aligned}
& g=V(f)=\int_{d}^{\infty} f(t) \eta_{d t} \text { defines a unitary operator on } L_{2}[0, \infty) \text { onto } \\
& \text { itself, }
\end{aligned}
$$

Finally, we come to step (iii) of Procedure 3.1. From the second eqn. in (5.7) and the Projection Thm.2.6, 


$$
\begin{aligned}
g(\lambda) & =\lim _{h \rightarrow 0}\left(f, \frac{1}{2 h} \xi(\lambda-h, \lambda+h]\right) \quad \text { a.e. (Leb) on }[0, \infty) \\
& =\lim _{h \rightarrow 0} \int_{0}^{\infty} f(t) \frac{\bar{w}\{(\lambda+h) t\}-\bar{w}\{(\lambda-h) t\}}{2 h t} d t \\
& =\lim _{h \rightarrow 0} \frac{1}{2 h}\left\{\int_{0}^{\infty} f(t) \frac{\bar{w}\{(\lambda+h) t\}}{t} d t-\int_{0}^{\infty} f(t) \frac{\bar{w}\{(\lambda-h) t\}}{t} d t\right\} \\
& =\frac{d}{d \lambda} \int_{0}^{\infty} f(t) \frac{\bar{w}(\lambda t)}{t} d t, \quad \text { a.e. (Leb.) on }[0, \infty) .
\end{aligned}
$$

Similarly from the first eqn. in (5.7) and the Projection Thm.2.6,

$$
f(t)=\frac{d}{d t} \int_{0}^{\infty} g(\lambda) \frac{w(t \lambda)}{\lambda} d \lambda, \quad \text { a.e. (Leb.) on }[0, \infty) .
$$

We have thus established that

$$
\text { (5.3.1) } \Rightarrow \text { (5.2), under the hyp. (5.1). }
$$

To prove the converse implication, let (5.2) hold, and take $f=\chi_{(0, a]}$ in (5.2)(ii). Then

$$
\begin{aligned}
V\left(\chi_{(0, a]}\right)(x) & =\frac{d}{d x} \int_{0}^{a} \frac{\bar{w}(x y)}{y} d y, & & \text { a.e. (Leb.), } \\
& =\frac{d}{d x} \int_{0}^{x} \frac{\bar{w}(a t)}{t} d t, & & (\text { put } t=x y / a), \\
& =\frac{\bar{w}(a x)}{x}, & & \text { a.e. (Leb.) }
\end{aligned}
$$

by the Lebesgue differentiation theorem. Thus,

$$
\begin{aligned}
\int_{0}^{\infty} \frac{w(a \lambda) \bar{w}(b \lambda)}{\lambda^{2}} d \lambda & =\left(V\left(\chi_{(0, a]}\right), V\left(\chi_{(0, b]}\right)\right) & \\
& =\left(\chi_{(0, a]}, \chi_{(0, b]}\right) & \text { by }(5.2)(\mathrm{i}) \\
& =\operatorname{Leb} .((0, a] \cap(0, b]) & \text { by }(2.8) \\
& =\min \{a, b\} . &
\end{aligned}
$$

This completes the proof of the converse.

Disregarding aspects of Watson's theorem which are subsumed under the results of $\$ 2$, and therefore now redundant from our standpoint, we may describe his contribution as the discovery of the following remarkable result: 
5.8. TheOREM (WATSON'S CONDITION FOR BASIC MEASURE). Let

(i) $w(\cdot)$ be a complex-valued, Borel-measurable function on $[0, \infty)$ such that $w(\cdot) / I(\cdot) \in L_{2}[0, \infty)$ and $w(0)=0$,

$$
\text { for } 0 \leqq a<b, \quad \xi_{(a, b]}(\lambda)=(w(b \lambda)-w(a \lambda)) / \lambda, \quad \lambda>0 .
$$

Then the Hahn extension of $\xi$ is a $L_{2}[0, \infty)$-basic c.a.o.s. measure over $([0, \infty), \beta, L e b)$, iff

$$
\forall a, b>0, \quad \int_{0}^{\infty} \frac{w(a \lambda) \bar{w}(b \lambda)}{\lambda^{2}} d \lambda=\min \{a, b\} .
$$

\section{The Fourier-Plancherel transform}

As Bochner [4] has shown, a slightly amended form of Watson's Theorem 5.3 works for $L_{2}[a, b]$ for any $a, b$ such that $-\infty \leqq a<b \leqq \infty$. Now take

$$
(a, b)=R, \quad \text { and } \quad w(\lambda)=\left(e^{i \lambda}-1\right) / \lambda, \quad \lambda \neq 0, \quad w(0)=0 .
$$

Then, as shown e.g. in $[41$, p. 294], $w$ satisfies the amended condition corresponding to (5.3.1), and so yields a unitary operator $V$ on $L_{2}(R)$ onto itself, which is seen to be the FP transform.

Alternatively, we can develop FP theory on a c.a.o.s. measuretheoretic basis following Procedure 3.1. This is clear for the group $R$, since we have only to carry out the demonstration given in $\$ 5$ with slight changes, taking $w$ to be the function given in (6.1). The corresponding treatment for an arbitrary locally compact, abelian (1.c.a.) group, which is much harder, has been given in detail in [32]. Here we need indicate only its very salient features.

Let $\mathcal{F}=L_{2}(X, \beta, \mu)$, where $X$ is an (additive) 1.c.a. group with Borel family $B$ and (regular) Haar measure $\mu$, and let $\hat{\mathcal{C}}=L_{2}(\hat{X}, \hat{A}, \hat{\mu})$, where $\hat{X}$ is the (multiplicative) character group of $X$ with Borel family $\hat{\beta}$ and dual Haar measure $\hat{\mu} \cdot{ }^{14}$ Let $\widehat{B}_{0}, \hat{\otimes}_{0}$ denote the $\delta$-rings consisting of Borel sets of finite Haar measure, and $[x, \alpha]$ stand for $\alpha(x)$, where $x \in X, \alpha \in \hat{X}$. Following Procedure 3.1, we first define two set functions $\xi, \eta$ :

$$
\begin{aligned}
& \forall B \in \hat{\beta}_{0} \& \forall x \in X, \quad \xi_{\hat{B}}(x)=\int_{\hat{B}}[x, \alpha] \hat{\mu}(d \alpha), \\
& \forall B \in \beta_{0} \& \forall \alpha \in \hat{X}, \quad \eta_{B}(\alpha)=\int_{B} \overline{[x, \alpha]} \mu(d x) .
\end{aligned}
$$


To carry out step (ii) of Procedure 3.1, we prove that $\xi, \eta$ are 3 -valued and $\hat{\mathcal{C}}$-valued c.a.o.s. measures over $(\hat{X}, \hat{\mathcal{O}}, \hat{\mu})$ and $(X, \beta, \mu)$, respectively, satisfying the condition (iv) of the Duality Thm.2.9:

$$
\forall B \in \mathbb{B}_{0} \& \forall \hat{B} \in \hat{\mathbb{B}}_{0}, \quad\left(\chi_{B}, \xi_{\hat{B}}\right)_{\mathcal{H C}}=\left(\eta_{B}, \chi \hat{B}\right)_{\mathfrak{H C}}
$$

This is a rather intricate matter for which we have to invoke the Pontryagin Duality Theorem as well as the Fourier Inversion Theorem for $L_{1}(X, B, \mu)$. We then get the following crucial result [32, 4.8]:

6.4. Theorem (Fourier-Plancherel basic measures). Let $\xi, \eta$ be as in (6.2). Then

(a) $\quad \xi$ is a $\mathcal{H}$-basic, c.a.o.s. measure over $(\hat{X}, \hat{\Re}, \hat{\mu})$,

(b) $\quad \eta$ is a $\hat{\mathcal{H}}$-basic, c.a.o.s. measure over $(X, \beta, \mu)$.

This theorem is a generalization of the well-known result that the characters of the group $X$ provide an orthonormal basis for the space $\mathcal{H}=L_{2}(X, \Theta, \mu)$ when $X$ is compact, cf. [38, p. 424]. What Thm.6.4 says is that when $X$ is locally compact it is the "character packets" $\xi_{\hat{B}}, \hat{B} \in \hat{O}_{0}$, defined in (6.2), which provide a basic c.a.o.s. measure for $\mathcal{H C}$.

Thm.6.4 allows us of course to define the FP transform $V$ on $\mathfrak{H C}$ to $\hat{H C}$ explicitly by the formula

$$
\forall f \in \mathfrak{H}, \quad f=V(f)=\int_{d} f(x) \eta_{d x} .
$$

It follows from Cor.2.10, that $V$ is a unitary operator on $\mathfrak{H C}$ onto $\hat{\mathfrak{C}}$, and that

$$
\forall g \in \hat{\mathfrak{H}}, \quad \tilde{g}=V^{*}(g)=\int_{\hat{X}} g(\alpha) \xi_{d \alpha} .
$$

From $6.2,6.4,(6.5)$ we can deduce all the known results of FP theory. For groups which are Besicovitch spaces with respect to their Haar measures, cf. $[12, \$ 2]$, we can also get some new results from our Projection Thm.2.6, cf. [32, \$5].

For nonabelian, locally compact groups, preliminary investigations by D. J. Patil and E. Schwandt suggest that a c.a.o.s. measure-theoretic treatment will work for the FP theory at least in the unimodular case. Because of the breakdown of duality, only the c.a.o.s. measure $\eta$ seems to survive, and its values now lie in the tensor product of certain $L_{2}$-spaces, i.e. in a space of Hilbert-Schmidt operators.

To recover the FP theory for the group $R$ from the general theory, we have only to note that $\hat{R}$ is isomorphic to, and therefore identifi- 
able with $R$, and that a convenient choice of Haar measure is $(1 / \sqrt{ } 2 \pi)$ (Lebesgue measure) rather than Lebesgue measure itself, as it is the former which is self-dual. With this choice of Haar measure, the definition (6.2) of the FP measures $\xi, \eta$ reduces to

$$
\forall B \in \Theta_{0} \& \forall t \in R, \quad \xi_{B}(t)=\frac{1}{\sqrt{ } 2 \pi} \int_{B} e^{i \hbar \lambda} d \lambda=\bar{\eta}_{B}(t),
$$

and the entire theory can be built on this basis.

\section{PART II. C.A.Q.I. MEASURES}

\section{On situations with multiplicity exceeding 1}

In the situation considered in Part I a certain underlying Hilbert space $W$ happens to be $\boldsymbol{C}$. There are analytic situations in which the corresponding spaces $W$ have dimension $q>1$. As instances we may cite the Fourier-Plancherel theory for functions with values in a Hilbert space $W$, and the representation theorems of Stone and Cooper, cf. 1.1. $q$ turns out to be the multiplicity of a certain associated spectral measure; therefore we speak of it as a multiplicity.

To deal with these " $q>1$ situations" in the same explicit manner as the " $q=1$ situations" of Part I, we have to take instead of our $\mathfrak{K C}$ valued, c.a.o.s. measure a $W$-to-Fe, c.a.q.i. measure having the crucial property of quasi-isometry (q.i.), which we shall define in §8. Just as the c.a.o.s. measure $\xi$ has attached to it a nonnegative real-valued measure $\mu$, so the c.a.q.i. measure $T(\cdot)$ has attached to it a $W$-to- $W$, nonnegative, hermitian operator-valued measure $M(\cdot)$. In the causal situations considered so far in [28], [31], $M(\cdot)=\mu(\cdot) I$, where $\mu(\cdot)$ is a nonnegative, real-valued measure and $I$ is the identity operator on $W$. But recently we have felt the need to admit other less-trivial kinds of $W$-to- $W$, nonnegative, hermitian operator-valued measures $M$ as well, in order to reach other (noncausal) situations, and to secure a wider unification. ${ }^{15}$

Just as integration with respect to c.a.o.s. measures makes explicit an isomorphism

$$
\Sigma: L_{2}(\Lambda, B, \mu ; C) \rightarrow \mathfrak{K C}
$$

${ }^{15}$ In fact, problems have already emerged which suggest that even this wider framework is inadequate, and that we should take instead of $W$ a tensor product $W^{\prime} \circ W$, where $W^{\prime}$ is another Hilbert space, keeping $M(\cdot)$ as a $W$-to- $W$, nonnegative, hermitian operator-valued measure. But in this paper we shall only consider $W$, i.e. from the more general standpoint consider the specialization $W^{\prime}=\boldsymbol{C}$. 
so integration with respect to c.a.q.i. measures makes explicit an isomorphism

$$
\Sigma: L_{2}(\Lambda, ß, M ; W) \rightarrow \mathfrak{H C}
$$

A preliminary question is as to when $L_{2}(\Lambda, \beta, M ; W)$ is a Hilbert space. We shall comment on this in $\$ 9$, and consider integration in $\$ 10$.

\section{8. $W$-to-HC c.a.q.i. measures}

In this section

(i) $\rho$ is a prering over an arbitrary set $\Lambda$;

(ii) $W$ \& Je are Hilbert spaces over $\mathbf{C}$.

Our fundamental definition is:

8.2. Definition. $T(\cdot)$ is called a $W$-to-He, countably additive, quasiisometric (c.a.q.i.) measure over $(\Lambda, P, M)$, iff

(i) $M(\cdot)$ is a $W$-to- $W$, nonnegative, hermitian ${ }^{16}$ operator-valued measure on $P$, which is c.a. under the strong operator topology,

(ii) $\forall A \in P, T(A)$ is a continuous linear operator on $W$ to $\mathcal{H}$,

(iii) $\forall A, B \in \mathcal{P}, \quad T(B)^{*} T(A)=M(A \cap B)$.

We call $M(\cdot)$ the nonnegative hermitian measure of $T(\cdot)$.

An equivalent formulation of the condition 8.2(iii) is obviously

$$
\begin{aligned}
\forall A, B & \in \mathcal{P} \& \forall w, w^{\prime} \in W, \\
& \left(T(A)(w), T(B)\left(w^{\prime}\right)\right)_{\varkappa}=\left(M(A \cap B)(w), w^{\prime}\right)_{W} .
\end{aligned}
$$

In this the RHS could of course be written in the symmetric form

$$
\left(\sqrt{ } M(A \cap B)(w), \sqrt{ } M(A \cap B)\left(w^{\prime}\right)\right)_{W} .
$$

Thus we have

$$
\begin{array}{r}
|T(A)|_{B}^{2}=|M(A)|_{B}, \quad|T(A)(w)|_{\mathfrak{H}}^{2}=|\sqrt{ } M(A)(w)|_{W}, \\
A \in \mathcal{P}, w \in \mathcal{H},
\end{array}
$$

$|\cdot|_{B},|\cdot| x,|\cdot|_{W}$ being the Banach-norm, the norm in $\mathfrak{H C}$ and the norm in $W$. Hence $T(A)$ and $M(A)$ have the same null space in $W$, and of course $M(\cdot) \ll T(\cdot) \ll M(\cdot)$. But $T(\cdot)$ need not be an indefinite

16 I.e. continuous, linear and selfadjoint. 
integral of $M(\cdot)$, nor need its total variation measure $|T|(\cdot)$ be finite on sets in 8 . In case $M(\cdot)=\mu(\cdot) I$, where $I$ is the identity operator on $W$, the equation in 8.2 (iii) reduces to

$$
\left(T(A)(w), T(B)\left(w w^{\prime}\right)\right)_{\mathscr{F}}=\mu(A \cap B) \cdot\left(w, w^{\prime}\right)_{W},
$$

and we so recover the c.a.q.i. measures considered previously in $[28, \S 4],[31, \S 3]$.

The connection between c.a.q.i. and c.a.o.s. measures is easy to describe:

8.5. Triviality. Let (i) T be a W-to-HC, c.a.q.i. measure over $(\Lambda, \odot$, $M)$,

(ii) $\forall w, w^{\prime} \in W, \xi_{w}(\cdot)={ }_{d} T(\cdot)(w), \quad \mu_{w w^{\prime}}(\cdot)==_{d}\left(M(\cdot)(w), w^{\prime}\right)$.

Then $\forall w, w^{\prime} \in W$,

(a) $\xi_{w}(\cdot)$ is a $\mathfrak{F}$-valued, c.a.o.s. measure on $\mathbb{P}$ with nonnegative measure $\mu_{w w}(c f .[30,1.2])$;

(b) $\xi_{w}(\cdot), \xi_{w^{\prime}}(\cdot)$ are biorthogonal measures with cross-covariance measure $\mu_{w w^{\prime}}$, i.e.

$$
\forall A, B \in \mathcal{P}, \quad\left(\xi_{w}(A), \xi_{w^{\prime}}(B)\right)_{\Im \mathcal{C}}=\mu_{w w^{\prime}}(A \cap B) .
$$

Proof. The last equation is obvious. On setting $w^{\prime}=w$ and using our Equivalence Thm. [30, 1.8] we get (a).

The following lemma gives some properties of the measure $T(\cdot)$; in particular it explains our choice of the letter $W$ and justifies the appellation "c.a.q.i." used in 8.2:

8.6. Lemma. Let $T$ be a $W$-to-He, c.a.q.i. measure over $(\Lambda, \mathcal{P}, M)$. Then

(a) $W$ is a wandering space of $T$, i.e. cf. 1.3,

$$
A, B \in \mathcal{P} \& A \| B \Rightarrow T(A)(W) \perp T(B)(W) ;
$$

(b) ${ }^{17} \forall A \in \mathcal{P}$, cls. $\left[T(A)\{\sqrt{ } M(A)\}^{\sim 1}\right]$ is a partial isometry on $W$ to Je with $\mathscr{K}_{M(A)}$ as null space;

(c) $\forall A \in \odot$ such that $M(A)$ is invertible, $T(A)\{\sqrt{ } M(A)\}^{-1}$ is an isometry on $W$ to $\mathfrak{H}$;

(d) $\forall A, A_{1}, A_{2}, \cdots \in \odot \ni A_{k}$ are $\| \& \cup_{1}^{n} A_{k} \subseteq A$,

${ }^{17}$ For any closed (s.v.) linear operator $S$ from $W$ to $W$, the symbols $\mathfrak{D}_{S}, \mathfrak{T}_{S}, \mathfrak{R}_{S}$ will denote the domain, null-space, range of $S$. The symbol $S^{\sim 1}$ denotes the generalized inverse of $S$ [17] defined by $S^{\sim 1}={ }_{d} P_{\Re_{S}} \cdot S^{-1} \cdot P_{\mathrm{ols}} \cdot \Omega_{S}$, where $P_{\Re}$ is the orthogonal projection on $W$ onto the subspace $\mathfrak{T} . S^{\sim 1}$ is a s.v. closed, linear operator from $W$ to $W$ with the e.d. domain $\mathbb{R}_{S}+\mathbb{R}_{S} \frac{1}{3}$ and the range $D_{S} \cap \Re_{S} \frac{1}{\text {. }}$. 


$$
\left\{T(A)-\sum_{1}^{n} T\left(A_{k}\right)\right\}^{*}\left\{T(A)-\sum_{1}^{n} T\left(A_{k}\right)\right\}=M(A)-\sum_{1}^{n} M\left(A_{k}\right)
$$

(e) $T(\cdot)$ is c.a. strongly or under the Banach norm $|\cdot|_{B}$, according as $M(\cdot)$ is c.a. strongly or under $|\cdot|_{B}$.

Proof. (a) is obvious; for if $A \| B$, then $M(A \cap B)=0$ and hence by (8.3) $T(A)(w) \perp T(B)\left(w^{\prime}\right), \forall w, w^{\prime} \in W$.

(b) We recall first $[17,(5.1)]$ that for any (s.v.) selfadjoint linear operator $S$ from $W$ to $W$

$$
S S^{\sim 1} \subseteq \operatorname{cls} .\left(S S^{\sim 1}\right)=P_{\mathrm{ols}} . \Re_{S}=P_{\mathfrak{T}_{\mathrm{S}}} \text {. }
$$

Now let

(2) $A \in P, \quad S=\underset{d}{=} \sqrt{ } M(A), \quad R=T(A)\{\sqrt{ } M(A)\}^{\sim 1}=T(A) S^{\sim 1}$.

Then

$$
D_{R}=D_{S}^{\sim 1}=\Re_{S}+\Re_{S}^{\perp} \text { is e.d. in W. }
$$

Also, by (8.3), (2) and (1)

$$
\begin{aligned}
\forall w, w^{\prime} \in D_{R}, \quad\left(R(w), R\left(w^{\prime}\right)\right)_{\mathfrak{x}} & =\left(S S^{\sim 1}(w), S S^{\sim 1}\left(w^{\prime}\right)\right)_{W} \\
& =\left(J(w), J\left(w^{\prime}\right)\right)_{W},
\end{aligned}
$$

where

$$
J \underset{d}{=} P_{\mathrm{cls} . \mathscr{Q}_{S}}=P_{\Upsilon_{\mathrm{S}}}^{\perp}
$$

By (4) and (5) the restriction of $R$ to $R_{S}$ is an isometry and the restriction of $R$ to $R_{S}^{\frac{1}{S}}$ is zero, and so $R$ is a continuous linear operator. It follows by a routine argument that its closure $\bar{R}$ is a partial isometry on cls. $D_{R}$, i.e. by (3) on $W$, into $\mathcal{H C}$ and has $R_{S}^{\frac{1}{S}}$ as its null space. Since $\Re_{S}^{\frac{1}{S}}=\Re_{S}=\Re_{\sqrt{ } M(A)}=\Re_{M(A)}$, we have (b).

(c) follows immediately from (b).

(d) The desired equality emerges on expanding the LHS and employing 8.2(iii).

(e) Let $\forall k \geqq 1, A_{k} \in \odot, A_{k}$ be $\| \& A={ }_{d} \cup_{1}^{\infty} A_{k} \in \odot$. From (d) we see at once that

$$
\left|T(A)-\sum_{1}^{n} T\left(A_{k}\right)\right|_{B}^{2}=\left|M(A)-\sum_{1}^{n} M\left(A_{k}\right)\right|_{B},
$$

and 


$$
\begin{aligned}
\forall w \in W, \quad\left|\left\{T(A)-\sum_{1}^{n} T\left(A_{k}\right)\right\}(w)\right|_{x}^{2} & \\
= & \left(\left\{M(A)-\sum_{1}^{n} M\left(A_{k}\right)\right\}(w), w\right)_{W} .
\end{aligned}
$$

The two assertions in (e) clearly follow from these equalities.

The following lemma is useful.

8.7. Lemma. Let $T(\cdot)$ be a $W$-to-Je, c.a.q.i. measure over $\left(\Lambda, \wp_{0}, M\right)$, where $B_{0}$ is a ring over $\Lambda$. Then

(a) $\forall A, B \in \Re_{0} \& \forall w \in W$,

$$
\begin{aligned}
\{T(A)-T(B)\} *\{T(A)-T(B)\} & =M(A \Delta B) \\
|T(A)-T(B)|_{B}^{2} & =|M(A \triangle B)|_{B} \\
|T(A)(w)-T(B)(w)|_{\Re}^{2} & =|\sqrt{ } M(A \triangle B)(w)|_{W}^{2}
\end{aligned}
$$

(b) $\forall n \geqq 1 \& \forall A_{n}, A \in \bigotimes_{0}$,

$$
\operatorname{sim}_{n \rightarrow \infty} T\left(A_{n}\right)=T(A) \Leftrightarrow \operatorname{slim}_{n \rightarrow \infty} M\left(A_{n} \triangle A\right)=0 ;
$$

(c) the set $\beta_{0}$ is metrized by $\rho$, where

$$
\rho(A, B) \underset{d}{=}|M(A \triangle B)|_{B}, \quad A, B \in \bigotimes_{0}
$$

when sets $A, B$ for which $M(A \triangle B)=0$ are identified;

(d) with respect to the ||$_{B}$ norm lopology and hence also the strong operator topology, $T(\cdot)$ is uniformly continuous on the metric space $\Theta_{0}$. In particular $\forall n \geqq 1 \& \forall A_{n}, A \in B_{0}$,

$$
\rho\left(A_{n}, A\right) \rightarrow 0 \Rightarrow \lim _{n \rightarrow \infty}\left|T\left(A_{n}\right)-T(A)\right|_{B}=0 .
$$

Proof. (a) Expanding the LHS and using 8.2(iii) we get the first equation in (a), from which the second and third clearly follow on taking the appropriate norms.

(b) is clear, since from the third equation in (a)

$$
\begin{aligned}
\left|T\left(A_{n}\right)(w)-T(A)(w)\right|_{\mathfrak{r}}^{2} & =\left|\sqrt{ }\left(M\left(A_{n} \Delta A\right)(w)\right)\right|_{W}^{2} \\
& =\left(M\left(A_{n} \Delta A\right)(w), w\right)_{W} .
\end{aligned}
$$

(c) That $\rho$ is a metric is easily checked on noting that for $A \subseteq B$, we have $0 \leqq M(A) \leqq M(B)$ and therefore $|M(A)|_{B} \leqq|M(B)|_{B}$.

(d) From (a) and (c) we see that $\forall A, B \in \beta_{0} \& \forall w \in W$, 
1970]

$$
|T(A)-T(B)|_{B}^{2}=\rho(A, B)
$$

and

$$
|T(A)(w)-T(B)(w)|_{\Im C}^{2}=|w|^{2} \rho(A, B),
$$

whence (d) clearly follows.

8.8. Definition. Let $T$ be a $W$-to-Je, c.a.q.i. measure over $(\Lambda, \odot, M)$.

(a) We define the subspace of $T$ by

$$
S_{T}=\mathfrak{d}\{T(A)(W): A \in \mathcal{S}\} \subseteq \mathfrak{H} .
$$

(b) We call $T$ He-basic iff $\mathrm{S}_{T}=\mathfrak{H}$.

From 8.8 it follows at once that

$$
S_{T}=\text { cls. } \sum_{A \in \mathcal{P}} \Re_{T(A)} \quad \& \quad \stackrel{\perp}{S_{T}}=\bigcap_{A \in \mathcal{P}} \Re_{T(A) *} ;
$$

and if $P_{S_{T}}$ is the projection on $\mathcal{H C}$ onto $S_{T}$, then

$$
\forall A \in \odot, \quad T(A)^{*}=T(A)^{*} P s_{T_{r}}
$$

Also

(8.11) $\forall x \in \mathcal{H}, \quad \zeta_{x}(\cdot)=T(\cdot)^{*}(x)$ is a $W$-valued, c.a. measure on $\mathrm{P}$.

In general $\zeta_{x}(\cdot)$ will not be orthogonally scattered.

As with c.a.o.s. measures we need to consider the case $\Lambda \subseteq R$. In this case we again find a natural correspondence between operatorvalued point-functions and set-functions governed by the analogues of 2.12 and 2.13. However, the following result enunciated in terms of set-functions alone will suffice for our purpose:

8.12. LemMA. Let

(i) $\odot$ be the prering of intervals $(a, b] \ni 0 \leqq a<b<\infty$,

(ii) $\forall J \in \mathcal{P}, T(J)$ be a continuous, linear operator on $W$ to $\mathfrak{H}$,

(iii) $T(\cdot)$ be a finitely-additive measure on $P$,

(iv) $\exists$ a function $F$ on $[0, \infty)$ such that

$$
T(0, t]^{*} T(0, s]=F(\min \{s, t\}), \quad s, t \geqq 0 .
$$

Then

(a) $F$ is a monotone increasing, W-to- $W$, nonnegative hermitian operator-valued function on $[0, \infty)$;

(b) $T(\cdot)$ is a W-to-JC, c.a.q.i. measure over $([0, \infty), \odot, M)$, where $M$ 
is the $W$-to- $W$, nonnegative, hermitian operator-valued measure on $\mathrm{\rho}$ induced by $F$.

Proof. (a) By (iv)

$$
\forall t \geqq 0, \quad F(t)=T(0, t]^{*} T(0, t],
$$

and this shows that $F(t)$ is a $W$-to- $W$, nonnegative, hermitian operator. Next, by (iii) $\forall a, b, c, d \geqq 0$,

$$
\begin{aligned}
T(c, d]^{*} T(a, b]= & \left\{T(0, d]^{*}-T(0, c]^{*}\right\}\{T(0, b]-T(0, a]\} \\
= & F(\min \{b, d\})-F(\min \{a, d\})-F(\min \{b, c\}) \\
& +F(\min \{a, c\}) .
\end{aligned}
$$

For $a<b \leqq c<d$, this yields $T(c, d]^{*} T(a, b]=0$, and adjoining, $T(a, b]^{*} T(c, d]=0$. This shows that

$$
A, B \in \mathcal{P} \& A \| B \Rightarrow T(B) * T(A)=0 .
$$

Consequently, for $0 \leqq a<b$,

$$
\begin{array}{rlr}
F(b) & =T(0, b]^{*} T(0, b] & \text { by (1) } \\
& =\left\{T(0, a]^{*}+T(a, b]^{*}\right\}\{T(0, a]+T(a, b]\}, & \text { by (iii) } \\
& =F(a)+T(a, b]^{*} T(a, b] \geqq F(a) & \text { by (1) \& (3). }
\end{array}
$$

This completes the proof of (a).

(b) Let $A, B \in \mathcal{P}$; for definiteness let $A=(a, b], B=(c, d]$ where $a \leqq c \leqq b \leqq d$. Then by (2)

$$
\begin{aligned}
T(B)^{*} T(A) & =F(b)-F(a)-F(c)+F(a) \\
& =F(b)-F(c)=M(b, c]=M(A \cap B) .
\end{aligned}
$$

We verify (4) similarly for other orderings of $a, b, c, d$. By (ii) and (4) we have (b).

\section{On the Hilbert space $L_{2}(\Lambda, ß, M ; W)$}

In this section

(i) $W$ is a separable Hilbert space over $\mathbf{C}$;

(ii) $B$ is a $\sigma$-ring over an arbitrary set $\Lambda$;

(iii) $M(\cdot)$ is a $W$-to- $W$, nonnegative, hermitian operator-valued measure on a sub $\delta$-ring $\beta_{0}$ of $B, c . a$. under the strong operator topology.

Our primary purpose is to comment on the nature of the space

$$
\mathfrak{L}_{2, W}=L_{2}(\Lambda, \Theta, M ; W)
$$


of $W$-valued, B-measurable functions on $\Lambda$, which are "square integrable" with respect to the operator-valued measure $M(\cdot)$. But with an eye to future developments (cf., footnote 15), in the first part, A, of this section we shall first consider the more general space

$$
\mathfrak{L}_{2}=\mathfrak{L}_{2, W^{\prime \circ W}}=L_{2}\left(\Lambda, \leftrightarrow, M ; W^{\prime} \circ W\right)
$$

where $W^{\prime}$ is a finite-dimensional ${ }^{18}$ Hilbert space over $\mathbf{C}$ and $W^{\prime} \circ W$ is the tensor product of $W^{\prime}$ and $W$. Then in part $\mathrm{B}$ we shall revert to $\mathfrak{L}_{2, W}$ by taking $W^{\prime}=\mathrm{C}$ and exploiting the natural isomorphism between the Hilbert spaces CoW and $W$.

A. The tensor product $W^{\prime} \circ W$ is by definition the set of all Hilbert-Schmidt operators on $W^{\prime}$ to $W$. It is a Hilbert space under the Euclidean or Hilbert-Schmidt inner product and norm:

$$
\begin{aligned}
\forall S, T \in W^{\prime} \circ W, \quad(T, S)_{E}=\operatorname{trace} S^{*} T, \\
|T|_{E}=\sqrt{ }(T, T)_{E} .
\end{aligned}
$$

The trace is well defined and finite, since $S$ and $T$ are HilbertSchmidt. Moreover, since $\operatorname{dim} . W^{\prime}<\infty, W^{\prime} \circ W^{\prime}$ is homeomorphic to the Banach space $\mathrm{CL}\left(W^{\prime}, W^{\prime}\right)$ of continuous, linear operators on $W^{\prime}$ to $W^{\prime}$ under the Banach-norm.

To define integration with respect to $M$ we have to consider an even more general set-up. ${ }^{19}$ Let $\Phi$ be a function on $\Lambda$ to $W^{\prime} \circ W$ and $\Psi$ a function on $\Lambda$ to $W \circ W^{\prime \prime}$, where $W^{\prime \prime}$ is a (third) Hilbert space. Then $\forall \lambda_{j} \in \Lambda \& \forall \Delta_{j} \in \Theta_{0}$

$$
\sum_{1}^{r} \Psi\left(\lambda_{j}\right) M\left(\Delta_{j}\right) \Phi\left(\lambda_{j}\right) \in W^{\prime} \circ W^{\prime \prime} .
$$

This suggests the possibility of defining

$$
\int_{\Lambda} \Psi(\lambda) M(d \lambda) \Phi(\lambda)
$$

as a member of $\mathrm{CL}\left(W^{\prime}, W^{\prime \prime}\right)$ in a reasonable way, so that the resulting theory is a consistent extension of the theories known for special cases such as $W^{\prime \prime}=W^{\prime}=\mathrm{C}, M(\cdot)=\mu(\cdot) I_{W}, \mu(\cdot)$ being a nonnegative measure, and $I_{W}$ the identity operator on $W$. To fulfill this requirement the integrals (3) have to be bilinear, i.e.

${ }^{18}$ We assume finite-dimensionality only for reasons of simplicity. Actually we could consider any separable Hilbert space $W^{\prime}$.

${ }^{19}$ It is these facts which account for the length of the section, even though it is only ancillary to our main subject. 


$$
\begin{aligned}
& \text { (a) for integrable } \Phi_{i} \& \Psi_{i} \text { on } \Lambda \text { to } W^{\prime} \circ W \& W \circ W^{\prime \prime} \text {, } \\
& \int_{\Lambda}\left\{\Psi_{1}(\lambda)+\Psi_{2}(\lambda)\right\} M(d \lambda)\left\{\Phi_{1}(\lambda)+\Phi_{2}(\lambda)\right\} \\
& =\sum_{i=1}^{2} \sum_{j=1}^{2} \int_{\Lambda} \Psi_{i}(\lambda) M(d \lambda) \Phi_{j}(\lambda), \\
& \ni A \cap B \in \Theta_{0} \text {, } \\
& \int_{\Lambda} S \cdot \chi_{A}(\lambda) M(d \lambda) R \cdot \chi_{B}(\lambda)=S \cdot M(A \cap B) \cdot R .
\end{aligned}
$$

For simple functions, we will then get the expected result:

$$
\forall R_{j} \in W^{\prime} \circ W, \quad \forall S_{k} \in W \circ W^{\prime \prime} \& \forall A_{j}, B_{k} \in \mathbb{B}^{\text {loc }} \ni A_{j} \cap B_{k} \in \bigotimes_{0}
$$

$$
\begin{aligned}
\int_{\Lambda}\left\{\sum_{1}^{s} S_{k} \chi_{B_{k}}(\lambda)\right\} M(d \lambda)\left\{\sum_{1}^{r} R_{j} \chi_{A_{j}}(\lambda)\right\} & \\
& =\sum_{j=1}^{r} \sum_{k=1}^{s} S_{k} M\left(A_{j} \cap B_{k}\right) R_{j} .
\end{aligned}
$$

Now suppose that the integrals (3) have been defined so as to satisfy (9.3) and other similar requirements we might impose. Then it would be natural to define the space $\mathscr{L}_{2}$ of $(2)$ by

$$
\mathfrak{L}_{2}=\left\{\Phi: \Phi_{d}^{*} \text { is on } \Lambda \text { to } W^{\prime} \circ W \& \int_{\Lambda} \Phi(\lambda)^{*} M(d \lambda) \Phi(\lambda) \text { exists }\right\} .
$$

Since the adjoint $\Phi(\lambda) *$ of $\Phi(\lambda)$ is in $W \circ W^{\prime}$, the last integral is in $\mathrm{CL}\left(W^{\prime}, W^{\prime}\right)$; thus we are taking $W^{\prime \prime}=W^{\prime}$. Now a crucial property of $\mathfrak{L}_{2}$ in the classical case, viz. $W^{\prime}=W=\mathbf{C} \& M=\mu$, is its Hilbert spacedness under the inner product

$$
(\Phi, \Psi)_{\mu}=\int_{\Lambda} \Phi(\lambda) \Psi(\lambda) \mu(d \lambda),
$$

and the everywhere-denseness of the $C$-valued, $B_{0}$-simple functions in it. For higher dimensional $W^{\prime}, W$ the natural analogue of this inner product is

$$
(\Phi, \Psi)_{M}=\operatorname{trace} \int_{\Delta} \Psi(d \lambda) * M(d \lambda) \Phi(\lambda)
$$

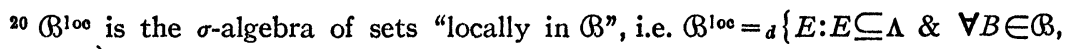
$E \cap B \in \mathbb{B}\}$. 
the trace being finite since the integral is in $\mathrm{CL}\left(W^{\prime}, W^{\prime}\right)$ and $W^{\prime}$ is finite dimensional. A measure $M$ would be somewhat unsatisfactory for our purposes, were $\mathfrak{L}_{2}$ to be incomplete under the norm induced by this inner product, viz.

$$
|\Phi|_{M} \underset{d}{=} \sqrt{ }(\Phi, \Phi)_{M}=\sqrt{ } \operatorname{trace} \int_{\Lambda} \Phi(\lambda) * M(d \lambda) \Phi(\lambda) .
$$

It would also be inadequate, were the linear manifold of $W^{\prime} \circ W$ valued, $\wp_{0}$-simple functions:

$$
\varsigma\left(\Lambda, B_{0} ; W^{\prime} \circ W\right)
$$

$$
=\left\{\sum_{1}^{r} R_{k} \chi_{A_{k}}: r \geqq 1, R_{k} \in W^{\prime} \circ W \& A_{k} \in B_{0}\right\}
$$

not dense in $\mathfrak{L}_{2}$. We are thus led to the following criterion for the adequacy of the measure $M$ :

9.9. Criterion. A measure $M$ satisfying (9.1)(iii) is adequate, iff it allows us to define the integrals (3) so as to ensure (9.3), etc., and to make the $\mathfrak{L}_{2}$ of (9.5) a Hilbert space under the inner product (9.6), having $\varsigma\left(\Lambda, \Theta_{0} ; W^{\prime} \circ W\right)$ as an e.d. linear manifold, when functions $\Phi, \Psi$ such that $|\Phi-\Psi|_{M}=0$ are identified.

We shall now describe two known types of adequate measures.

Let the measure $M$ of (9.1)(iii) be the indefinite integral of a nonnegative measure; i.e. suppose that

$\exists$ a nonnegative, $\sigma$-finite, c.a. measure $\mu$ on $@$ for which $\beta_{\mu}=\bigotimes_{0}$, and $\exists$ a function ${ }^{21}$

$$
M_{\mu}^{\prime}(\cdot) \in L_{1}^{100}(\Lambda, \AA, \mu ; \mathrm{CL}(W, W))
$$

(9.10) such that

$$
\forall B \in B_{\mu}, \quad M(B)=\int_{B} M_{\mu}^{\prime}(\lambda) \mu(d \lambda)=\int_{\Lambda} M_{\mu}^{\prime}(\lambda) \chi_{B}(\lambda) \mu(d \lambda),
$$

the last being a Bochner integral.

From the properties of the Bochner integral it follows that

$$
\begin{gathered}
\forall w, w^{\prime} \in W \& \forall B \in B_{\mu}, \\
\left(M(B) w, w^{\prime}\right)_{W}=\int_{B}\left(M_{\mu}^{\prime}(\lambda) w, w^{\prime}\right)_{W} \mu(d \lambda),
\end{gathered}
$$

${ }^{21}$ For any Banach space $X$, we say that $F \in L_{1}^{\text {loo }}(\Lambda, Q, \mu ; X)$, iff $F$ is on $\Lambda$ to $X$ and $\forall B \in B_{\mu}, F(\cdot) \chi_{B}(\cdot)$ is Bochner integrable on $\Lambda$ to $X$. $\beta_{\mu}$ is as in 2.1(iii). 
i.e. the measure $\mu_{w w^{\prime}}$ of (8.5) (ii) is absolutely continuous with respect to $\mu$ and
$\left(9.11^{\prime}\right)$
$d \mu_{w w^{\prime}} / d \mu=\left(M_{\mu}^{\prime}(\cdot) w, w^{\prime}\right)_{W}$,
a.e. $(\mu)$.

For measures subject to $(9.10)$ it is natural to define the integrals (3) by

$$
\int_{\Lambda} \Psi(\lambda) M(d \lambda) \Phi(\lambda)=\int_{\Lambda} \Psi(\lambda) M_{\mu}^{\prime}(\lambda) \Phi(\lambda) \mu(d \lambda),
$$

the last being a Bochner integral. Then by (9.5)

$$
\begin{aligned}
\Phi \in \mathfrak{L}_{2} \Leftrightarrow & \Phi \text { is on } \Lambda \text { to } W^{\prime} \circ W, \quad \text { \& } \\
& \Phi(\cdot)^{*} M_{\mu}^{\prime}(\cdot) \Phi(\cdot) \in L_{1}\left(\Lambda, \Theta, \mu ; W^{\prime} \circ W^{\prime}\right) .
\end{aligned}
$$

It is known that

for $M$ as in (9.10), $\mathfrak{L}_{2}$ defined by (9.13) is a pre-Hilbert space under

the inner product $(,)_{M}$ when functions $\Phi, \Psi \ni|\Phi-\Psi|_{M}=0$ are identified.

Since $W^{\prime}$ is finite dimensional, this follows for instance from [26, 4.7]. But as shown in [21, p. 71], even with $W^{\prime}=C, \mathfrak{L}_{2}$ need not be complete. A further restriction on $M$ sufficient to ensure the completeness of $\mathfrak{L}_{2}$ and the denseness of $S\left(\Lambda, \mathbb{B}_{\mu} ; W^{\prime} \circ W\right)$ in $\mathfrak{L}_{2}$ is that

$$
\max _{B \in B_{\mu}} \operatorname{rank} M(B)<\infty \text {. }
$$

This is a simple extension of a result of Rosenberg $[44,3.9,3.11]$. To sum up, a measure $M$ satisfying (9.1)(iii), (9.10) and (9.15) is adequate according to Criterion 9.9; for such measures, the integrals (3) are defined by (9.12).

Another kind of measure, which we shall encounter is bounded and "diagonal" in the sense that

the $B \& B_{0}$ of (9.1) are identical $\sigma$-algebras;

$$
\forall B \in B_{0}, \quad M(B)=\sum_{j \in J} \mu_{j}(B) P_{j},
$$

where $J$ is a (finite or infinite) set of positive integers, and the series converges strongly when $J$ is infinite, $\mu_{j}$ are c.a. probability measures on B such that

$$
j, k \in J \& k>j \Rightarrow \mu_{k} \ll \mu_{j},
$$

$P_{j}$ are orthogonal projections of rank 1 on $W$ to $W, \ni$

$$
\sum_{j \in J} P_{j}=I_{W}, \quad P_{i} P_{j}=0, \quad i, j \in J, \quad i \neq j .
$$


For such measures the integrals (3) have to be defined by

$$
\int_{\Lambda} \Psi(\lambda) M(d \lambda) \Phi(\lambda)=\sum_{d \in J} \int_{\Lambda} \Psi(\lambda) P_{j} \Phi(\lambda) \mu_{j}(d \lambda),
$$

it being presumed that the pair $(\Phi, \Psi)$ is such that

$$
\forall j \in J, \quad \Psi(\cdot) P_{j} \Phi(\cdot) \in L_{1}\left(\Lambda, \propto, \mu_{j} ; W^{\prime} \circ W^{\prime \prime}\right),
$$

and that for infinite $J$, the series in (9.17) converges strongly. It follows from (9.5) that

$$
\Phi \in \mathfrak{L}_{2} \text {, iff } \Phi \text { is on } \Lambda \text { to } W^{\prime} \circ W,
$$

$$
\begin{aligned}
& \forall j \in J, \quad P_{j} \Phi(\cdot) \in L_{2}\left(\Lambda, \mathbb{B}, \mu_{j} ; W^{\prime} \circ W\right), \quad \& \\
& \sum_{j \in J} \int_{\Lambda} \Phi(\lambda)^{*} P_{j} \Phi(\lambda) \mu_{j}(d \lambda) \text { cgs. strongly. }
\end{aligned}
$$

The strong convergence is equivalent to convergence under the norms ||$_{B}$ or ||$_{E}$, cf. (9.2), since the inregrals are in $\mathrm{CL}\left(W^{\prime}, W^{\prime}\right)$ and dim. $W^{\prime}<\infty$. From (9.6) and (9.7) we now get

$$
\begin{aligned}
\forall \Phi, \Psi \in \mathcal{L}_{2}, \quad(\Phi, \Psi)_{M} & =\sum_{j \in J} \int_{\Lambda}\left(P_{j} \cdot \Phi(\lambda), P_{j} \cdot \Psi(\lambda)\right)_{E} \mu_{j}(d \lambda) \\
|\Phi|_{M}^{2} & =\sum_{j \in J} \int_{\Lambda}\left|P_{j} \cdot \Phi(\lambda)\right|_{E}^{2} \mu_{j}(d \lambda) .
\end{aligned}
$$

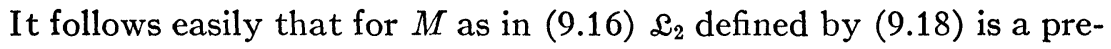
Hilbert space under the inner product ( , $)_{M}$ of (9.19) when functions $\Phi$, $\Psi$ such that $|\Phi-\Psi|_{M}=0$ are identified. But we have not been able to establish its completeness. We can show, however, that if the $\mu_{j}$ in (9.16) are related by the equalities:

$$
\forall j \in J, \quad \mu_{j}(\cdot)=\mu\left(\Lambda_{j} \cap \cdot\right) / a_{j}
$$

where $\mu$ is a c.a. probability measure on $B$

$$
\begin{gathered}
\Lambda_{j} \in \Theta \quad \& \quad \Lambda_{k} \subseteq \Lambda_{j} \subseteq \Lambda_{1}=\Lambda, \quad \forall j, k \in J, k>j \\
\& \quad a_{j}=\mu\left(\Lambda_{j}\right)>0,
\end{gathered}
$$

the resulting $\mathscr{L}_{2}$ is complete and $\delta\left(\Lambda, \bigotimes_{0} ; W^{\prime} \circ W\right)$ is e.d. in it. ${ }^{22}$ To sum up, a measure $M$ satisfying (9.1)(iii), (9.16) and (9.20) is adequate according to Criterion 9.9; for such measures the integrals (3) are defined by (9.17).

${ }^{22}$ The proof will appear in the printed version of [26, Appendix] by Mandrekar and Salehi, to whom the writer is grateful for discussion and criticism. 
B. We shall now bring these general results on $\mathfrak{L}_{2, W}$ oW to bear on the space $\mathfrak{L}_{2, W}$ of (1) by treating it as $\mathfrak{L}_{2}$, Cow , i.e. by taking $W^{\prime}=C$. To each $w \in W$ corresponds $S_{w} \in C \circ W$ defined by

$$
S_{w}(z)=z w \in W, \quad \forall z \in C .
$$

It follows at once that

(4) $\forall w, w^{\prime} \in W, \quad w=S_{w}(1), \quad \& \quad S_{w}^{*}\left(w^{\prime}\right)=\left(w^{\prime}, w\right)_{W}=\left(S_{w}^{*} \cdot S_{w^{\prime}}\right)(1)$. Obviously, the correspondence: $w \rightarrow S_{w}$ is a unitary operator on the Hilbert space $W$ onto the Hilbert space $\mathrm{C} \circ W$. To dispense ultimately with the cumbersome $S_{w}$ notation, we shall stretch our vector notation by writing:

$$
\begin{aligned}
& \forall w_{1}, w_{2} \in W, \quad \& \quad \forall N \in \mathrm{CL}(W, W), \\
& w_{2}^{*} N w_{1}=\left(N\left(w_{1}\right), w_{2}\right)_{W}=\left(S_{w_{2}}^{*} N S_{w_{1}}\right)(1),
\end{aligned}
$$

where the last equality is clear from (4).

Now let $\phi, \psi$ be functions on $\Lambda$ to $W$. Then $S_{\phi(\cdot)}, S_{\psi(\cdot)}$ are functions on $\Lambda$ to $C \circ W$, and by (9.22) $\forall \lambda_{k} \in \Lambda \& \forall \Delta_{k} \in \beta_{0}$,

$$
\begin{aligned}
\sum_{1}^{r} \psi\left(\lambda_{k}\right)^{*} M\left(\Delta_{k}\right) \phi\left(\lambda_{k}\right) & =\sum_{1}^{r}\left(M\left(\Delta_{k}\right) \phi\left(\lambda_{k}\right), \psi\left(\lambda_{k}\right)\right)_{W} \\
& =\left\{\sum_{1}^{r} S_{\psi\left(\lambda_{k}\right)}^{*} \cdot M\left(\Delta_{k}\right) \cdot S_{\phi\left(\lambda_{k}\right)}\right\}(1) .
\end{aligned}
$$

This equation suggests the following definition:

9.23. Definition. For $\phi, \psi$ on $\Lambda$ to $W$, the integral $\int_{\Lambda} \psi^{*}(\lambda) M(d \lambda) \phi(\lambda)$ is said to exist iff $\int_{\Lambda} S_{\psi(\lambda)}^{*} M(d \lambda) S_{\phi(\lambda)}$ exists; furthermore

$$
\int_{\Lambda} \psi(\lambda)^{*} M(d \lambda) \phi(\lambda)=\left\{\int_{\Lambda} S_{\psi(\lambda)}^{*} M(d \lambda) S_{\phi(\lambda)}\right\}(1) .
$$

It follows from (9.5) that

$\&$

$$
\phi \in \mathfrak{L}_{2, W} \Leftrightarrow S_{\phi(\cdot)} \in \mathfrak{L}_{2, \mathrm{Co} W}
$$

$$
\begin{aligned}
\forall \phi, \psi \in \mathcal{L}_{2, W}, \quad(\phi, \psi)_{M} & =\int_{d} \psi(\lambda)^{*} M(d \lambda) \phi(\lambda) \\
& |\phi|_{M} \underset{d}{=} \sqrt{ }(\phi, \phi)_{M}=\int_{\Lambda} \phi(\lambda)^{*} M(d \lambda) \phi(\lambda) .
\end{aligned}
$$


In the following theorems and corollaries we list some properties of functions in $\mathfrak{L}_{2, W}$ and of their integrals:

9.25. TheOREM. Let

(i) the measure $M$ be as in (9.1)(iii),

(ii) $\forall w, w^{\prime} \in W \& \forall B \in \beta_{0}, \mu_{w w^{\prime}}(B)={ }_{d}\left(M(B) w, w^{\prime}\right)_{W}, c f$. (8.5)(iii),

(iii) $\forall E \in B^{\mathrm{loc}}, \int_{E} \psi(\lambda)^{*} M(d \lambda) \phi(\lambda)={ }_{d} \int_{\Lambda} \chi_{E}(\lambda) \psi(\lambda) * M(d \lambda) \phi(\lambda)$,

(iv) $\forall E \in B^{\text {loc }}$

$$
\mathfrak{L}_{2, W}(E)=\left\{\phi: \phi \in \mathfrak{L}_{2, W} \quad \& \quad \phi \text { vanishes, a.e. }(M), \text { on } \Lambda \backslash E\right\} .
$$

Then

(a) when $M$ is adequate, $c f .9 .9, \mathfrak{L}_{2, w}$ is a Hilbert space and the linear manifold

$$
\mathcal{S}\left(\Lambda, \Theta_{0} ; W\right)=\left\{\sum_{1}^{r} w_{k} \chi_{A_{k}}: \quad r \geqq 1, \quad w_{k} \in W \quad \& \quad A_{k} \in \bigotimes_{0}\right\}
$$

of $ß_{0}$-simple functions on $\Lambda$ to $W$ is e.d. in $\mathfrak{L}_{2, W}$;

(b) $\forall w_{j}, w_{k}^{\prime} \in W \& \forall A_{j}, B_{k} \in \bigotimes_{0}$,

$$
\begin{aligned}
\int_{\Lambda}\left\{\sum_{k=1}^{s} w_{k}^{\prime} \chi_{B_{k}}(\lambda)\right\}^{*} M(d \lambda)\left\{\sum_{j=1}^{r} w_{j} \chi_{A_{j}}(\lambda)\right\} & \\
& =\sum_{j=1}^{r} \sum_{k=1}^{s}\left(M\left(A_{j} \cap B_{k}\right) w_{j}, \quad w_{k}\right)_{W} ;
\end{aligned}
$$

(c) $\forall B \in \mathbb{B}^{\mathrm{loc}}, \forall A_{j} \in \mathbb{B}_{0} \& \forall w_{j} \in W$

$$
\int_{B} M(d \lambda)\left\{\sum_{j=1}^{r} w_{j} \chi_{A_{j}}(\lambda)\right\}=\sum_{j=1}^{r} M\left(B \cap A_{j}\right)\left(w_{j}\right) ;
$$

(d) $\forall \phi_{1}, \phi_{2} \in \mathcal{L}_{2, W},\left(\phi_{1}, \phi_{2}\right)_{M}=\left(\phi_{1} \chi_{S_{1}}, \phi_{2} \chi_{S_{2}}\right)_{M}$, where $S_{1}, S_{2}$ are the supports of $\phi_{1}, \phi_{2}$;

(e) $\forall n \geqq 1, E_{n} \in \mathbb{B}^{\text {loc }}, E_{n}$ are $\| \& \cup_{1}^{\infty} E_{n}=E$

$$
\Rightarrow \quad \mathfrak{L}_{2, W}(E)=\sum_{n=1}^{\infty} \mathfrak{L}_{2, W}\left(E_{n}\right) \quad \& \quad \mathfrak{L}_{2, W}\left(E_{m}\right) \perp \mathfrak{L}_{2, W}\left(E_{n}\right) ;
$$

(f) $\forall n \geqq 1, E_{n} \in B^{\text {loc }}, E_{n}$ are $\|, \cup_{1}^{\infty} E_{n}=E, \& \phi, \psi \in \mathfrak{L}_{2, W}(E) \Rightarrow$

$$
\begin{aligned}
& \forall n \geqq 1, \quad \phi, \psi \in \mathscr{L}_{2, W}\left(E_{n}\right), \quad \text { and } \\
& \sum_{n=1}^{\infty} \int_{E_{n}} \psi(\lambda)^{*} M(d \lambda) \phi(\lambda) c g s . \&=\int_{E} \psi(\lambda)^{*} M(d \lambda) \phi(\lambda) ;
\end{aligned}
$$


(g) $\phi_{n} \rightarrow \phi$ in $\mathfrak{L}_{2, W}$, i.e. $\left|\phi_{n}-\phi\right|_{M} \rightarrow 0$,

$$
\Rightarrow \forall B \in \mathbb{B}^{\mathrm{loc}},\left|\int_{B} M(d \lambda) \phi_{n}(\lambda)-\int_{B} M(d \lambda) \phi(\lambda)\right|_{W} \rightarrow 0 ;
$$

(h) $\forall \phi$ on $\Lambda$ to $C, \quad w \phi(\cdot) \in \mathscr{L}_{2, W} \Leftrightarrow \phi \in L_{2}\left(\Lambda, ®, \mu_{w w} ; C\right)$;

(j) $\forall \phi, \psi$ on $\Lambda$ to $C$,

$$
w \phi(\cdot), w^{\prime} \psi(\cdot) \in \mathfrak{L}_{2, W} \Rightarrow \phi(\cdot) \Psi(\cdot) \in L_{1}\left(\Lambda, \leftrightarrow, \mu_{w, w^{\prime}} ; C\right) ;
$$

(k) $\forall w \phi(\cdot), w^{\prime} \psi(\cdot) \in \mathfrak{L}_{2, W}$,

$$
\begin{aligned}
\left(w \phi(\cdot), w^{\prime} \psi(\cdot)\right)_{M} & =\int_{\Lambda} \phi(\lambda) \overline{\psi(\lambda)} \mu_{w w^{\prime}}(d \lambda) \\
|w \phi(\cdot)|_{M}^{2} & =\int_{\Lambda}|\phi(\lambda)|^{2} \mu_{w w}(d \lambda) .
\end{aligned}
$$

9.26. THEOREM. Let

(i) the measure $M$ be as in 9.1(iii),

(ii) $\phi$ be a B-measurable function on $\Lambda$ to $W$,

(iii) $\Theta_{\phi}={ }_{d}\left\{B: B \in B^{\text {loo }} \& \phi \in \mathscr{L}_{2, W}(B)\right\}, c f$. 9.25(iv),

(iv) $\forall B \in \Re_{\phi}, \quad \nu_{\phi \phi}(B)=_{d} \int_{B} \phi(\lambda) * M(d \lambda) \phi(\lambda)$.

\section{Then}

(a) $B_{\phi}$ is a $\delta$-subring of $\otimes^{\text {loc }}$, and $\nu_{\phi \phi}$ is a finite (possibly unbounded) nonnegative, c.a. measure on $B_{\phi}$;

(b) $\Theta_{\phi}=\Theta_{\tilde{\nu}}={ }_{\boldsymbol{d}}\left\{B: B \in \sigma\left(\Theta_{\phi}\right), \& \tilde{\nu}(B)<\infty\right\}$, where $\tilde{\nu}$ is the Hahn extension of $\nu_{\phi \phi}$ to the $\sigma$-ring, $\sigma\left(\Theta_{\phi}\right)$, generated by $\beta_{\phi}$;

(c) $\forall f \in L_{2}\left(\Lambda, \sigma\left(\mathbb{B}_{\phi}\right), \tilde{\nu} ; C\right), \quad f(\cdot) \phi(\cdot) \in \mathfrak{L}_{2, W}$ and

$$
\int_{\Lambda}\{f(\lambda) \phi(\lambda)\}^{*} M(d \lambda)\{f(\lambda) \phi(\lambda)\}=\int_{\Lambda}|f(\lambda)|{ }^{2} \nu_{\phi \phi}(d \lambda) .
$$

9.27. THEOREM. Let

(i) the measure $M$ be as in (9.1)(iii)

(ii) $S$ be a continuous linear operator on a Hilbert space $W^{\prime}$ to $W$,

(iii) $\forall B \in \Theta_{0}, \quad N(B)={ }_{d} S^{*} M(B) S$.

\section{Then}

(a) $N$ is a $W^{\prime}$-to- $W^{\prime}$, nonnegative, hermitian, operator-valued measure on $B_{0}$, c.a. under the strong operator-topology;

(b) $\phi \in \mathscr{L}_{2}\left(\Lambda, \Theta, N ; W^{\prime}\right) \Rightarrow S\{\phi(\cdot)\} \in \mathscr{L}_{2}(\Lambda, \Theta, M ; W)$;

(c) $\forall \phi, \psi \in \mathfrak{L}_{2}\left(\Lambda, \AA, N ; W^{\prime}\right)$

$$
\int_{\Delta} \psi(\lambda)^{*} N(d \lambda) \phi(\lambda)=\int_{\Lambda}\{S \psi(\lambda)\}^{*} M(d \lambda)\{S \phi(\lambda)\} .
$$


9.28. Corollary. Let the measures $M$ and $\mu$ satisfy (9.10) and (9.15). Then

(a) $\phi \in L_{2, W} \Leftrightarrow\left\{\sqrt{ } M_{\mu}^{\prime}(\cdot)\right\} \phi(\cdot) \in L_{2}(\Lambda, \Re, \mu ; W)$;

(b) $\forall \phi, \psi \in \mathfrak{L}_{2, W},(\phi, \psi)_{M}=\int_{\Lambda}\left(M_{\mu}^{\prime}(\lambda) \phi(\lambda), \psi(\lambda)\right)_{W \mu}(d \lambda)$

$$
|\phi|_{M}^{2}=\int_{\Lambda}\left|\sqrt{ }\left\{M_{\mu}^{\prime}(\lambda)\right\} \phi(\lambda)\right|_{W \mu}^{2}(d \lambda)
$$

(c) $\forall \phi \in \mathfrak{L}_{2, W} \& \forall B \in \Re_{\mu}, \int_{B} M(d \lambda) \phi(\lambda)=\int_{B} M_{\mu}^{\prime}(\lambda) \phi(\lambda) \mu(d \lambda)$;

(d) $\forall \phi \in \mathfrak{L}_{2, W}, \quad \phi(\cdot)=J_{\mu}(\cdot)\{\phi(\cdot)\}$, where $J_{\mu}(\lambda)$ is the projection on $W$ onto $\Re_{\bar{M}_{\mu}{ }^{\prime}(\lambda)}^{\frac{1}{}}$.

The next result is obvious from (9.18), (9.19) and (9.24) despite the inadequacy of $M$ :

9.29. Triviality. Let the measure $M$ of (9.1)(iii) satisfy (9.16). Then

(a) $\phi \in \mathcal{L}_{2, W}$, iff $\phi$ is on $\Lambda$ to $W$,

$$
\begin{array}{r}
\forall j \in J, P_{j} \phi(\cdot) \in L_{2}\left(\Lambda, 囚, \mu_{j} ; W\right) \\
\& \sum_{j \in J} \int_{\Lambda}\left|P_{j} \phi(\lambda)\right|_{W}^{2} \mu_{j}(d \lambda)<\infty ;
\end{array}
$$

(b) $\forall \phi, \psi \in \mathfrak{L}_{2, W},(\phi, \psi)_{M}=\sum_{j \in J} \int_{\Lambda}\left(P_{j} \phi(\lambda), \psi(\lambda)\right)_{W} \mu_{j}(d \lambda)$

$$
|\phi|_{M}^{2}=\sum_{j \in J} \int_{\Lambda}\left|P_{j} \phi(\lambda)\right|_{W}^{2} \mu_{j}(d \lambda) \text {. }
$$

Finally, we must record for Besicovitch spaces the following theorem on the differentiation of Bochner integrals:

9.30. Differentiation theorem. Let

(i) $(\Lambda, \beta)$ be a Besicovitch space with respect to the nonnegative, $\sigma$-finite, c.a. Borel measure $\mu$, and $N_{\lambda}$ stand for standard neighborhoods of $\lambda, c f .2 .5$;

(ii) the measure $M$ be related to $\mu$ as in (9.10);

(iii) $\Lambda_{0}=\{\lambda: \lambda \in \sigma(\mu) \&$

$$
\left.\lim _{N_{\lambda} \rightarrow \lambda}\left|\frac{1}{\mu\left(N_{\lambda}\right)} \int_{N_{\lambda}} M_{\mu}^{\prime}\left(\lambda^{\prime}\right) \mu\left(d \lambda^{\prime}\right)-M_{\mu}^{\prime}(\lambda)\right|_{B}=0\right\},
$$

$\sigma(\mu)$ being the spectrum of $\mu$, 
(iv) $\forall \phi \in \mathfrak{L}_{2}$,

$\Lambda_{\phi} \overline{\bar{d}}\{\lambda: \lambda \in \sigma(\mu) \&$

$$
\left.\lim _{N_{\lambda \rightarrow \lambda}}\left|\frac{1}{\mu\left(N_{\lambda}\right)} \int_{N_{\lambda}} M_{\mu}^{\prime}\left(\lambda^{\prime}\right) \phi\left(\lambda^{\prime}\right) \mu\left(d \lambda^{\prime}\right)-M_{\mu}^{\prime}(\lambda) \phi(\lambda)\right|_{W}=0\right\} .
$$

Then (a) $\Lambda \backslash \Lambda_{0} \in \mathbb{B} \& \mu\left(\Lambda \backslash \Lambda_{0}\right)=0$;

(b) $\forall \phi \in \mathscr{L}_{2}, \quad \Lambda \backslash \Lambda_{\phi} \in ß \& \mu\left(\Lambda \backslash \Lambda_{\phi}\right)=0$.

\section{Integration with respect to c.a.q.i. measures}

In this section

(i) TC is any Hilbert space and $W$ a separable one, both over $\mathbf{C}$;

(ii) $B$ is a $\sigma$-ring over an arbitrary set $\Lambda$;

(iii) $M$ is a $W$-to- $W$, nonnegative, hermitian operator-valued measure on a sub $\delta$-ring $\bigotimes_{0}$ of $\Theta_{1}$ c.a. under the strong

(10.1) operator topology; ${ }^{23}$

(iv) $\mathfrak{L}_{2, W}={ }_{d} L_{2}(\Lambda, \leftrightarrow, M ; W)$ is a pre-Hilbert space in which the linear manifold $s\left(\Lambda, \Theta_{0} ; W\right)$ of $\Theta_{0}$-simple functions on $\Lambda$ to $W$ is e.d.;

(v) $\overline{\mathfrak{L}}_{2, W}$ is the completion of the pre-Hilbert space $\mathfrak{L}_{2, W}$;

(vi) $T$ is a $W$-to-JC, c.a.q.i. measure over $\left(\Lambda, \wp_{0}, M\right)$.

In view of its length we have divided this section into 7 parts labeled A, B, C, D, E, F, G.

A. Our first objective is to define for each $\phi$ in $\mathfrak{L}_{2, W}$ the integral $\int_{\Lambda} T(d \lambda) \phi(\lambda)$, in which the operator-valued measure $T(\cdot)$ acts on the $W$-vector valued integrand $\phi(\cdot),{ }^{24}$ so that it will have the following properties:

$$
\int_{\Delta} T(d \lambda) \phi(\lambda) \in \mathscr{H}
$$

(b) $\left(\int_{\Delta} T(d \lambda) \phi(\lambda), \int_{\Delta} T(d \lambda) \psi(\lambda)\right)_{\mathscr{S C}}=(\phi, \psi)_{M}, \quad c f$. (9.24).

We single out these properties because they entail all the others our integral possesses, as the following lemma makes clear:

10.3. Lemma. Any integral $\int_{\Lambda} T(d \lambda) \phi(\lambda)$ defined for all functions $\phi$ in

${ }^{23} \beta_{0}=\beta_{\mu}$ when $M$ satisfies (9.10), and $\beta_{0}=\beta$ when $M$ satisfies (9.16).

24 A more accurate notation would be $\int_{\Lambda} T(d \lambda)\{\phi(\lambda)\}$. 
a linear manifold $\mathcal{S}$ of $\mathcal{L}_{2, W}$, and having the properties (10.2) has the following properties: $\forall \phi, \psi, \phi_{n} \in S$ and $\forall a, b \in C$,

(a)

$$
\left|\int_{\Lambda} T(d \lambda) \phi(\lambda)\right|_{\mathscr{T C}}^{2}=|\phi|_{M}^{2},
$$

(b) ${ }^{25}$ if for $E \in B^{\text {loc }}, \int_{E} T(d \lambda) \phi(\lambda)={ }_{d} \int_{\Delta} T(d \lambda)\left\{\phi(\lambda) \chi_{E}(\lambda)\right\}$, then $\forall A, B \in B^{\text {loo }}$,

$$
\begin{aligned}
\left(\int_{A} T(d \lambda) \phi(\lambda), \int_{B} T(d \lambda) \psi(\lambda)\right)_{\Im C} & \\
= & \left(\phi \chi_{A}, \psi \chi_{B}\right)_{W}=\int_{A \cap_{B}} \psi(\lambda)^{*} M(d \lambda) \phi(\lambda),
\end{aligned}
$$

(c) $\int_{\Lambda} T(d \lambda)\{a \phi(\lambda)+b \psi(\lambda)\}=a \int_{\Lambda} T(d \lambda) \phi(\lambda)+b \int_{\Lambda} T(d \lambda) \psi(\lambda)$,

(d) $\left|\int_{\Delta} T(d \lambda) \phi_{m}(\lambda)-\int_{\Lambda} T(d \lambda) \phi_{n}(\lambda)\right|_{3 c}=\left|\phi_{m}-\phi_{n}\right|_{M}^{2}$,

(e) $\phi_{n} \rightarrow \phi$ in $\mathfrak{L}_{2, W}$, iff $\int_{\Lambda} T(d \lambda) \phi_{n}(\lambda) \rightarrow \int_{\Lambda} T(d \lambda) \phi(\lambda)$ in $\mathfrak{F}$.

We omit the proof, as this closely resembles that of the corresponding result $[30,5.3]$ for c.a.o.s. measures.

We shall now define the integral in two steps, following the pattern for c.a.o.s. measures $[30, \S 5]$ :

10.4. Definition (Step 1). ${ }^{26}$ For $\phi \in \delta\left(\Lambda, B_{0} ; W\right)$, say $\phi=\sum_{1}^{r} w_{k} \chi_{A_{k}}$, where $w_{k} \in W, A_{k} \in \AA_{0}$,

$$
\int_{\Lambda} T(d \lambda) \phi(\lambda)=\sum_{w \in \mathfrak{R}_{\phi}} T\left(\phi^{-1}\{w\}\right)(w)=\sum_{1}^{r} T\left(A_{k}\right)\left(w_{k}\right) .
$$

A simple computation shows that

$$
\forall \phi \in \delta\left(\Lambda, \Theta_{0} ; W\right), \quad \int_{\Lambda} T(d \lambda) \phi(\lambda) \text { has the properties (10.2). }
$$

Consequently it has all the properties $10.3(\mathrm{a})-(\mathrm{e})$. Now let $\phi \in \mathfrak{L}_{2, W}$. By (10.1) (iv) there exists a sequence $\left(\phi_{n}\right)_{1}^{\infty}$ in $\delta\left(\Lambda, \Theta_{0} ; W\right)$ such that $\phi_{n} \rightarrow \phi$ in $\mathfrak{L}_{2, W}$. This sequence is Cauchy in $\mathfrak{L}_{2, W}$ and therefore by 10.3(d), the sequence $\left(\int_{\Lambda} T(d \lambda) \phi_{n}(\lambda)\right)_{n=1}^{\infty}$ is Cauchy in $\mathcal{T C}$ and so has a limit $x$ in $\mathcal{H}$. Furthermore, if $\left(\phi_{n}\right)_{1}^{\infty}$ is another sequence in $\delta\left(\Lambda, B_{0} ; W\right)$ converging to $\phi$ in $\mathfrak{L}_{2, W}$ then $\phi_{n}-\psi_{n} \rightarrow 0$ in $\mathfrak{L}_{2, W}$, and therefore by $10.3(\mathrm{~d})$

$$
\left|\int_{\Lambda} T(d \lambda) \phi_{n}(\lambda)-\int_{\Lambda} T(d \lambda) \psi_{n}(\lambda)\right|_{\Re C} \rightarrow 0, \quad \text { as } n \rightarrow \infty .
$$

${ }^{25}$ Recall that $B^{100}$ is the $\sigma$-algebra of sets "locally in $B^{1}$ ", i.e. $B^{100}={ }_{d}\{E: E \subseteq \Lambda \&$ $\forall B \in ß, E \cap B \in ß\} \supseteq ß$.

${ }_{26}^{26}$ Recall that $\mathcal{S}\left(\Lambda, \bigotimes_{0} ; W\right)$ denotes the set of $\bigotimes_{0}$-simple functions on $\boldsymbol{\Lambda}$ to $W$, cf. 9.25(a). 
This shows that the limit $x$ depends only on $\phi$, and not on the approximating sequences. The following natural definition is thus unequivocal:

10.6. Definition (Step 2). For a $\phi$ in $\mathfrak{L}_{2, W}$, which is not $\beta_{0}$-simple,

$$
\int_{\Lambda} T(d \lambda) \phi(\lambda)=\lim _{n \rightarrow \infty} \int_{\Lambda} T(d \lambda) \phi_{n}(\lambda),
$$

where $\left(\phi_{n}\right)_{1}^{\infty}$ is any sequence in $\varsigma\left(\Lambda, \beta_{0} ; W\right)$ converging to $\phi$ in $\mathfrak{L}_{2, W}$.

From (10.5) and 10.6 it follows easily that our integral has the properties (10.2), and so by 10.3 , the properties $10.3(\mathrm{a})-(\mathrm{e})$ as well. Introducing the spatial integral

$$
\int_{\Lambda} T(d \lambda)(W)=\left\{\int_{\Lambda} T(d \lambda) \phi(\lambda): \phi \in \mathfrak{L}_{2, W}\right\},
$$

we may sum up its properties in the following theorem:

10.8. ISOMORPHISM THEOREM. The correspondence $\Sigma_{T}: \phi \rightarrow \int_{\Lambda} T(d \lambda) \phi(\lambda)$ is a linear isometry on the pre-Hilbert space $\mathfrak{L}_{2, W}$ onto the linear manifold $\int_{\Lambda} T(d \lambda)(W) \subseteq \mathcal{F}$.

10.9 Corollary. (a) cls. $\int_{\Lambda} T(d \lambda)(W)=\S_{T}$, the subspace of the measure $T(\cdot)$. For adequate $M, c f .9 .9 \& 9.25(\mathrm{a}), \int_{\Lambda} T(d \lambda)(W)=\delta_{T}$.

(b) If the measure $T(\cdot)$ is $\mathcal{H C}$-basic, then cls. $\int_{\Lambda} T(d \lambda)(W)=\mathcal{H}$. If , furthermore, $M$ is adequate, then $\int_{\Lambda} T(d \lambda)(W)=\mathcal{H}$, i.e. roughly speaking, HC is a "continuous sum" of orthogonal "differential subspaces" obtained from the wandering space $W$, cf. 8.6(a).

Proof. (a) Let $x \in \int_{\Lambda} T(d \lambda)(W)$. Then by Defs. $10.4,10.6, x$ is a linear combination $\sum_{1}^{r} T\left(A_{k}\right)\left(w_{k}\right), A_{k} \in \beta_{0}, w_{k} \in W$, or a limit thereof; hence $x \in S_{T}$. Thus

$$
\int_{\mathbf{\Lambda}} T(d \lambda)(W) \subseteq \delta_{T}, \quad \& \text { so cls. } \int_{\mathbf{\Lambda}} T(d \lambda)(W) \subseteq \delta_{T} .
$$

To prove the reverse inclusion, note that by $10.4 \&(10.7)$

$\forall B \in \beta_{0} \& \forall w \in W$,

$$
T(B)(w)=\int_{\Lambda} T(d \lambda)\left\{w \chi_{B}(\lambda)\right\} \in \int_{\Lambda} T(d \lambda)(W),
$$

${ }^{27}$ The notation $\int_{\Lambda} T(d \lambda)(W)$ for the set of all convergent vector integrals $\int_{\Lambda} T(d \lambda)\{\phi(\lambda)\}$, where $\phi(\lambda) \in W$, is a reasonable extension of the standard notation $\sum_{k=0}^{\infty} T^{k}(W)$ for the set of all convergent vector sums $\sum_{k=0}^{\infty} T^{k}\{\phi(k)\}$, where $\phi(k) \in W$ and $T$ is a single operator. The term "spatial integral" seems to be more appropriate than the term "direct integral" used previously in $[28,86]$. 
and so

$$
\forall B \in B_{0}, \quad T(B)(W) \subseteq \int_{\Lambda} T(d \lambda)(W) .
$$

Since the last set is a linear manifold, it follows that

$$
\delta_{T} \underset{d}{=} \subseteq\left\{T(B)(W): B \in B_{0}\right\} \subseteq \text { cl. } \int_{\Lambda} T(d \lambda)(W) .
$$

(1) and (2) yield the first equality in (a). The second follows when $M$ is adequate, for then the domain $\mathfrak{L}_{2, W}$ of the isometry $\Sigma_{T}$ of 10.8 is a Hilbert space, and therefore its range, $\int_{\Lambda} T(d \lambda)(W)$, is closed in $\mathcal{H}$.

This establishes (a). (b) follows trivially.

Thm.10.8 and Cor.10.9(a) subsume the Isomorphism Thm.2.3 for c.a.o.s. measures. Indeed, the latter emerges on taking $W=\boldsymbol{C}$ and $M=\mu$. But even for the most general $W$ the precise connection between the concepts of integration with respect to c.a.q.i. and c.a.o.s. measures is easy to state:

10.10 Triviality. Let (i) $\forall w \in W, \quad \xi_{w}(\cdot)={ }_{d} T(\cdot)(w), \mu_{w w}(\cdot)={ }_{d}$ $(M(\cdot) w, w)_{W} c f .8 .5$; (ii) $\phi \in L_{2}\left(\Lambda, \Theta, \mu_{w w} ; C\right)$. Then

$$
\int_{\Lambda} T(d \lambda)\{\phi(\lambda) w\}=\int_{\Lambda} \phi(\lambda) \xi_{w}(d \lambda) .
$$

Proof. Write $\nu$ for $\mu_{w w}$ and first let $\phi$ be $\oiint_{\nu}$-simple, say $\phi=\sum_{1}^{r} c_{k} \chi_{A_{k}}$, $c_{k} \in \mathrm{C} \& A_{k} \in \Re_{\nu}$. Then

$$
\begin{aligned}
\int_{\Lambda} T(d \lambda)\{\phi(\lambda) w\} & =\sum_{\mathbf{1}}^{r} T\left(A_{k}\right)\left(w c_{k}\right)=\sum_{\mathbf{1}}^{r} c_{k} \xi_{w}\left(A_{k}\right) \\
& =\int_{\mathbf{\Lambda}} \phi(\lambda) \xi_{w}(d \lambda) .
\end{aligned}
$$

Next let

$$
\phi=\lim _{n \rightarrow \infty} \phi_{n} \text { in } L_{2}(\Lambda, \Theta, \nu ; C) \& \phi_{n} \text { be } \Theta_{\nu} \text {-simple. }
$$

Then by $9.25(\mathrm{k})$

$$
\left|w \phi-w \phi_{n}\right|_{M}^{2}=\int_{\Lambda}\left|\phi(\lambda)-\phi_{n}(\lambda)\right|^{2} \nu(d \lambda) .
$$

It follows from (2), (3) that $w \phi=\lim _{n \rightarrow \infty} w \phi_{n}$ in $\mathfrak{L}_{2, W}$, and hence by Thm. 10.8, (1) and (2) 


$$
\begin{aligned}
\int_{\Delta} T(d \lambda)\{w \phi(\lambda)\} & =\lim _{n \rightarrow \infty} \int_{\Lambda} T(d \lambda)\left\{w \phi_{n}(\lambda)\right\} \\
& =\lim _{n \rightarrow \infty} \int_{\Delta} \phi_{n}(\lambda) \xi_{w}(d \lambda)=\int_{\Lambda} \phi(\lambda) \xi_{w}(d \lambda) .
\end{aligned}
$$

10.11. Corollary. The correspondence between the W-to-JC c.a.q.i. measures over the same $\left(\Lambda, \mathrm{B}_{0}, M\right)$ and the induced isometries on $\mathfrak{L}_{2, W}$ to He given by 10.8 is one-one; i.e.

$$
\Sigma_{T_{1}}=\Sigma_{T_{2}} \Rightarrow T_{1}(\cdot)=T_{2}(\cdot) \text { on } \beta_{0} \text {. }
$$

Proof. By Thm.10.8 and Def.10.4, $\forall A \in \wp_{0} \& \forall w \in W$,

$$
\Sigma_{T_{i}}\left(w \cdot \chi_{A}(\cdot)\right)=\int_{\Lambda} T_{i}(d \lambda)\left\{w \chi_{A}(\lambda)\right\}=T_{i}(A)(w), \quad i=1,2
$$

From this the desired implication clearly follows.

B. Our next objective is to show that every isometry $\Sigma$ on $\mathscr{L}_{2, W}$ into $\mathcal{H C}$ hails from a $W$-to- $\mathcal{H C}$ c.a.q.i. measure in the manner of $\mathrm{Thm}$. 10.8. This hinges on the following two lemmas which are clear generalizations of our $[30,5.14]$ and 2.8 :

10.12. LEMMA (EFFECT OF AN ISOMETRY). Let

(i) $V$ be an isometry on $\mathfrak{H}$ into a Hilbert space $\mathfrak{F C}^{\prime}$,

(ii) $\forall A \in \mathrm{B}_{0}, \quad R(A)=\operatorname{Vo}\{T(A)\}$.

Then

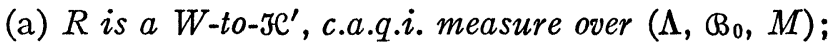

(b) $\quad \forall \phi \in \mathfrak{L}_{2, W}, V\left\{\int_{\Delta} T(d \lambda) \phi(\lambda)\right\}=\int_{\Lambda} R(d \lambda) \phi(\lambda)$.

Proof. (a) Let $A \in \Theta_{0}$. Since $T(A)$ and $V$ are continuous linear operators on $W$ to $\mathfrak{H}$ and on $\mathfrak{H C}$ to $\mathfrak{H C}^{\prime}$, therefore

$R(A)$ is a continuous linear operator on $W$ to $\mathfrak{H C}^{\prime}$.

Also, since $V$ is an isometry, therefore $\forall A, B \in \Theta_{0}$ and $\forall w, w \in W$, $\left(R(A) w, R(B) w^{\prime}\right)_{\mathcal{C}^{\prime}}=\left(T(A) w, T(B) w^{\prime}\right)_{\mathfrak{r}}=\left(M(A \cap B) w, w^{\prime}\right)_{W}$. Hence (a).

(b) is easily verified for $\beta_{0}$-simple $\phi$ on $\Lambda$ to $W$, and thence, since $V$ is an isometry, by the usual limiting argument for any $\phi \in \mathfrak{L}_{2, W}$.

10.13. LEMMA (INDICATOR C.A.Q.I. BASIC MEASURE FOR $\mathfrak{L}_{2, w}$ ). Let 
$\forall A \in B_{0} \& \forall w \in W, \quad\left\{M_{\chi}(A)\right\}(w)={ }_{d} w \chi_{A}(\cdot) .{ }^{28}$

Then, $c f .(9.1)(\mathrm{v})$,

(a) $M_{\chi}(\cdot)$ is a W-to- $\overline{\mathscr{L}}_{2, W}$ basic, c.a.q.i. measure over $\left(\Lambda, \bigotimes_{0}, M\right)$;

(b) $\forall \phi \in \mathfrak{L}_{2, W}, \quad \int_{\Lambda} M_{\chi}(d \lambda) \phi(\lambda)=\phi(\cdot)$.

Proof. (a) Let $A, B \in B_{0}$ and $w, w^{\prime} \in W$. Then to prove (a) we have only to show

$$
\begin{gathered}
w \chi_{A}(\cdot) \in \mathfrak{L}_{2, W} \\
\left(M_{\chi}(A)(w), M_{\chi}(B)(w \cdot)\right) \mathfrak{L}_{2, W}=\left(M(A \cap B)(w), w^{\prime}\right)_{W},
\end{gathered}
$$

and

$$
\forall \bar{\phi} \in \overline{\mathfrak{L}}_{2, W}, \quad \bar{\phi} \in \boldsymbol{s}_{M_{x}} .
$$

Now since $A \in B_{0}$, which is the domain of $M$,

$$
\mu_{w w}(A)=(M(A)(w), w)_{W}=|\{\sqrt{ } M(A)\}(w)|_{W}^{2}<\infty
$$

Hence $\chi_{A} \in L_{2}\left(\Lambda, B, \mu_{w w} ; C\right)$, and (1) follows by $9.25(\mathrm{~h})$.

Next by $9.25(\mathrm{k})$

$$
\begin{aligned}
\operatorname{LHS}(2) & =\left(w \chi_{A}(\cdot), w \chi_{B}^{\prime}(\cdot)\right)_{M}=\int_{\Delta} \chi_{A}(\lambda) \overline{\chi_{B}(\lambda) \mu_{w} w^{\prime}}(d \lambda) \\
& =\mu_{w w^{\prime}}(A \cap B)=\left(M(A \cap B) w, w^{\prime}\right)_{w} .
\end{aligned}
$$

Finally, to prove (3), note that it holds for $\phi \in \delta\left(\Lambda, \wp_{0} ; W\right)$, since

$$
\phi=\sum_{1}^{r} w_{k} \chi_{A_{k}}=\sum_{1}^{r} M_{\chi}\left(A_{k}\right)\left(w_{k}\right) \in \varsigma_{M_{\mathbf{x}}}
$$

But by (10.1)(iv) such functions $\phi$ are e.d. in $\mathfrak{L}_{2, W}$, and therefore they (or rather the corresponding elements $[\phi]$ ) are e.d. in $\overline{\mathfrak{L}}_{2, W}$. Hence (3) holds for any $\Phi$ in $\mathfrak{L}_{2, W}$.

(b) Let $\phi \in S\left(\Lambda, \beta_{0} ; W\right)$, say $\phi=\sum_{1}^{r} w_{k} \chi_{A_{k}}$. Then by Def.10.4 and (4)

$$
\int_{\Lambda} M_{\chi}(d \lambda) \phi(\lambda)=\sum_{1}^{r} M_{\chi}\left(A_{k}\right)\left(w_{k}\right)=\phi
$$

${ }^{28}$ This definition of $M_{x}(\cdot)$ is perfect when $\mathscr{L}_{2, w}$ is a Hilbert space, i.e. when the measure $M$ is adequate. In the general case we should, strictly speaking, write $\left[w x_{A}(\cdot)\right]$ on the RHS of the defining equality, with the stipulation that $[\phi(\cdot)]$ denotes the element in $\overline{\mathfrak{L}}_{2, W}$ corresponding to $\phi(\cdot)$ in $\mathfrak{L}_{2, W}$. Here and in similar situations below we shall identify $\phi(\cdot)$ and $[\phi(\cdot)]$. 
Thus the equality in (b) holds on the linear manifold $s\left(\Lambda, B_{0} ; M\right)$. Since by 10.3 (iv) this manifold is e.d. in $\mathfrak{L}_{2, W}$, the equality holds throughout $\mathfrak{L}_{2, W}$.

10.14. Theorem (a Converse of Thm.10.8). Let

(i) $\Sigma$ be an isometry on $\overline{\mathcal{L}}_{2, W}$ into $\mathfrak{K C}$,

(ii) $\bar{\Sigma}$ be the isometric extension of $\Sigma$ on $\bar{\Sigma}_{2, W}$ to $\mathfrak{K C}$,

(iii) $\forall A \in \beta_{0}, R(A)={ }_{d} \bar{\Sigma} \circ\left\{M_{\chi}(A)\right\}$, where $M_{\chi}(\cdot)$ is as in 10.13 . Then

(a) $R$ is a W-to-He, c.a.q.i. measure over $\left(\Lambda, \Theta_{0}, M\right)$;

(b) $\Sigma=\Sigma_{R}$, i.e. $\forall \phi \in \mathcal{L}_{2, W}, \Sigma(\phi)=\int_{\Lambda} R(d \lambda) \phi(\lambda)$.

PRoof. (a) follows from $10.12(\mathrm{a})$, since by $10.13(\mathrm{a}) M_{\chi}(\cdot)$ is a $W$-to- $\bar{\aleph}_{2, W}$, c.a.q.i. measure over $\left(\Lambda, \beta_{0}, M\right)$, and $\bar{\Sigma}$ is an isometry on $\overline{\mathfrak{L}}_{2, W}$ into $\mathfrak{H}$.

(b) Let $\phi \in \mathfrak{L}_{2, w}$. Then

$$
\begin{aligned}
\int_{\Lambda} R(d \lambda) \phi(\lambda) & =\bar{\Sigma}\left\{\int_{\Lambda} M_{\chi}(d \lambda) \phi(\lambda)\right\}, & & \text { by (ii) } \& 10.12(\mathrm{~b}), \\
& =\bar{\Sigma}(\phi), & & \text { by } 10.13(\mathrm{~b}), \\
& =\Sigma(\phi), & & \text { since } \bar{\Sigma}=\Sigma \text { on } \mathfrak{L}_{2, W} .
\end{aligned}
$$

C. To simplify our enunciations we shall now assume that the measure $M$ of (10.1)(iii) is adequate according to Criterion 9.9. Then by the Isomorphism Thm.10.8 and Cor.10.9(a) to each $x \in \mathcal{F}$ there corresponds a unique function $\phi_{x} \in \mathfrak{L}_{2, W}$ such that $P_{S_{T}}(x)=\Sigma_{T}\left(\phi_{x}\right)$ and so

$$
\phi_{x}=\Sigma_{T}^{-1} P s_{T}(x)=\Sigma_{T}^{*}(x) .
$$

This brings up the question of finding $\phi_{x}(\cdot)$ for a given $x \in \mathcal{F}$. The corresponding question for c.a.o.s. measures is answered by our Projection Theorems $[30,5.10]$ and 2.6. The analogues of these theorems for c.a.q.i. measures emerge from the following result:

10.16. Theorem. Let the measure $M$ of (9.1)(iii) be adequate. Then

(a) $\forall B \in \leftrightarrow_{0} \& \forall \phi \in \mathscr{L}_{2, W}, T(B)^{*}\left\{\int_{\Delta} T(d \lambda) \phi(\lambda)\right\}=\int_{B} M(d \lambda) \phi(\lambda)$.

(b) $\forall B \in \beta_{0} \& \forall x \in \mathfrak{H C}, \quad \zeta_{x}(B)=\bar{d} T(B)^{*}(x)=\int_{B} M(d \lambda)\left\{\Sigma_{T}^{*}(x)\right\}(\lambda)$.

Proof. (a) Case (i). Let $\phi$ be $\circledast_{0}$-simple, say 


$$
\phi=\sum_{1}^{r} w_{k} \chi_{A_{k}}, \quad w_{k} \in W \& A_{k} \in \Theta_{0} .
$$

Then by Defs.10.4 \& 8.2(iii), $\forall B \in B_{0}$

$$
T(B)^{*}\left\{\int_{\Lambda} T(d \lambda) \phi(\lambda)\right\}=\sum_{1}^{r} M\left(B \cap A_{k}\right)\left(w_{k}\right) .
$$

Also, by $9.25(\mathrm{c})$,

$$
\int_{B} M(d \lambda)\{\phi(\lambda)\}=\sum_{1}^{r} M\left(B \cap A_{k}\right)\left(w_{k}\right) .
$$

Case (ii). Let $\phi=\lim _{n \rightarrow \infty} \phi_{n}$ in $\mathfrak{L}_{2, W}$, where $\phi_{n}$ are $B_{0}$-simple. Then using in succession Def. 10.6, the continuity of the operator $T(B)$ *, result (a) for Case (i), and $9.25(\mathrm{~g})$, we get

$$
\begin{aligned}
T(B)^{*}\left\{\int_{\Lambda} T(d \lambda) \phi(\lambda)\right\} & =T(B)^{*}\left\{\lim _{n \rightarrow \infty} \int_{\Lambda} T(d \lambda) \phi_{n}(\lambda)\right\} \\
& =\lim _{n \rightarrow \infty} T(B)^{*}\left\{\int_{\Lambda} T(d \lambda) \phi_{n}(\lambda)\right\} \\
& =\lim _{n \rightarrow \infty} \int_{B} M(d \lambda) \phi_{n}(\lambda) \\
& =\int_{B} M d(\lambda) \phi(\lambda) .
\end{aligned}
$$

Thus (a).

(b) Let $x \in \mathcal{H}$ and $B \in \Re_{0}$. Then

$$
\begin{aligned}
\zeta_{x}(B) & =T(B)^{*}\left\{P s_{T}(x)\right\}, & & \text { by }(8.10) \\
& =T(B)^{*}\left\{\Sigma_{T}\left(\phi_{x}\right)\right\} & & \text { by def. of } \phi_{x} \\
& =\int_{B} M(d \lambda) \phi_{x}(\lambda) & & \text { by (a) } \\
& =\int_{B} M(d \lambda)\left\{\Sigma_{T}^{*}(x)\right\}(\lambda) & & \text { by }(10.15) .
\end{aligned}
$$

The penultimate equation in the last proof shows that in a sense $\phi_{x}$ is the Radon-Nikodým derivative of the $W$-valued, c.a. measure $\zeta_{x}$ with respect to the $W$-to- $W$ operator-valued measure $M(\cdot)$, and we could write:

$$
\Sigma_{T}^{*}(x)=\phi_{x}=\left[(d M)^{-1} d \zeta_{x}\right]
$$


This statement corresponds to the Projection Theorem $[30,5.10]$ of c.a.o.s. measure theory. It is convenient, however, to reserve this title for the following more useful version obtainable from (10.16') when the measure $M$ is the indefinite integral of a nonnegative measure:

10.17. Projection Theorem (for $M$ as in (9.10) \& (9.15)). Let

(i) the measures $M$ \& $\mu$ satisfy (9.10) \& (9.15),

(ii) $\forall x \in \mathcal{F}, \phi_{x}(\cdot)$ be the unique function in $\mathfrak{L}_{2, W}$ such that

$$
P s_{T}(x)=\int_{\Lambda} T(d \lambda) \phi_{x}(\lambda),
$$

(iii) $x \in \mathfrak{H C}, \& \quad \forall B \in \mathbb{B}_{\mu}, \zeta_{x}(B)={ }_{d} T(B) *(x)$.

\section{Then}

(a) $\quad M_{\mu}^{\prime}(\cdot)\left\{\phi_{x}(\cdot)\right\}=d \zeta_{x} / d \mu(\cdot)$;

(b) when $M(\cdot)=\mu(\cdot) I, \quad \phi_{x}(\cdot)=d \zeta_{x} / d \mu(\cdot)$.

Proof. (a) By Thm.10.16(b) and Lma.9.28(c), $\forall B \in B_{\mu}$,

$$
\zeta_{x}(B)=\int_{B} M(d \lambda) \phi(\lambda)=\int_{B} M_{\mu}^{\prime}(\lambda)\{\phi(\lambda)\}_{\mu}(d \lambda),
$$

from which (a) is immediate.

(b) follows, since $M_{\mu}^{\prime}(\lambda)=I$, when $M(\cdot)=\mu(\cdot) I$.

If, further, $(\Lambda, \beta)$ is a Besicovitch space with respect to $\mu$, the formulae in 10.17 provide recipes for finding $\phi_{x}$ :

10.18. Projection Theorem (for Besicovitch $\Lambda \& M$ AS IN (9.10) $\&(9.15))$. Let

(i) $(\Lambda, B)$ be a Besicovitch space with respect to the nonnegative, $\sigma$-finite, c.a., Borel measure $\mu$, and $N_{\lambda}$ be a standard neighborhood of $\lambda$, cf. 2.5,

(ii) $x \in \mathfrak{H C}$, and $M \& \phi_{x}$ be as in 10.17 (i)\&(ii).

Then

(a) $\quad M_{\mu}^{\prime}(\lambda)\left\{\phi_{x}(\lambda)\right\}=\lim _{N_{\lambda} \rightarrow \lambda}\left\{\frac{1}{\mu\left(N_{\lambda}\right)} T\left(N_{\lambda}\right)^{*}(x)\right\}, \quad$ a.e. $(\mu)$ on $\Lambda$;

(b) in case $M(\cdot)=\mu(\cdot) I$,

$$
\phi_{x}(\lambda)=\lim _{N_{\lambda} \rightarrow \lambda}\left\{\frac{1}{\mu\left(N_{\lambda}\right)} T\left(N_{\lambda}\right)^{*}(x)\right\}, \text { a.e. }(\mu) \text { on } \Lambda .
$$

Proof. (a) Let $\zeta_{x}$ be as in 10.17(iii). Then as in the last proof 


$$
\forall B \in \mathbb{B}_{\mu}, \quad \zeta_{x}(B)=\int_{B} M_{\mu}^{\prime}(\lambda)\left\{\phi_{x}(\lambda)\right\} \mu(d \lambda) .
$$

Since $M_{\mu}^{\prime}(\cdot)\left\{\phi_{x}(\cdot)\right\} \in L_{1}^{\text {loc }}(\Lambda, B, \mu ; W)$, (a) is immediate from the Differentiation Thm.9.30(b).

(b) follows, since $M_{\mu}^{\prime}(\lambda)=I$ when $M(\cdot)=\mu(\cdot) I$.

The following result is of some interest.

10.19. Corollary. Let

(i) be as in the last theorem,

$$
\begin{aligned}
M(\cdot) & =\mu(\cdot) I, \\
\lambda \in \sigma(\mu) \& L_{\lambda} & =\operatorname{sim}_{N_{\lambda \rightarrow \lambda}} \frac{T\left(N_{\lambda}\right)^{*}}{\mu\left(N_{\lambda}\right)} .
\end{aligned}
$$

Then $L_{\lambda}$ is a continuous linear operator on JC to $W$, iff $\{\lambda\}$ is an atom of $\mu$, i.e. $\mu\{\lambda\}>0$.

Note. Thus $L_{\lambda}$ will in general be a discontinuous linear operator, and dom. $\left(L_{\lambda}\right) \subset \mathcal{H C}$.

Proof. For a (fixed) $\lambda \in \sigma(\mu)$, let $L_{\lambda}$ be continuous and $\operatorname{dom} .\left(L_{\lambda}\right)=$ He. Then

$$
L_{\lambda}^{*} \text { is a continuous linear operator on } W \text { to } \mathfrak{F e} \text {. }
$$

Hence $\forall w \in W \& \forall x \in \mathfrak{x}$,

$$
\begin{aligned}
\left(x, L_{\lambda}^{*}(w)\right)_{\mathscr{S C}} & =\left(L_{\lambda}(x), w\right)_{W}=\lim _{N_{\lambda} \rightarrow \lambda}\left(\frac{T\left(N_{\lambda}\right)^{*}}{\mu\left(N_{\lambda}\right)}(x), w\right)_{W} \\
& =\lim _{N_{\lambda} \rightarrow \lambda}\left(x, \frac{T\left(N_{\lambda}\right)}{\mu\left(N_{\lambda}\right)}(w)\right)_{\mathscr{S C}}=\lim _{N_{\lambda} \rightarrow \lambda}\left(x, \frac{\xi_{w}\left(N_{\lambda}\right)}{\mu\left(N_{\lambda}\right)}\right)_{\Im C},
\end{aligned}
$$

where, cf. 8.5(a),

$\xi_{w}(\cdot)={ }_{d} T(\cdot)$ w is a KC-val., c.a.o.s. meas. over $\left(\Lambda, \AA,|w|{ }^{2} \mu(\cdot)\right)$.

By (1) $\quad \operatorname{wim}_{N_{\lambda \rightarrow \lambda}} \xi_{w}\left(N_{\lambda}\right)$ exists \& $=L_{\lambda}^{*}(w)$.

Hence by $[30,6.6] \mu\{\lambda\}>0$.

Next let $\mu\{\lambda\}>0$. Since $\mu\left(N_{\lambda}\right) \rightarrow \mu\{\lambda\}$ and so by $8.5(\mathrm{e}) T\left(N_{\lambda}\right)$ $\rightarrow T\{\lambda\}$ under the Banach norm $|\cdot|_{B}$, it follows that as $N_{\lambda} \rightarrow \lambda$,

$$
\frac{T\left(N_{\lambda}\right)}{\mu\left(N_{\lambda}\right)} \rightarrow \frac{T\{\lambda\}}{\mu\{\lambda\}} \& \frac{T\left(N_{\lambda}\right)^{*}}{\mu\left(N_{\lambda}\right)} \rightarrow \frac{T\{\lambda\}^{*}}{\mu\{\lambda\}}
$$


under the norm $|\cdot|_{B}$. Thus

$$
L_{\lambda}=\frac{T\{\lambda\}^{*}}{\mu\{\lambda\}}=\text { a continuous lin. oper. on to to } W \text {. }
$$

D. At this stage it is convenient to revert to the case in which the measure $M$ is subject to 9.1 (iii) alone, and to comment on the notion of indefinite integral of a function $\phi$ in $\mathfrak{L}_{2, w}$ with respect to our c.a.q.i. measure $T(\cdot)$ :

10.20. Definition.

(a) $\forall \phi \in \mathfrak{L}_{2, W}$, the $\mathfrak{H}$-valued set-function $\theta_{\phi}(\cdot)$ such that $(c f .10 .3(\mathrm{~b})$ )

$$
\forall E \in B^{\mathrm{loc}}, \quad \theta_{\phi}(E)=\int_{E} T(d \lambda) \phi(\lambda)
$$

is called the indefinite integral of $\phi$ with respect to $T$.

(b) $\forall \phi, \psi \in \mathfrak{L}_{2, W}$, the C-valued set-function $\nu_{\phi, \psi}$ such that

$$
\forall E \in \beta^{\text {loc }}, \quad \nu_{\phi, \psi}(E)=\left(\theta_{\phi}(E), \theta_{\psi}(E)\right)_{3 C}
$$

is called the covariance measure of $\boldsymbol{\theta}_{\phi}, \boldsymbol{\theta}_{\mathbf{\psi}}$.

10.21. Triviality. Let $\phi, \psi \in \mathfrak{L}_{2, W}$. Then

(a) $\nu_{\phi, \psi}(\cdot)$ is a bounded, complex-valued, c.a. measure on the $\sigma$-algebra $B^{\text {loc }}$; and $\nu_{\phi, \phi}$ is nonnegative;

(b) $\theta_{\phi}(\cdot)$ is a bounded, HC-valued, c.a.o.s. measure over $\left(\Lambda, B^{\text {loc }}, \nu_{\phi, \phi}\right)$;

(c) $\theta_{\phi}(\cdot), \theta_{\psi}(\cdot)$ are biorthogonal; more fully:

$$
\forall A, B \in B^{\text {loc }}, \quad\left(\theta_{\phi}(A), \theta_{\psi}(B)\right)_{\mathcal{H C}}=\nu_{\phi, \psi}(A \cap B) .
$$

Proof. (a) Let $A, B, E \in B^{\text {loc. Then by } 10.3(\mathrm{~b})}$

$$
\left(\theta_{\phi}(A), \theta_{\psi}(B)\right)_{\mathcal{F C}}=\int_{A \cap_{B}} \psi(\lambda) * M(d \lambda) \phi(\lambda) .
$$

On setting $A=B=E$, this becomes

$$
\nu_{\phi, \psi}(E)=\int_{E} \psi(\lambda)^{*} M(d \lambda) \phi(\lambda),
$$

from which (a) is immediate, cf. 9.25(f).

(b) \& (c). Combining (1) and (2), we get

$$
\left(\theta_{\phi}(A), \theta_{\psi}(B)\right)_{\mathcal{H C}}=\nu_{\phi, \psi}(A \cap B),
$$

which yields (c); and on setting $\psi=\phi$, (b) follows. 
From the fact that the measure $\theta_{\phi}$ is c.a., it follows that

$$
\begin{aligned}
\forall n & \geqq 1, E_{n} \in \mathbb{B}^{\mathrm{loc},} E_{n} \subseteq E_{n+1} \& \bigcup_{1}^{\infty} E_{n}=E \\
& \Rightarrow \forall \phi \in \mathfrak{L}_{2, W}, \quad \lim _{n \rightarrow \infty} \int_{E_{n}} T(d \lambda) \phi(\lambda)=\int_{E} T(d \lambda) \phi(\lambda) .
\end{aligned}
$$

From the fact that the measures $\theta_{\phi}, \theta_{\psi}$ are biorthogonal, it follows that

$$
\begin{aligned}
A, B \in \mathbb{Q}^{\text {loo }} \& A \| B & \Rightarrow \\
\forall \phi, \psi & \in \mathfrak{L}_{2, W}, \quad \int_{A} T(d \lambda) \phi(\lambda) \perp \int_{B} T(d \lambda) \psi(\lambda) .
\end{aligned}
$$

Extending the notion of spatial integral given in (10.7) by letting

$$
\forall E \in B^{\text {loc }}, \quad \int_{E} T(d \lambda)(W)=\left\{\int_{E} T(d \lambda) \phi(\lambda): \phi \in \mathscr{L}_{2, W}\right\},
$$

it follows easily from 10.3 that the $\int_{E} T(d \lambda)(W)$ are linear manifolds in $\mathcal{H}$. Concerning these we have the following result:

10.25. Theorem (DECOMPosition of SPATIAL INTEgRal). (a) Let $\forall n \geqq 1, E_{n} \in \Theta^{1 \mathrm{loc}}, E_{n}$ be $\|$, and $\cup_{1}^{\infty} E_{n}=E$. Then

$$
\int_{E} T(d \lambda)(W)=\sum_{n=1}^{\infty} \int_{E_{n}} T(d \lambda)(W), \quad \int_{E_{m}} T(d \lambda)(W) \perp \int_{E_{n}} T(d \lambda)(W) ;
$$

i.e. letting $\mathfrak{T}(E)=$ cls. $\int_{E} T(d \lambda)(W), \mathfrak{T}(\cdot)$ is a c.a.o.s. subspace-valued measure on $\mathrm{B}^{\mathrm{loc}}$ for the Hilbert space $\mathrm{S}_{T}$.

(b) Let $\forall E \in B^{\text {loc }}, Q(E)$ be the projection on $\varsigma_{T}$ onto $\operatorname{Tr}(E)$. Then $Q(\cdot)$ is a spectral measure on $\mathbb{B}^{\text {loc }}$ for the Hilbert space $S_{T}$, and

$$
\forall \phi \in \mathfrak{L}_{2, W}, \quad Q(E)\left\{\int_{\Lambda} T(d \lambda) \phi(\lambda)\right\}=\int_{E} T(d \lambda) \phi(\lambda) .
$$

(c) The isometry $\Sigma_{T}: \phi \rightarrow \int_{\Lambda} T(d \lambda) \phi(\lambda)$ on $\mathfrak{L}_{2, W}$ onto $\int_{\Lambda} T(d \lambda)(W)$ carries the operation $M_{\chi E}$ of multiplication by $\chi_{E}$ into the projection $Q(E)$, i.e.

$$
\forall E \in \mathbb{B}^{\text {loc }}, \quad \text { Rstr. } \int_{\Delta^{T(d \lambda)(W)}} Q(E)=\Sigma_{T} \circ M_{\chi_{E}} \circ \Sigma_{T}^{-1}
$$

Proof. (a) The orthogonality relations in (a) are clear from (10.23) and (10.24). The equality in (a) follows from these relations, the 
c.a.o.s. nature of the measures $\theta_{\phi}$, cf. 10.21(b), and the equality $\mathfrak{L}_{2, W}(E)=\sum_{1}^{\infty} \mathfrak{L}_{2, W}\left(E_{n}\right)$ noted in $9.25(\mathrm{e})$.

(b) The spectrality of the measure $Q(\cdot)$ is clear from (a). Next, let $E \in B^{\text {loo }}$ and $\phi \in \mathfrak{L}_{2, W}$. Since

and

$$
\int_{E} T(d \lambda) \phi(\lambda) \in \mathscr{T}(E)
$$

$$
\int_{\Lambda} T(d \lambda) \phi(\lambda)-\int_{E} T(d \lambda) \phi(\lambda)=\int_{\Lambda \backslash E} T(d \lambda) \phi(\lambda) \perp \mathscr{M}(E),
$$

the equality in (b) is immediate.

(c) $\forall E \in \mathbb{B}^{\text {loo }} \& \forall \phi \in \mathfrak{L}_{2, W}$,

$$
\begin{aligned}
\left\{Q(E) \circ \Sigma_{T}\right\}(\phi) & =Q(E)\left\{\int_{\Lambda} T(d \lambda) \phi(\lambda)\right\}=\int_{E} T(d \lambda) \phi(\lambda) \\
& =\int_{\Lambda} T(d \lambda)\left\{M_{\chi_{E}} \phi\right\}(\lambda)=\left(\Sigma_{T} \circ M_{\chi_{E}}\right)(\phi) .
\end{aligned}
$$

Thus, $Q(E) \circ \Sigma_{T}=\Sigma_{T} \circ M_{\chi_{E}}$ on $\mathfrak{L}_{2, W}$. Since $\Sigma_{T}$ is an isometry on $\mathfrak{L}_{2, W}$ onto $\int_{\Lambda} T(d \lambda)(W)$, the required equality clearly follows.

The concept of indefinite integral can be extended: with any Bmeasurable function $\phi$ on $\Lambda$ to $W$ we can associate a c.a.o.s. measure $\theta_{\phi}$ and we can convert any integral with respect to this c.a.o.s. measure into one with respect to the c.a.q.i. measure $T$. This is shown in the following theorem:

10.26. Theorem. Let

(i) $\phi$ be any B-measurable function on $\Lambda$ to $W$,

(ii) $B_{\phi}$ and $\nu_{\phi \phi}$ be defined as in 9.26(iii), (iv),

(iii) $\forall B \in B_{\phi}, \theta_{\phi}(B)={ }_{d} \int_{\Lambda} T(d \lambda)\left\{\phi(\lambda) \chi_{B}(\lambda)\right\}$.

Then (a) $\theta_{\phi}$ is a JC-valued, c.a.o.s. measure over $\left(\Lambda, \sigma\left(B_{0}\right), \tilde{\nu}\right)$, where $\tilde{\nu}$ is the Hahn extension of $\nu_{\phi \phi}$ to the $\sigma-r i n g ~ \sigma\left(B_{\phi}\right)$ generated by $B_{\phi} ;{ }^{29}$

(b)

$$
\begin{gathered}
\forall f \in L_{2}\left(\Lambda, \sigma\left(\bigotimes_{\phi}\right), \tilde{\nu} ; \boldsymbol{C}\right), \quad f(\cdot) \phi(\cdot) \in \mathfrak{L}_{2, W} \quad \& \\
\int_{\Lambda} f(\lambda) \theta_{\phi}(d \lambda)=\int_{\Lambda} T(d \lambda)\{f(\lambda) \phi(\lambda)\} .
\end{gathered}
$$

Proof. (a) We have to depart a little from the proof of 10.21(a). $\forall A, B \in \Re_{\phi}$, i.e. cf. 9.26(b), $\forall A, B \in \Re_{\tilde{v}}$, we have

${ }^{29} \mathrm{We}$ recall, cf. $9.26(\mathrm{a})$, that $\nu_{\phi \phi}$ is a finite (possible unbounded) nonnegative, c.a. measure on the $\delta$-ring $B_{\phi}$. 


$$
\begin{aligned}
\left(\theta_{\phi}(A), \theta_{\phi}(B)\right)_{\mathcal{H C}} & =\left(\phi \chi_{A}, \phi \chi_{B}\right)_{M}, & & \text { by }(10.12)(\mathrm{b}) \\
& =\int_{\Lambda} \chi_{A} \cap_{B}(\lambda) \phi(\lambda) * M(d \lambda) \phi(\lambda), & & \text { by }(9.24) \\
& =\tilde{\nu}_{\phi \phi}(A \cap B), & & \text { cf. } 9.25 \text { (iii). }
\end{aligned}
$$

(a) follows from this by Def.2.1.

(b) Let $f \in L_{2}\left(\Lambda, \sigma\left(\Theta_{\phi}\right), \tilde{\nu} ; C\right)$. Then as asserted in 9.26(c), $f(\cdot) \phi(\cdot) \in \mathfrak{L}_{2, W}$. Next, the equality in (c) is easily verified for $\beta_{\tilde{i}}-$ simple $f$, i.e. cf. $9.26(\mathrm{~b})$, for $\beta_{\phi}$-simple $f$. For an arbitrary $f \in$ $L_{2}\left(\Lambda, \sigma\left(\Theta_{\phi}\right), \tilde{\nu} ; C\right)$, we consider a sequence of $\Theta_{\phi}$-simple functions $f_{n}$ converging to $f$ in the topology of this Hilbert space. The corresponding sequence $\left(f_{n} \phi_{n}\right)_{1}^{\infty}$ then converges to $f \phi$ in the pre-Hilbert space $\mathfrak{L}_{2, W}$, and the desired equality easily follows.

E. We turn next to the c.a.q.i. measure induced by a transformation of the measure-space $\Lambda$ or of the Hilbert space $W$. The following result is obvious:

10.27. Triviality. Let

(i) $T$ be a W-to-He, c.a.q.i. measure over $\left(\Lambda, \beta_{0}, M\right)$,

(ii) $\theta$ be a function on $\Lambda$ into a space $\bar{\Lambda}$,

(iii) $\quad \tilde{B} \underset{d}{=}\left\{\widetilde{B}: \widetilde{B} \subseteq \tilde{\Lambda} \& \theta^{-1}(\widetilde{B}) \in B\right\}, \quad \tilde{M}=M \circ \theta^{-1}$

$$
\tilde{B}_{0}=\left\{\widetilde{B}: \widetilde{B} \subseteq \tilde{\Lambda} \& \theta^{-1}(\widetilde{B}) \in \Theta_{0}\right\} .
$$

Then To $\theta^{-1}$ is a W-to-HC, c.a.q.i. measure over $\left(\tilde{\Lambda}, \tilde{\Theta}_{0}, \tilde{M}\right)$.

10.28. Theorem (substitution RULE). Let (i)-(iii) be as in 10.27,

$$
\tilde{\mathscr{L}}_{2, W}=L_{2}(\tilde{\Lambda}, \tilde{\Theta}, \tilde{M} ; W) .
$$

Then

$$
\begin{gathered}
\tilde{\phi} \in \tilde{\mathfrak{L}}_{2, W} \quad \text { iff } \tilde{\phi} \circ \theta \in \mathfrak{L}_{2, W} \\
\int_{\tilde{\Lambda}}\left(T \circ \theta^{-1}\right)(d \tilde{\lambda}) \tilde{\phi}(\tilde{\lambda})=\int_{\Lambda} T(d \lambda) \tilde{\phi}\{\theta(\lambda)\},
\end{gathered}
$$

in the sense that if either integral exists, then so does the other, and the two are equal.

Proof. This is proved first for $\tilde{B}_{0}$-simple functions $\tilde{\phi}$, and then by the usual limiting argument for any $\tilde{\phi} \in \mathfrak{L}_{2, W}$. As the proof parallels that of $[30,5.19]$, we omit the details. 
For transformations of the space $W$ we have the following result:

10.29. TheOREM. Let

(i) $S$ be a continuous linear operator on a Hilbert space $W^{\prime}$ to $W$,

(ii) $\forall B \in \Re_{0}, R(B)=T(B) \circ S, N(B)=S^{*} \circ M(B) \circ S$.

Then

(a) $R(\cdot)$ is a $W^{\prime}$-to-He, c.a.q.i. measure over $\left(\Lambda, \Theta_{0}, N\right), c f .9 .27$ (a);

(b) $\quad \forall \psi \in L_{2}\left(\Lambda, \Theta, N ; W^{\prime}\right), \quad S\{\psi(\cdot)\} \in \mathcal{L}_{2, W} \quad \&$

$$
\int_{\Lambda} R(d \lambda) \psi(\lambda)=\int_{\Lambda} T(d \lambda)[S\{\psi(\lambda)\}]
$$

Proof. (a) In view of 9.27 (a), the result (a) is clear from the equality,

$$
\forall A, B \in \Theta_{0}, \quad R(B)^{*} R(A)=S^{*} T(B)^{*} T(A) S=N(A \cap B) .
$$

(b) Let $\psi \in \mathfrak{L}_{2}\left(\Lambda, B, N ; W^{\prime}\right)=\mathfrak{L}_{2, W^{\prime}}$. Then as asserted in $9.27(\mathrm{~b})$, $S\{\psi(\cdot)\} \in \mathscr{L}_{2, W}$. Next, the equality in (b) is easily verified for $\psi \in \mathcal{S}\left(\Lambda, \Theta_{0} ; W^{\prime}\right)$. For an arbitrary $\psi \in \mathcal{L}_{2, W^{\prime}}$, we consider a sequence of functions $\psi_{n}$ in $s\left(\Lambda, Q_{0} ; W^{\prime}\right)$ converging to $\psi$ in the topology of this pre-Hilbert space. The corresponding sequence $\left(S\left\{\psi_{n}(\cdot)\right\}\right)_{1}^{\infty}$ is then in $\delta\left(\Lambda, \Theta_{0} ; W\right)$ and converges to $S\{\psi(\cdot)\}$ in the pre-Hilbert space $\mathfrak{L}_{2, w}$. From this the desired equality follows easily.

Note. By extending our notion of spatial integral (10.7) to cover subspaces $W_{0}$ of $W$, viz.

$$
\int_{\Lambda} T(d \lambda)\left(W_{0}\right)=\left\{\int_{T} T(d \lambda) \phi(\lambda): \phi \in \mathfrak{L}_{2, W_{0}}\right\},
$$

we can assert that in 10.29

$$
\int_{\Lambda} R(d \lambda)\left(W^{\prime}\right)=\int_{\Lambda} T(d \lambda)\{c l s . \text { Range } S\} .
$$

F. Our treatment so far has been basis-free. But when a basis for $W$ is given, it is sometimes useful to formulate our results in terms of the action of the c.a.q.i. measure $T(\cdot)$ on the basic vectors of $W$. For instance, the expression in 10.18(b) for $\phi_{x}(\cdot)$ when $M(\cdot)=\mu(\cdot) I$ and $(\Lambda, B)$ is a Besicovitch space takes the following form:

10.30. Corollary. Let

$\Lambda, \AA, \mu, N_{\lambda}$ be as in Thm.10.18,

(iv) $M(\cdot)=\mu(\cdot) I$, $\left(w_{j}, j \in J\right)$ be an o.n. basis for $W$,

Then $\forall x \in \mathcal{H C} \& \forall \lambda \in \Lambda_{\phi_{x}}, c f .9 .30$ (iv), 


$$
\phi_{x}(\lambda)=\sum_{j \in J} \lim _{N_{\lambda} \rightarrow \lambda}\left(x, \frac{\xi_{j}\left(N_{\lambda}\right)}{\mu\left(N_{\lambda}\right)}\right)_{\mathscr{T}} w_{j} .
$$

Proof. In view of (iii), we need only show that given $j$ in $J$,

$$
\left(\phi_{x}(\lambda), w_{j}\right)_{W}=\lim _{N_{\lambda} \rightarrow \lambda}\left(x, \frac{\xi_{j}\left(N_{\lambda}\right)}{\mu\left(N_{\lambda}\right)}\right)_{\Im C}, \quad \forall \lambda \in \Lambda_{\phi_{x}} .
$$

But by Thm.10.18(b)

$$
\phi_{x}(\lambda)=\lim _{N_{\lambda} \rightarrow \lambda} \frac{1}{\mu\left(N_{\lambda}\right)} T\left(N_{\lambda}\right)^{*}(x) ;
$$

and obviously,

$$
\begin{aligned}
\left(\frac{1}{\mu\left(N_{\lambda}\right)} T\left(N_{\lambda}\right)^{*}(x), w_{j}\right)_{W} & =\left(x, \frac{1}{\mu\left(N_{\lambda}\right)} T\left(N_{\lambda}\right)\left(w_{j}\right)\right)_{\mathcal{F C}} \\
& =\left(x, \frac{\xi_{j}\left(N_{\lambda}\right)}{\mu\left(N_{\lambda}\right)}\right)_{\mathcal{H C}} .
\end{aligned}
$$

(1) clearly follows from (2) and (3).

We shall now show that given an orthonormal basis for $W$, our c.a.q.i. integral $\int_{\Lambda} T(d \lambda) \phi(\lambda)$ can, in a large number of cases, be expressed as a sum of c.a.o.s. integrals derived from the basis:

10.31. Theorem. Let

(i) the measures $M \& \mu$ satisfy (9.10) \& (9.15),

(ii) $\phi \in \mathcal{L}_{2, W}$ be such that $\left|\sqrt{ } M_{\mu}^{\prime}(\cdot)\right|_{B}|\phi(\cdot)|_{W} \in L_{2}(\Lambda, \Theta, \mu ; C)$,

(iii) $\left(w_{j}, j \in J\right)$ be an o.n. basis for $W$,

(iv) $\forall j \in J, \quad \xi_{j}(\cdot) \overline{\bar{d}} T(\cdot)\left(w_{j}\right), \mu_{j}(\cdot) \overline{\bar{d}}\left(M(\cdot)\left(w_{j}\right), w_{j}\right)_{W}$.

Then

(a) $\quad \forall j \in J,\left(\phi(\cdot), w_{j}\right)_{W} \in L_{2}\left(\Lambda, \Theta, \mu_{j} ; C\right)$;

(b) $\quad \phi=\sum_{j \in J}\left(\phi(\cdot), w_{j}\right)_{W} w_{j}$ in $\mathfrak{L}_{2, W} ;^{30}$

(c) $\quad \int_{\Lambda} T(d \lambda) \phi(\lambda)=\sum_{j \in J} \int_{\Lambda}\left(\phi(\lambda), w_{j}\right)_{W} \xi_{j}(d \lambda)$ in $\mathfrak{F}$.

Note. The restriction on $M$ imposed in (ii) will be automatically fulfilled for all $\phi$ in $\mathcal{L}_{2, W}$ by a large class of measures $M$, e.g. for $M(\cdot)=\mu(\cdot) I$.

Proof. (a) By (9.11')

so I.e. $\forall \in>0, \exists$ finite $J_{\epsilon} \subset J \ni J_{\epsilon} \subseteq J_{0} \subset J$ \& $J_{0}$ finite

$$
\Rightarrow\left|\sum_{j \in J_{0}}\left(\phi(\cdot), w_{j}\right) w w_{j}-\phi(\cdot)\right|_{M}<\epsilon .
$$


(1) $\int_{\Lambda}\left|\left(\phi(\lambda), w_{j}\right)_{W}\right|^{2} \mu_{j}(d \lambda)=\int_{\Lambda}\left|\left(\phi(\lambda), w_{j}\right)_{W}\right|^{2}\left(M_{\mu}^{\prime}(\lambda)\left(w_{j}\right), w_{j}\right)_{W} \mu(d \lambda)$.

But $\phi(\lambda)$ being in $W$, it follows from (iii) that

(2) $\phi(\lambda)=\sum_{j \in J}\left(\phi(\lambda), w_{j}\right) w w_{j} \&|\phi(\lambda)|_{w}^{2}=\sum_{j \in J}\left|\left(\phi(\lambda), w_{j}\right)_{W}\right|^{2}$.

Hence

$$
\left|\left(\phi(\lambda), \beta_{j}\right)\right|_{w} \leqq|\phi(\lambda)|_{w}^{2}
$$

also,

$$
\left|\left(M_{\mu}^{\prime}(\lambda) w_{j}, w_{j}\right)\right|_{W}=\left|\sqrt{ } M_{\mu}^{\prime}(\lambda)\left(w_{j}\right)\right|_{W}^{2} \leqq\left|\sqrt{ } M_{\mu}^{\prime}(\lambda)\right|_{B}^{2} .
$$

It follows that the integrand on RHS(1) does not exceed

$$
|\phi(\lambda)|_{W}^{2}\left|\sqrt{ } M_{\mu}^{\prime}(\lambda)\right|_{B}^{2}
$$

hence by (ii) the integrals in (1) are finite, as required for (a).

(b) $\forall$ finite $J_{0} \subset J$, we have cf. 9.28(b)

$$
\begin{array}{ll}
\left|\phi(\cdot)-\sum_{j \in J_{0}}\left(\phi(\cdot), w_{j}\right) w_{j}\right|_{M} & \\
& =\int_{\Lambda}\left|\sqrt{ } M_{\mu}^{\prime}(\lambda)\left\{\phi(\lambda)-\sum_{j \in J_{0}}\left(\phi(\lambda), w_{j}\right)_{W} w_{j}\right\}\right|_{W}^{2} \mu(d \lambda) \\
& =\int_{\Lambda} \psi_{J_{0}}(\lambda) \mu(d \lambda), \text { say. }
\end{array}
$$

From (2) it follows that

$$
\forall \lambda \in \Lambda, \quad \psi_{J_{0}}(\lambda) \rightarrow 0, \quad \text { as } J_{0} \rightarrow J .
$$

Also, from (2)

$$
\left|\phi(\lambda)-\sum_{j \in J_{0}}\left(\phi(\lambda), w_{j}\right) w_{j}\right|_{w} \leqq|\phi(\lambda)|_{W}^{2}-\sum_{j \in J_{0}}\left|\left(\phi(\lambda), w_{j}\right)_{W}\right|^{2}
$$

and hence,

$$
\begin{aligned}
0 \leqq \psi_{J_{0}}(\lambda) & \leqq\left|\sqrt{ } M_{\mu}^{\prime}(\lambda)\right|_{B}^{2}\left\{|\phi(\lambda)|_{W}^{2}-\sum_{j \in J_{0}}\left|\left(\phi(\lambda), w_{j}\right)\right|_{W}^{2}\right\} \\
& \leqq\left|\sqrt{ } M_{\mu}^{\prime}(\lambda)\right|_{B}^{2}|\phi(\lambda)|_{W}^{2} .
\end{aligned}
$$

From (4), (6), (ii) and Lebesgue's Thm. on Dominated Convergence, it follows that 


$$
\int_{\Delta} \psi_{J_{0}}(\lambda) \mu(d \lambda) \rightarrow 0, \quad \text { as } J_{0} \rightarrow J
$$

i.e. by (3) that

$$
\phi=\sum_{j \in J_{0}}\left(\phi(\cdot), w_{j}\right)_{W} w_{j} \quad \text { in } \mathfrak{L}_{2, W} .
$$

(c) From (b) and Lemma 10.3(e) \& (c) we get

$$
\begin{aligned}
\int_{\Delta} T(d \lambda) \phi(\lambda) & =\lim _{J_{0} \rightarrow J} \int_{\Delta} T(d \lambda)\left\{\sum_{j \in J_{0}}\left(\phi(\lambda), w_{j}\right)_{W} w_{j}\right\} \\
& =\lim _{J_{0} \rightarrow J} \sum_{j \in J_{0}} \int_{\Lambda} T(d \lambda)\left\{\left(\phi(\lambda), w_{j}\right)_{W} w_{j}\right\} \\
& =\lim _{J_{0} \rightarrow J} \sum_{j \in J_{0}} \int_{\Delta}\left(\phi(\lambda), w_{j}\right)_{W} \xi_{j}(d \lambda) \\
& =\sum_{j \in J} \int_{\Lambda}\left(\phi(\lambda), w_{j}\right)_{W} \xi_{j}(d \lambda),
\end{aligned}
$$

where the penultimate step follows from 10.10 .

The corresponding result for measures subject to (9.16) and (9.20) is as follows:

10.32. ThEOREM. Let

(i) the measures $M \& \mu$ be as in (9.16) \& (9.20),

(ii) $\forall j \in J, \beta_{j}$ be a unit vector in Range $P_{j}$,

(iii) $\forall j \in J \quad \xi_{j}(\cdot)={ }_{d} T(\cdot)\left(\beta_{j}\right)$.

Then

(a) $\forall j \in J, \xi_{j}(\cdot)$ is a $\mathfrak{H C}$-valued, c.a.o.s. measure over $\left(\Lambda, \wp, \mu_{j}\right)$;

(b) $\forall j, k \in J, j \neq k, \quad \delta_{\xi_{j}} \perp \delta_{\xi_{k}}$;

(c) $\forall B \in B \& \forall w \in W, \quad T(B)(w)=\sum_{j \in J}\left(w, \beta_{j}\right)_{W} \xi_{j}(B)$;

(d) $\forall B \in B \& \forall \phi \in \mathfrak{L}_{2, W}$

$$
\int_{B} T(d \lambda) \phi(\lambda)=\sum_{j \in J} \int_{B}\left(\phi(\lambda), \beta_{j}\right)_{W} \xi_{j}(d \lambda) ;
$$

(e) $\forall \phi \in \mathfrak{L}_{2, W}$, the measure $\nu_{\phi \phi}$ defined in 10.20(b) is absolutely continuous with respect to $\mu$, and

$$
\frac{d \nu_{\phi \phi}}{d \mu}(\cdot)=\sum_{j \in J}\left|P_{j}\{\phi(\cdot)\}\right|_{W}^{2} \frac{1}{a_{j}} \chi_{\Lambda j}(\cdot) \in L_{1}(\Lambda, \AA, \mu ; R) .
$$

Proof. (a) is clear from $8.5(a)$, since by (9.16) 


$$
M(\cdot)\left(\beta_{j}\right)=\mu_{j}(\cdot) \beta_{j} \quad \& \text { so } \quad\left(M(\cdot) \beta_{j}, \beta_{j}\right)_{W}=\mu_{j}(\cdot) .
$$

(b) follows, since by (iii) and (1),

$$
\left(\xi_{j}(A), \xi_{k}(B)\right)=\left(M(A \cap B) \beta_{j}, \beta_{k}\right)_{W}=\mu_{j}(A \cap B)\left(\beta_{j}, \beta_{k}\right)_{W},
$$

and therefore $\xi_{j}(A) \perp \xi_{k}(B)$ for $j \neq k$.

(c) By (ii), $\left(\beta_{j}, j \in J\right)$ is an o.n. basis for $W$, and $T(B)$ is continuous. Hence

$$
T(B) w=T(B)\left\{\sum_{j \in J}\left(w, \beta_{j}\right) \beta_{j}\right\}=\sum_{k \in J}\left(w, \beta_{j}\right) \xi_{j}(B) .
$$

(d) Let $\phi \in \mathfrak{L}_{2, W}$. We first assert that

$$
\sum_{j=1}^{n}\left(\phi(\cdot), \beta_{j}\right)_{W} \beta_{j} \rightarrow \phi(\cdot) \quad \text { in } \mathfrak{L}_{2, W}, \quad \text { as } n \rightarrow \infty .
$$

For denote the sum on the left by $S_{n}(\cdot)$. Then by 9.29 (b)

$$
\begin{aligned}
\left|\phi(\cdot)-S_{n}(\cdot)\right|_{M}^{2} & =\sum_{j \in J} \int_{\Lambda}\left|\left(\phi(\lambda)-S_{n}(\lambda), \beta_{j}\right)_{W}\right|^{2} \mu_{j}(d \lambda) \\
& =\sum_{j>n} \int_{\Lambda}\left|\left(\phi(\lambda), \beta_{j}\right)_{W}\right|^{2} \mu_{j}(d \lambda) .
\end{aligned}
$$

The RHS $\rightarrow 0$, as $n \rightarrow \infty$, since by 9.29 (b)

$$
\sum_{j \in J} \int_{\Lambda}\left|\left(\phi(\lambda), \beta_{j}\right)_{W}\right|^{2} \mu_{j}(d \lambda)=|\phi|_{M}^{2}<\infty .
$$

Thus (2).

Now let $\phi \in \mathfrak{L}_{2, W}$ and $B \in ß$. Then by (2)

$$
\sum_{j=1}^{n}\left(\phi(\cdot) \chi_{B}(\cdot), \beta_{j}\right)_{W} \beta_{j} \rightarrow \phi(\cdot) \chi_{B}(\cdot) \quad \text { in } \mathscr{L}_{2, W}, \quad \text { as } n \rightarrow \infty .
$$

It follows from $10.3(\mathrm{e})$ and 10.10 that

$$
\begin{aligned}
\int_{B} T(d \lambda) \phi(\lambda) & =\lim _{n \rightarrow \infty} \int_{\Lambda} T(d \lambda)\left\{\sum_{j=1}^{n}\left(\phi(\lambda) \chi_{B}(\lambda), \beta_{j}\right)_{W} \beta_{j}\right\} \\
& =\lim _{n \rightarrow \infty} \sum_{j=1}^{n} \int_{B}\left(\phi(\lambda), \beta_{j}\right)_{W} \xi_{j}(d \lambda) \\
& =\sum_{j \in J} \int_{B}\left(\phi(\lambda), \beta_{j}\right)_{W} \xi_{j}(d \lambda) .
\end{aligned}
$$


(e) Let $\phi \in \mathfrak{L}_{2, W}$ and $B \in \Theta$. Then by $10.20(\mathrm{~b})$, (d), (b) and the Pythagorean identity

$$
\begin{array}{rlr}
\nu_{\phi \phi}(B) & =\left|\int_{B} T(d \lambda) \phi(\lambda)\right|_{3 C}^{2} \\
& =\sum_{j \in J}\left|\int_{B}\left(\phi(\lambda), \beta_{j}\right)_{W} \xi_{j}(d \lambda)\right|_{3 c}^{2} \\
& =\sum_{j \in J} \int_{B}\left|\left(\phi(\lambda), \beta_{j}\right)_{W}\right|^{2} \mu_{j}(d \lambda), & \text { by (a) } \\
& =\sum_{j \in J} \int_{B}\left|\left(\phi(\lambda), \beta_{j}\right)_{W}\right|^{2} \frac{1}{a_{j}} \chi_{\Lambda_{j}}(\lambda) \mu(d \lambda) & \text { by }(9.20) \\
& =\sum_{j \in J} \int_{B} f_{j}(\lambda) \mu(d \lambda), \text { say. }
\end{array}
$$

It follows from B. Levi's Thm. on integration of monotone sequences that

$$
\sum_{j \in J} f_{j}(\cdot) \in L_{1}(\Lambda, \Theta, \mu ; R) \quad \& \quad \nu_{\phi \phi}(B)=\int_{B}\left\{\sum_{j \in J} f_{j}(\lambda)\right\} \mu(d \lambda) .
$$

From this (e) clearly follows.

G. Finally, we shall consider a result needed for vectorial $L_{2}$-transform theory. It shows how any ordinary $L_{2}$-valued, c.a.o.s. measure generates a $W$-to- $\mathfrak{L}_{2, W}$, c.a.q.i. measure in the fashion of Lma. $10.13 .{ }^{31}$

10.33. ThEOREM. Let

(i) $W$ be a complex Hilbert space,

(ii) $\mu$ be a nonnegative, $\sigma$-finite, c.a. measure on a $\sigma$-ring $B$ over a set $\Lambda$,

(iii) $\mathfrak{L}_{2, C}=L_{2}(\Lambda, \Re, \mu ; C), \mathfrak{L}_{2, W}=L_{2}(\Lambda, \Re, \mu I ; W)$,

(iv) $\xi$ be a $\mathfrak{L}_{2, C}$-valued, c.a.o.s. measure over $(Y, \mathcal{F}, \nu), c f .2 .1,2.7$,

(v) $\forall A \in \mathcal{F}_{\nu} \& \forall w \in W,\left\{M_{\xi}(A)\right\}(w)={ }_{d} w \xi_{A}(\cdot)$. Then

(a) $M_{\xi}$ is a W-to- $\mathfrak{L}_{2, W}$, c.a.q.i. measure over $\left(Y, \mathfrak{F}_{\nu}, \nu I\right)$;

(b) $\forall \phi \in \mathfrak{L}_{2}\left(Y, \mathfrak{F}, \nu ;\right.$ C) \& $\forall w \in W, \int_{Y} M_{\xi}(d y)\{w \phi(y)\}=w \int_{Y} \phi(y) \xi_{d y}$;

(c) $M_{\xi}$ is $W$-to- $\mathfrak{L}_{2, W}$ basic, provided $\xi$ is $\mathfrak{L}_{2, C}$ basic;

(d) $\forall \phi \in \mathscr{L}_{2, W} \& \forall A \in \mathcal{F}_{\nu}, \overline{\xi_{A}(\cdot)} \phi(\cdot) \in L_{1}(\Lambda, B, \mu ; W), \quad \&$

${ }^{31}$ But 10.13 is not a corollary of 10.33 , for in the latter we are imposing the restriction $M(\cdot)=\mu(\cdot) I$. This severe restriction of course makes $\mathfrak{L}_{2, W}$ a Hilbert space. 


$$
\left\{M_{\xi}(A)\right\}^{*}(\phi)=\int_{\Delta} \overline{\xi_{A}(\lambda)} \phi(\lambda) \mu(d \lambda),
$$

where the last is a $W$-valued Bochner integral.

Proof. (a) Let $A, B \in F_{\nu}$ and $w, w^{\prime} \in W$. Then by (iv) $\xi_{A}(\cdot) \in \mathfrak{L}_{2, C}$ and so by $9.25(\mathrm{~h}) w \xi_{A}(\cdot) \in \mathfrak{L}_{2, W}$; also obviously

$$
M_{\xi}(A) \text { is a continuous lin. oper. on } W \text { to } \mathfrak{L}_{2, W} \text {. }
$$

Next, since $\mathscr{L}_{2, W}$ is as in (iii),

$$
\begin{aligned}
\left(\left\{M_{\xi}(A)\right\}(w),\left\{M_{\xi}(B)\right\}\left(w^{\prime}\right)\right)_{M} & =\left(w \xi_{A}(\cdot), w^{\prime} \xi_{B}(\cdot)\right)_{M} \\
& =\int_{\Lambda}\left(w \xi_{A}(\lambda), w^{\prime} \xi_{B}(\lambda)\right)_{W} \mu(d \lambda) \\
& =\left(w, w^{\prime}\right)_{W}\left(\xi_{A}, \xi_{B}\right)_{\mathfrak{S}_{2}, C} \\
& =\left(w, w^{\prime}\right)_{W} \nu(A \cap B), \quad \text { (by (iv)) }
\end{aligned}
$$

By (1) and (2) we have (a).

(b) Let $w \in W$. Then $\forall A \in \mathcal{F}_{\nu}, w \xi_{A} \in \mathfrak{L}_{2, W}$ and from (iv) we see at once that

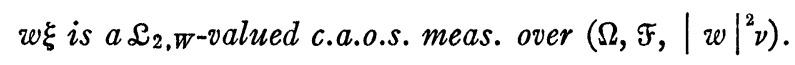

Also, by Triv.10.10,

(4) $\forall \phi \in L_{2}(Y, \mathcal{F}, \nu ; C), \quad \int_{Y} M_{\xi}(d y)\{w \phi(y)\}=\int_{\Lambda} \phi(y)\left\{w \xi_{d \nu}\right\}$.

Now if $\phi_{n}$ is $\Im_{\nu}$-simple, say $\phi_{n}=\sum_{1}^{r} c_{k} \chi_{A_{k}}, A_{k} \in F_{\nu}$, then cf. [30, 5.4]

$$
\int_{Y} \phi_{n}(y)\left\{w \xi_{d y}\right\}=\sum_{1}^{r} c_{k}\left\{w \xi_{A_{k}}\right\}=w \int_{Y} \phi(y) \xi_{d y} .
$$

Next let

$$
\phi=\lim _{n \rightarrow \infty} \phi_{n} \text { in } L_{2}(Y, \mathcal{F}, \nu ; C),
$$

where $\phi_{n}$ are $\mathscr{F}_{\nu}$-simple. Then obviously

$$
\boldsymbol{\phi}=\lim _{n \rightarrow \infty} \phi_{n} \text { in } L_{2}\left(Y, F,|w|^{2} \nu, \mathbf{C}\right) .
$$

Hence

$$
\begin{aligned}
\int_{\Lambda} \phi(y)\left\{w \xi_{d y}\right\} & =\lim _{n \rightarrow \infty} \int_{Y} \phi_{n}(y)\left\{w \xi_{d y}\right\} & & \text { by (7) \& (3) } \\
= & \lim _{n \rightarrow \infty} w \int_{Y} \phi_{n}(y) \xi_{d y} & & \text { by (5) } \\
& =w \int_{Y} \phi(y) \xi_{d y} & & \text { by (6) \& (iv). }
\end{aligned}
$$


Combining (4) and (8) we have (b).

(c) Given that $\S_{\xi}=\mathfrak{L}_{2, C}$, we have to show that $\S_{M_{\xi}}=\mathfrak{L}_{2, w}$. But by Lma. 10.13(a)

$$
\begin{aligned}
\mathfrak{L}_{2, W} & =\mathfrak{S}_{M_{\chi}}=\subseteq\left\{M_{\chi}(B)(W): B \in \mathbb{Q}_{\mu}\right\} \\
& =\Im\left\{w \chi_{B}(\cdot): w \in W \& B \in \mathbb{B}_{\mu}\right\} .
\end{aligned}
$$

Hence it will suffice to show that

$$
\forall w \in W \quad \& \quad \forall B \in \mathcal{B}_{\mu}, \quad w_{B}(\cdot) \in \mathcal{S}_{M_{\xi}} \cdot
$$

Now since $\chi_{B} \in \mathscr{L}_{2, C}=\delta_{\xi}, \quad \exists \phi \in L_{2}(Y, \mathcal{F}, \nu ; C)$ such that

$$
\chi_{B}(\cdot)=\int_{Y} \phi(y) \xi_{d y} .
$$

Hence by (b)

$$
w \chi_{B}(\cdot)=w \int_{Y} \phi(y) \xi_{d y}=\int_{Y} M_{\xi}(d y)\{w \phi(y)\},
$$

and thus (9) holds, cf. Cor. 10.9.

(d) Let $f \in \mathscr{L}_{2, W}$ and $A \in \mathcal{F}_{\nu}$. Then $\xi_{A} \in \mathscr{L}_{2, C}$ and hence

$$
\overline{\xi_{A}(\cdot)} f(\cdot) \in L_{1}(\Lambda, ß, \mu ; W) .
$$

Also, $\forall w \in W$,

$$
\begin{aligned}
\left(\left\{M_{\xi}(A)\right\}^{*}(f), w\right)_{W} & =\left(f,\left\{M_{\xi}(A)\right\}(w)\right)_{\mathfrak{S}_{2, W}}=\left(f, w \xi_{A}\right)_{\mathfrak{S}_{2, W}} \\
& =\int_{\Delta}\left(f(\lambda), w \xi_{A}(\lambda)\right)_{W} \mu(d \lambda), \text { cf. (iii) } \\
& =\int_{\Delta} \overline{\left(\xi_{A}(\lambda) f(\lambda), w\right)_{W} \mu(d \lambda)} \\
& =\left(\int_{\Delta} \overline{\left.\xi_{A}(\lambda) f(\lambda) \mu(d \lambda), w\right)_{W},}\right.
\end{aligned}
$$

where the last step is a consequence of well-known properties of the Bochner integral in Hilbert space. Since (10) holds $\forall w \in W$, we have (d).

The $W$-to- $\mathfrak{L}_{2, W}$, c.a.q.i. measures generated by $\mathfrak{L}_{2, c}$-valued, c.a.o.s. measures are "bi-isometric" in the sense made clear in the following result:

10.34. Corollary. Let

(i)-(iii) be as in the last theorem, 


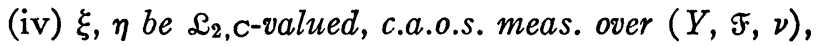

(v) $M_{\xi}, M_{\eta}$ be defined as in the last theorem (v). Then

(a) $\forall A, B \in \mathfrak{F}_{\nu}, \quad M_{\eta}(B) * M_{\xi}(A)=\left(\xi_{A}, \eta_{B}\right)_{\mathcal{L}_{2, \mathrm{C}}}$ Rstr.W $I$

(b) $\forall A, B \in F_{\nu} \& \forall w, w^{\prime} \in W$,

$$
\left(M_{\xi}(A)(w), M_{\eta}(B)\left(w^{\prime}\right)\right)_{M}=\left(\xi_{A}, \eta_{B}\right)_{\mathscr{L}_{2,},}\left(w, w^{\prime}\right)_{W} .
$$

Proof. Let $A, B \in F_{\nu}$ and $w \in W$. Then

$$
\begin{aligned}
M_{\eta}(B) * M_{\xi}(A)(w) & =M_{\eta}(B) *\left\{w \xi_{A}(\cdot)\right\} \\
& =\int_{\Lambda} \overline{\eta_{B}(\lambda)} \cdot w \xi_{A}(\lambda) \mu(d \lambda), \text { by } 10.33(\mathrm{~d}) \\
& =\left\{\int_{\Lambda} \overline{\eta_{B}(\lambda)} \xi_{A}(\lambda) \mu(d \lambda)\right\} \cdot w \\
& =\left(\xi_{A}, \eta_{B}\right)_{\mathcal{L}_{2}, \boldsymbol{C}} \cdot w,
\end{aligned}
$$

where the penultimate step follows from a simple property of the Bochner integral. This proves (a). (b) follows easily from (a).

\section{Theory for locally compact semigroups and groups}

In this section

(i) $\mathfrak{H}_{\mathrm{C}}$ is a Hilbert space and $W$ a separable one, both over $C$;

(ii) $\Lambda$ is an (additive) l.c. ${ }^{32}$ semigroup with neutral element 0 , and $B$ is the family of Borel subsets of $\Lambda$;

(11.1) (iii) $M$ and $\beta_{0}$ are as in (10.1)(iii), where now $\Lambda, B$ are as in (ii), and furthermore $M$ is adequate according to Criterion 9.9;

(iv) $\mathfrak{L}_{2, W}={ }_{d} L_{2}(\Lambda, \Theta, M ; W)$ is thus a Hilbert space;

(v) $T$ is a $W$-to-JC, c.a.q.i. measure over $\left(\Lambda, \Theta_{0}, M\right)$.

It follows of course from (ii) that

$$
\forall \lambda \in \Lambda \& \forall B \in B, \quad \lambda+B \& B+\lambda \in \Theta .
$$

We shall denote by $\tau_{h}, \sigma_{h}$ the right-translation operation and its adjoint on the space of all functions $\phi$ on $\Lambda$, so that

$$
\forall h, \lambda \in \Lambda,\left(\tau_{h} \phi\right)(\lambda)=\phi(\lambda+h),\left(\sigma_{h} \phi\right)(\lambda)= \begin{cases}\phi(\lambda-h), & \lambda \in \Lambda+h, \\ 0, & \lambda \in \Lambda \backslash(\Lambda+h) .\end{cases}
$$

\footnotetext{
82 Not necessarily abelian.
} 
The operation + induces a right-congruence relation $\equiv$ on $B$ defined by: $B \equiv A$ iff $B=A+\lambda$, which brings up questions concerning the relation between the $M$ and $T$ measures of congruent sets. Guided by the usage of the terms "continuous" and "stationary" in the theory of stochastic processes, we shall adopt the following definition:

11.4. Definition. (a) We say that the c.a.q.i. measure T of (11.1) is right-continuous, iff

$$
\forall B \in \Theta_{0} \& \forall \lambda \in \Lambda, \quad B+\lambda \in \Theta_{0} \quad \& \quad \operatorname{sim}_{\lambda \rightarrow 0} T(B+\lambda)=T(B) .
$$

(b) We say that $T$ is right-stationary, iff the associated measure $M$ is right-invariant, i.e.

$\forall B \in \Theta_{0} \& \forall \lambda \in \Lambda, \quad B+\lambda \in \Theta_{0} \quad \& \quad M(B+\lambda)=M(B)$.

Note. In the sequel we shall of ten omit the prefix "right." Thus, continuous, stationary, invariant, etc. will mean right-continuous, right-stationary, right-invariant, etc.

From 8.7 (b) we see at once that

$$
T \text { is continuous } \Leftrightarrow \forall B \in \bigotimes_{0}, \operatorname{sim}_{\lambda \rightarrow 0} M\{(B+\lambda) \triangle B\}=0 .
$$

The condition on the right may be construed as a "continuity condition" on the measure $M$.

In analogy with stochastic processes, continuity and stationarity are together necessary and sufficient for the existence of an isometric shift semigroup for our measure. To show this we need the following lemmas, the proofs of which we shall leave to the reader.

11.6. Lemma. Let the measure $M$ of (11.1)(iii) be "continuous" and invariant, ${ }^{33}$ i.e. $\forall B \in \beta_{0} \& \forall \lambda \in \Lambda$,

$$
B+\lambda \in B_{0}, \quad \operatorname{sim}_{\lambda \rightarrow 0} M\{(B+\lambda) \triangle B\}=0, \quad M(B+\lambda)=M(B) .
$$

Then

(a) $\forall \phi, \psi \in \mathfrak{L}_{2, W} \& \forall \lambda, h \in \Lambda$,

$$
\begin{gathered}
\int_{\Lambda}\left(\sigma_{h} \psi\right)(\lambda)^{*} M(d \lambda)\left(\sigma_{h} \phi\right)(\lambda)=\int_{\Lambda} \psi(\lambda)^{*} M(d \lambda) \phi(\lambda), \\
\left(\sigma_{h} \phi, \sigma_{h} \psi\right)_{M}=(\phi, \psi)_{M} \quad \& \quad\left|\sigma_{h} \phi\right|_{M}=|\phi|_{M}
\end{gathered}
$$

${ }^{33}$ When $\Lambda$ is a 1.c. group invariance implies continuity as we shall show in 11.14(b). But we do not know if this is true for l.c. semigroups in general. 
(b) $\left(\sigma_{\lambda}, \lambda \in \Lambda\right)$ is a strongly continuous semigroup of isometries on the Hilbert space $\mathfrak{L}_{2, W}$ into $\mathfrak{L}_{2, W}$.

11.7. LEMma. Let

(i) $\mathfrak{T}, \mathfrak{K}$ be Hilbert spaces over $\mathbf{C}$,

(ii) $S$ be a function with domain $D \subseteq \mathcal{H C}$ and range $R \subseteq \mathcal{H}$ such that

$$
\forall x, y \in \mathfrak{H}, \quad(S x, S y)_{\Re}=(x, y)_{\Re C}
$$

(iii) $\bar{S}={ }_{d} S(S)$ be the (closed) linear subspace spanned by $S$ in the direct sum $\mathfrak{H C} \times \mathfrak{K}$.

Then $\bar{S}$ is a (s.v.) closed, linear isometry on $\mathfrak{S}(D)$ onto $\mathfrak{S}(R)$.

With the aid of these lemmas we can prove the following analogue of our $[30,7.5]$ and of classical results on stationary stochastic processes, cf. $[20$, p. 55]:

11.8. Theorem (EXISTENCE OF SHIFT semigroup). The following conditions are equivalent:

$(\alpha)$ the measure $T$ is right-continuous and right-stationary,

( $\beta) \exists$ a strongly continuous semigroup $\left(\bar{S}_{\lambda}, \lambda \in \Lambda\right)$ of isometries on $\mathfrak{S}_{T}$ into $S_{T}$ such that

$\forall \lambda \in \Lambda \& \forall B \in B_{0}, \quad B+\lambda \in \mathbb{B}_{0} \& \bar{S}_{\lambda} \circ\{T(B)\}=T(B+\lambda)$.

Proof. We may replace $(\alpha)$ by its equivalent version, $\mathrm{cf}$. (11.5), $\left(\alpha^{\prime}\right)$

$\forall \lambda \in \Lambda \& \forall B \in B_{0}$,

$$
B+\lambda \in \Theta_{0}, \quad \operatorname{sim}_{\lambda \rightarrow 0} M\{(B+\lambda) \triangle B\}=0, \quad M(B+\lambda)=M(B) .
$$

The implication $(\beta) \Rightarrow\left(\alpha^{\prime}\right)$ is obvious. For, let $(\beta)$ hold, $\lambda \in \Lambda$ and $B \in \beta_{0}$. Then $B+\lambda \in \beta_{0}$, and

$$
\operatorname{slim}_{\lambda \rightarrow 0} T(B+\lambda)=\operatorname{sim}_{\lambda \rightarrow 0} \bar{S}_{\lambda} \circ T(B)=T(B),
$$

and hence by Lma.8.7(b),

$$
\operatorname{slim}_{\lambda \rightarrow 0} M\{(B+\lambda) \triangle B\}=0 .
$$

Also, cf. 8.2(ii),

$$
\begin{aligned}
M(B+\lambda) & =T(B+\lambda)^{*} T(B+\lambda)=T(B)^{*} \bar{S}_{\lambda}^{*} \bar{S}_{\lambda} T(B) \\
& =T(B) * T(B)=M(B) .
\end{aligned}
$$

Thus $\left(\alpha^{\prime}\right)$ holds. 
Next let $\left(\alpha^{\prime}\right)$ hold. ${ }^{34}$ For a fixed $\lambda \in \Lambda$ define the operator $S_{\lambda}$ on the linear manifold $T\left(\Re_{0}\right)(W) \subseteq \mathcal{S}_{T} \subseteq \mathfrak{F}$ by

$$
S_{\lambda}\{T(A)(w)\} \underset{d}{=} T(A+\lambda)(w), \quad A \in \bigotimes_{0}, \quad w \in W .
$$

We claim that $S_{\lambda}$, so defined, is a s.v. operator on $T\left(\otimes_{0}\right)(W)$ into itself, not dependent on the choice of $A$ and $w$. To see this, first note that

$$
\forall A, B \in B_{0}, \quad(A \cap B)+\lambda=(A+\lambda) \cap(B+\lambda),
$$

and therefore,

$$
\begin{aligned}
\left(T(A) w, T(B) w^{\prime}\right)_{\Re} & =\left(M(A \cap B) w, w^{\prime}\right)_{W} & & \text { by }(8.3) \\
& \left.=(M\{A \cap B)+\lambda\} w, w^{\prime}\right)_{W} & & \text { by }(\alpha) \\
& =\left(M\{(A+\lambda) \cap(B+\lambda)\}_{w}, w^{\prime}\right)_{W} & & \text { by }(2) \\
& =\left(T(A+\lambda) w, T(B+\lambda) w^{\prime}\right)_{W} & & \text { by }(8.3) .
\end{aligned}
$$

It easily follows from (3) and $\left(\alpha^{\prime}\right)$ that

$$
\left|T(A) w-T(B) w^{\prime}\right|_{\Re C}^{2}=\left|T(A+\lambda) w-T(B+\lambda) w^{\prime}\right|_{\mathscr{F}}^{2} .
$$

Hence $\forall A, B \in B_{0} \& \forall w, w^{\prime} \in W$

$$
T(A) w=T(B) w^{\prime} \Rightarrow T(A+\lambda) w=T(B+\lambda) w^{\prime} .
$$

Thus,

$S_{\lambda}$ is a s.v. operator on $T\left(B_{0}\right)(W)$ into itself.

The equation (3) also shows that $\forall A, B \in \Theta_{0} \& \forall w, w^{\prime} \in W$

$$
\left(S_{\lambda}\{T(A) w\}, S_{\lambda}\left\{T(B) w^{\prime}\right\}\right)_{\mathfrak{F C}}=\left(T(A) w, T(B) w^{\prime}\right)_{\mathfrak{H C}},
$$

i.e.

$$
\forall x, y \in T\left(B_{0}\right)(W) \subseteq \mathfrak{H C}, \quad\left(S_{\lambda}(x), S_{\lambda}\left(x^{\prime}\right)\right)_{\mathfrak{F C}}=\left(x, x^{\prime}\right)_{\mathfrak{F C}}
$$

Now let $\bar{S}_{\lambda}={ }_{d} \subseteq\left(S_{\lambda}\right)$ be the (closed) linear subspace spanned by $S_{\lambda}$ in the direct sum $\mathfrak{H C} \times \mathfrak{H C}$. Then by the last lemma, $\bar{S}_{\lambda}$ is an isometry on $\mathfrak{S}(D)$ onto $\mathfrak{S}(R)$. But by $(4) D=T\left(B_{0}\right)(W) \supseteq \Re$, and so $\mathfrak{S}(R) \subseteq \mathfrak{S}(D)$ $=S_{T}$. Thus,

$$
\forall \lambda \in \Lambda, \quad \bar{S}_{\lambda} \text { is an isometry on } \mathrm{S}_{T} \text { into } \mathrm{S}_{T} .
$$

In view of (6) we can now restate (1) in the form

$$
\forall \lambda \in \Lambda \& \forall A \in B_{0}, \quad \bar{S}_{\lambda} \circ T(A)=T(A+\lambda) \text {. }
$$

\footnotetext{
${ }^{34}$ Our proof now parallels that of [30, Thm.9.5].
} 
Next from (1) and (4) we easily see that

$$
\forall \lambda, \lambda^{\prime} \in \Lambda, \quad S_{\lambda} S_{\lambda^{\prime}}=S_{\lambda+\lambda^{\prime}} .
$$

But since $S_{\lambda}, S_{\lambda^{\prime}}, S_{\lambda+\lambda^{\prime}}$ are single valued and have the same closed domain $S_{T}$, it follows that

$$
\bar{S}_{\lambda+\lambda^{\prime}}=\subseteq\left(S_{\lambda+\lambda^{\prime}}\right)=\subseteq\left(S_{\lambda} \cdot S_{\lambda^{\prime}}\right)=\subseteq\left(S_{\lambda}\right) \cdot \mathfrak{S}\left(S_{\lambda^{\prime}}\right)=\bar{S}_{\lambda} \cdot \bar{S}_{\lambda^{\prime}} .
$$

Finally, let $h \in \Lambda$, and $x \in \delta_{T(\cdot)}$, say $x=\int_{\Lambda} T(d \lambda) \phi(\lambda)$, where $\phi \in \mathfrak{L}_{2, W}$. Then by (6), 10.12, (7) and the Substitution Rule 10.27

$$
\begin{aligned}
\bar{S}_{h}(x) & =\int_{\Lambda} T(d \lambda+h) \phi(\lambda)=\int_{\Lambda+c} T\left(d \lambda^{\prime}\right) \phi\left(\lambda^{\prime}-h\right) \\
& =\int_{\Lambda} T\left(d \lambda^{\prime}\right)\left(\sigma_{h} \phi\right)\left(\lambda^{\prime}\right), \quad \text { by }(11.3) .
\end{aligned}
$$

Hence

$$
\begin{array}{rlrl}
\left|\left(\bar{S}_{\lambda+h}-\bar{S}_{\lambda}\right)(x)\right|_{\Re C}^{2}=\left|\left(S_{h}-I\right)(x)\right|_{\Re C}^{2} & & \text { by }(8) \\
& =\left|\int_{\Lambda} T(d \lambda)\left(\sigma_{h} \phi\right)(\lambda)-\int_{\Lambda} T(d \lambda) \phi(\lambda)\right|_{\Re C}^{2} & & \text { by }(9) \\
& =\left|\sigma_{h}(\phi)-\phi\right|_{M}^{2} & & \text { by } 10.3(\mathrm{~d}) \\
& \rightarrow 0, \text { as } h \rightarrow 0, & & \text { by } 11.6(\mathrm{~b}) .
\end{array}
$$

By (6), (7), (8) \& (10) we have $(\beta)$.

11.9. Definition. For a right-continuous, right-stationary, c.a.q.i. measure $T$, we shall call the $\left(\bar{S}_{\lambda}, \lambda \in \Lambda\right)$ given by $11.8(\beta)$ the right-shift semigroup of $T$.

For continuous, stationary, c.a.q.i. measures we have as announced for the abelian case in $[31,(3.5),(3.6)],{ }^{35}$ the following strengthened version of our Isomorphism Thm.10.8:

11.10. ISOMORPHISM THEOREM (CONTINUOUS, STATIONARY $T$ OVER SEMIGROUP). Let the measure $T$ of $(11.1)(\mathrm{v})$ be right-continuous and right-stationary with shift semigroup $\left(S_{\lambda}, \lambda \in \Lambda\right)$. Then

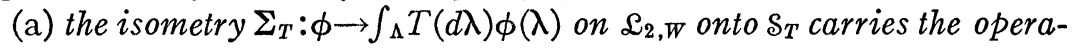
tion $\sigma_{\lambda}$ on $\mathcal{L}_{2, W}$ into the operation $S_{c}$ on $\S_{T}$, i.e.

${ }^{35}$ In $[31,(3.6)]$ Rstr. $s_{T} S_{c}$ should replace $S_{c}$, and $\sigma_{c}$ should replace $\tau_{-c}$. The use of $\tau_{-c}$ is correct provided that $\mathfrak{L}_{2, W}$ is redefined with reference to a larger group as in (11.12) below. Unfortunately, this intended qualification is not stated in [31], except for a special case on p. 618 , footnote. 


$$
\forall \lambda \in \Lambda, \quad S_{\lambda}=\Sigma_{T} \circ \sigma_{\lambda} \circ \Sigma_{T}^{-1} ;
$$

(b) $\forall c \in \Lambda, \forall B \in \mathcal{O}^{\text {loo }} \& \forall \phi \in \mathcal{L}_{2, W}$,

$$
S_{c}\left\{\int_{B} T(d \lambda) \phi(\lambda)\right\}=\int_{B+c} T(d \lambda) \phi(\lambda-c)=\int_{B+c} T(d \lambda)\left(\sigma_{c} \phi\right)(\lambda) .
$$

Proof. (a) Let $c \in \Lambda$. We shall first show that

(A) $S_{c}\left\{\int_{\Lambda} T(d \lambda) \phi(\lambda)\right\}=\int_{\Lambda+c} T(d \lambda) \phi(\lambda-c)=\int_{\Lambda} T(d \lambda)\left\{\sigma_{c} \phi(\lambda)\right\}$.

The second equality in (A) is immediate from the definition of $\sigma_{c} \phi$. Hence we need only show that $\operatorname{LHS}(\mathrm{A})=\operatorname{RHS}(\mathrm{A})$.

Case (i). Let $\phi(\lambda)={ }_{d} \sum_{1}^{r} w_{k} \chi_{A_{k}}, w_{k} \in W \& A_{k} \in ß$. Then by 10.4 and $11.8(\beta)$

$$
\operatorname{LHS}(\mathrm{A})=S_{c}\left\{\sum_{1}^{r} T\left(A_{k}\right) w_{k}\right\}=\sum_{1}^{r} T\left(A_{k}+c\right) w_{k} .
$$

Also, since $\sigma_{c} \phi=\sum_{1}^{r} w_{k} \chi_{A_{k}+c}$, therefore by 10.4

$$
\operatorname{RHS}(\mathrm{A})=\int_{\Lambda} T(d \lambda)\left\{\sum_{1}^{r} w_{k} \chi_{A_{k}+c}(\lambda)\right\}=\sum_{1}^{r} T\left(A_{k}+c\right) w_{k} .
$$

Case (ii). Let $\phi=\lim _{n \rightarrow \infty} \phi_{n}$ in $\mathfrak{L}_{2, W}$, where the $\phi_{n}$ are as in Case (i). Then by the continuity of $S_{c}$ and Case (i),

$$
\begin{aligned}
\operatorname{LHS}(\mathrm{A}) & =\lim _{n \rightarrow \infty} S_{c}\left\{\int_{\Lambda} T(d \lambda) \phi_{n}(\lambda)\right\} \\
& =\lim _{n \rightarrow \infty} \int_{\Lambda} T(d \lambda)\left(\sigma_{c} \phi_{n}\right)(\lambda) .
\end{aligned}
$$

But by Lma.11.6(a),

$$
\left|\sigma_{c} \phi_{n}-\sigma_{c} \phi\right|_{M}=\left|\phi_{n}-\phi\right|_{M} \rightarrow 0, \text { as } n \rightarrow \infty,
$$

i.e. $\sigma_{c} \phi=\lim _{n \rightarrow \infty} \sigma_{c} \phi_{n}$ in $\mathfrak{L}_{2, w}$. Hence by $10.3(\mathrm{e})$, RHS(1) $=$ RHS(A), which of course establishes (A).

By (A), $\forall \phi \in \mathfrak{L}_{2, W}$,

$$
S_{c} \circ \Sigma_{T}(\phi)=S_{c}\left\{\int_{\Lambda} T(d \lambda) \phi(\lambda)\right\}=\int_{\Lambda} T(d \lambda)\left(\sigma_{c} \phi\right)=\Sigma_{T} \circ \sigma_{c}(\phi) .
$$

Thus $S_{c} \circ \Sigma_{T}=\Sigma_{T} \circ \sigma_{c}$, as required for (a).

(b) Let $B \in B^{\text {loe }}$. Then 


$$
\begin{array}{rlr}
\operatorname{LHS}(\mathrm{b}) & =S_{c}\left[\int_{\Lambda} T(d \lambda)\left\{\phi(\lambda) \chi_{B}(\lambda)\right\}\right] & \text { by (A) } \\
& =\int_{\Lambda+c} T(d \lambda)\left\{\phi(\lambda-c) \chi_{B}(\lambda-c)\right\}, \\
& =\int_{\Lambda+c} T(d \lambda)\left\{\phi(\lambda-c) \chi_{B+c}(\lambda)\right\} & \\
& =\int_{B+c} T(d \lambda) \phi(\lambda-c), & \text { since } B+c \subseteq \Lambda+c .
\end{array}
$$

This proves the first equality in (b). The second is obvious from the definition of $\sigma_{c}$.

The last theorem yields as a corollary the translational properties of the spatial integrals (10.24) and of the corresponding subspacevalued and projection-valued measures (cf. 10.25):

11.11. CoROLlaRy. Let the measure $T$ of (11.1)(v) be right-stationary with shift semigroup $\left(S_{\lambda}, \lambda \in \Lambda\right)$. Then

(a) $\forall \lambda \in \Lambda \& \forall B \in \mathbb{B}^{\text {loc }}, S_{\lambda}\left\{\int_{B} T(d \lambda)(W)\right\}=\int_{B+\lambda} T(d \lambda)(W)$;

(b) $\operatorname{ml}(\cdot)$ and $Q(\cdot)$ being as in Thm.10.25, we have

$\forall \lambda \in \Lambda \& \forall B \in Q^{\text {loo }}, \quad S_{\lambda}\{\mathscr{T}(B)\}=\operatorname{Ir}(B+c), \quad S_{\lambda} \circ Q(B) \circ S_{\lambda}^{*}=Q(B+\lambda)$.

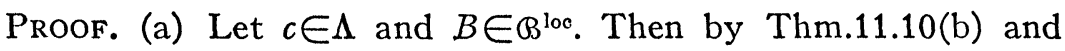
(10.24), $\forall \phi \in \mathfrak{L}_{2, W}$

$$
S_{c}\left\{\int_{B} T(d \lambda) \phi(\lambda)\right\}=\int_{B+c} T(d \lambda)\left(\sigma_{c} \phi\right)(\lambda) \in \int_{B+c} T(d \lambda)(W) .
$$

Hence

$$
S_{c}\left\{\int_{B} T(d \lambda)(W)\right\} \subseteq \int_{B+c} T(d \lambda)(W) .
$$

But obviously $\forall \psi \in \mathfrak{L}_{2, W}, \sigma_{c} \tau_{c}(\psi)=\chi_{\Lambda+c}(\cdot) \psi(\cdot)$, and so by Thm. $11.10(\mathrm{~b})$

$$
\int_{B+c} T(d \lambda) \psi(\lambda)=\int_{B+c} T(d \lambda)\left(\sigma_{c} \tau_{c} \psi\right)(\lambda)=S_{c}\left\{\int_{B} T(d \lambda)\left(\tau_{c} \psi\right)(\lambda)\right\} .
$$

Hence

$$
\int_{B+c} T(d \lambda)(W) \subseteq S_{c}\left\{\int_{B} T(d \lambda)(W)\right\}
$$


By (1) and (2) we have (a). (b) follows trivially.

So far we have not assumed that the semigroup $\Lambda$ is part of a larger group. Suppose that our semigroup, now denoted by $\Lambda_{0}$, is a subsemigroup of an 1.c. group $\Lambda$, and that the measure $M$ is defined as in (11.1)(iii) for the group $\Lambda$ and its Borel family $\leftrightarrow$. Then $\mathfrak{L}_{2, W}=d$ $L_{2}(\Lambda, \Theta, M ; W)$ is a Hilbert space. Denoting by $\AA_{0}, M_{0}$ the restrictions of $B$ and $M$ to $\Lambda_{0}$, it is now convenient to redefine $\mathscr{L}_{2, W}^{0}={ }_{d}$ $L_{2}\left(\Lambda_{0}, \bigotimes_{0}, M_{0} ; W\right)$ by

$$
\mathfrak{L}_{2, W}^{0}=\left\{\phi: \phi \in \mathfrak{L}_{2, W} \& \phi \text { vanishes a.e. } \mu \text { on } \Lambda \backslash \Lambda_{0}\right\} \text {. }
$$

With this interpretation, $\mathfrak{L}_{2, W}^{0}$ is a subspace of $\mathfrak{L}_{2, W}$, and it is clear that

$$
\forall \lambda \in \Lambda_{0} \& \forall \phi \in \mathfrak{L}_{2, W}^{0}, \quad \sigma_{\lambda}(\phi)=\tau_{-\lambda}(\phi) .
$$

Consequently, for continuous, stationary measures the equation in (11.10)(a) can be written

$$
\forall \lambda \in \Lambda_{0}, \quad S_{\lambda}=\Sigma_{T} \circ \tau_{-\lambda} \circ \Sigma_{T}^{-1} .
$$

The correspondence $\Sigma_{T}^{-1}$ may therefore be termed a right-translational representation of the semigroup $\left(S_{\lambda}, \lambda \in \Lambda_{0}\right)$.

The rest of this section is devoted to the simplifications which accrue in the results $11.8,11.10,11.11$ when the semigroup $\Lambda$ is actually a l.c. group. These stem from the fact that an operatorvalued measure which is right-invariant over the entire group $\Lambda$ is a constant operator multiple of the right-Haar measure:

11.14. LEMMA. Let

(i) $\Lambda$ be a l.c. group with right-Haar measure $m$,

(ii) the measure $M$ of (11.1)(iii) be right-invariant, i.e.

$$
\forall \lambda \in \Lambda \& \forall B \in \bigotimes_{0}, \quad B+\lambda \in \Theta_{0} \quad \& \quad M(B+\lambda)=M(B) .
$$

\section{Then}

(a) $\exists$ a nonnegative hermitian operator $H_{0}$ on $W$ to $W$ such that $M(\cdot)=m(\cdot) H_{0}$ on $B_{0}$;

(b) $M$ is "continuous," i.e. $\forall B \in B_{0}, \operatorname{slim}_{\lambda \rightarrow 0} M\{(B+\lambda) \triangle B\}=0$; in fact $\forall B \in B_{0}, \lim _{\lambda \rightarrow 0}|M\{(B+\lambda) \triangle B\}|_{B}=0$.

Proof. (a) Let $w, w^{\prime} \in W$, and

$$
\mu_{w w^{\prime}}(\cdot)=\left(M(\cdot) w, w^{\prime}\right)_{W} \quad \text { on } \bigotimes_{0} .
$$

Then $\mu_{w w^{\prime}}$ is a complex-valued c.a. measure on $\Theta_{0}$, and by (ii) 


$$
\forall \lambda \in \Lambda \& \forall B \in B_{0}, \quad \mu_{w w^{\prime}}(B+\lambda)=\mu_{w w^{\prime}}(B) .
$$

It follows that this equation will also hold for the Hahn extension of $\mu_{w w^{\prime}}$ for all $B$ in the domain of this extension. Hence by the uniqueness of right-Haar measure up to a multiplicative constant,

$$
\mu_{w w^{\prime}}(\cdot)=c_{w w^{\prime}} \cdot m(\cdot) \text { on } \Theta_{0}, \quad \text { where } \quad c_{w w^{\prime}}=\text { const. } \in \mathbf{C} .
$$

Now let $A, B \in B_{0}$ be such that $m(A)>0<m(B)$. Then by (1) and (3), $\forall w, w^{\prime} \in W$,

$$
\left(\frac{M(A)}{m(A)}(w), w^{\prime}\right)_{W}=c_{w w^{\prime}}=\left(\frac{M(B)}{m(B)}(w), w^{\prime}\right)_{W} .
$$

Hence

Thus

$$
M(A) / m(A)=M(B) / m(B)=H_{0}, \text { say. }
$$

$$
\forall A \in \mathrm{B}_{0} \ni m(A)>0, \quad M(A)=m(A) \cdot H_{0} .
$$

But by (1) and (3), $m(A)=0 \Rightarrow M(A)=0$, and so (4) holds for all $A \in B_{0}$. Also, since by (11.1)(iii) $M(A)$ is a nonnegative, hermitian operator on $W$ to $W$, so by (4) is $H_{0}$. This proves (a).

(b) By (a), $\forall B \in \Re_{0}$,

$$
|M\{(B+\lambda) \triangle B\}|_{B}=m\{(B+\lambda) \triangle B\}\left|H_{0}\right|_{B} .
$$

But since $m$ is a (regular) Haar measure, the function $f_{B}$ defined by

$$
f_{B}(\lambda) \underset{d}{=} m\{(B+\lambda) \triangle B\}, \quad \lambda \in \Lambda
$$

is continuous on $\Lambda$, cf. Halmos $\left[13\right.$, p. 266, Thm.A], and so $f_{B}(\lambda)$ $\rightarrow m(B \triangle B)=0$, as $\lambda \rightarrow 0$. Hence (b) follows from (5).

With the aid of this lemma we obtain the following result for groups corresponding to Thm.11.8 for semigroups:

11.15. ThEOREM (EXISTENCE OF SHIFT GROUP). Let $\Lambda$ be a l.c. group with right-Haar measure $m$. Then the following conditions are equivalent:

( $\alpha$ ) the measure $T$ is right-stationary,

$\left(\alpha^{\prime}\right)$ the measure $M$ of 11.1 (iii) is $m(\cdot) H_{0}$, and so $B_{0}=\Theta_{m}$, where $H_{0}$ is a nonnegative, hermitian operator on $W$ to $W$,

$\left(\alpha^{\prime \prime}\right)$ the measure $T$ is right-continuous and right-stationary,

$(\beta) \quad \exists$ a strongly continuous group $\left(U_{\lambda}, \lambda \in \Lambda\right)$ of unitary operators on $S_{T}$ onto $S_{T}$ such that

$\forall \lambda \in \Lambda \& \forall B \in B_{m}, \quad B+\lambda \in B_{m} \quad \& \quad U_{\lambda} \circ\{T(B)\}=T(B+\lambda)$. 
Proof. That $(\alpha) \Leftrightarrow\left(\alpha^{\prime}\right)$ is clear from Def.11.4(b) of stationarity and the last lemma (a). That $\left(\alpha^{\prime}\right) \Leftrightarrow\left(\alpha^{\prime \prime}\right)$ follows from the last lemma (b) in view of (11.5).

To see that $\left(\alpha^{\prime \prime}\right) \Leftrightarrow(\beta)$, first note that by Thm.11.8, the condition $\left(\alpha^{\prime \prime}\right)$ is equivalent to the condition $11.8(\beta)$. Hence it only remains to show that the semigroup $\left(\bar{S}_{\lambda}, \lambda \in \Lambda\right)$ of $11.8(\beta)$ is actually a group of unitary operators on $S_{T}$ onto $S_{T}$.

Now let $\lambda \in \Lambda$. Then by $11.8(\beta), \forall B \in B_{0}, S_{\lambda} \circ T(B-\lambda)=T(B)$, and so

$$
\forall B \in \Re_{0} \& \forall w \in W, \quad T(B) w=S_{\lambda}\{T(B-\lambda) w\} \in S_{\lambda}\left(S_{T}\right) .
$$

Hence

$$
S_{\boldsymbol{T}} \underset{d}{=} \subseteq\left\{T(B) w: B \in \beta_{0} \& w \in W\right\} \subseteq S_{\lambda}\left(S_{T}\right) \subseteq S_{T} .
$$

Thus each $S_{\lambda}$ is on $S_{T}$ onto $S_{T}$, and $S_{\lambda}$ being an isometry it follows that each is unitary on $S_{T}$ onto $S_{T}$. It is now obvious that $\left(S_{\lambda}, \lambda \in \Lambda\right)$ is a group.

11.16. Definition. For a right-stationary c.a.q.i. measure $T$, given as in (11.1) but with $\Lambda$ a l.c. group, we shall call the $\left(U_{\lambda}, \lambda \in \Lambda\right)$ given by 11.15 the right shift-group of $T$.

The following analogue for groups of Theorem 11.10 is immediate from 11.10 and (11.13) in which $\Lambda_{0}=\Lambda$ :

11.17. ISOMORPHISM THEOREM (CONTINUOUS, STATIONARY $T$ OVER GROUPS). Let

(i) $\Lambda$ be a l.c. group with right-Haar measure $m$,

(ii) the measure $T$ of $(11.1)(\mathrm{v})$ be right-stationary with shift group $\left(U_{\lambda}, \lambda \in \Lambda\right)$.

Then

(a) the isometry $\Sigma_{T}: \phi \rightarrow \int_{\Lambda} T(d \lambda) \phi(\lambda)$ on $\mathfrak{L}_{2, W}$ onto $S_{T}$ carries the operation $\tau_{-\lambda}$ on $\mathfrak{L}_{2, W^{35}}$ into the operation $J_{\lambda}$ on $S_{T}$, i.e.

$$
\forall \lambda \in \Lambda, \quad U_{\lambda}=\Sigma_{T} \circ \tau_{-\lambda} \circ \Sigma_{T}^{-1} ;
$$

(b) $\forall \lambda \in \Lambda, \forall B \in \mathbb{Q}^{100} \& \forall \phi \in \mathfrak{L}_{2, w}$,

$$
U_{c}\left\{\int_{B} T(d \lambda) \phi(\lambda)\right\}=\int_{B+c} T(d \lambda) \phi(\lambda-c) .
$$

Also quite obvious is the following analogue for groups of Cor. 11.11:

${ }^{85}$ Where now $\mathcal{L}_{2, W}=L_{2}\left(\Lambda, \bigotimes, m(\cdot) H_{0} ; W\right)$, cf. $11.15\left(\alpha^{\prime}\right)$. 
11.18. Corollary. Let (i) \& (ii) be as in 11.17 , (iii) $M(\cdot)$ and $Q(\cdot)$ be the spatial integral subspace-valued measure and spectral measure of $T, c f .10 .25$. Then

(a) $\forall \lambda \in \Lambda \& \forall B \in B^{\text {loc }}$,

$$
\begin{aligned}
& U_{\lambda}\left\{\int_{B} T(d \lambda)(W)\right\}=\int_{B+\lambda} T(d \lambda)(W) ; \\
& U_{\lambda}\{\operatorname{Tr}(B)\}=\operatorname{Tr}(B+\lambda), \\
& U_{\lambda} \circ Q(B) \circ U_{\lambda}^{*}=Q(B+\lambda) .
\end{aligned}
$$

Now suppose that in the last corollary the c.a.q.i. measure $T$ is $W$-to- $\mathfrak{H C}$ basic, so that $\delta_{T}=\mathfrak{H C}$. Then its shift group $\left(U_{\lambda}, \lambda \in \Lambda\right)$ acts on $\mathcal{H}$ onto $\mathcal{H C}$, and its spatial integral spectral measure $Q(\cdot)$ defined on $\Theta^{\text {loc }}$ becomes a spectral measure for $\mathfrak{K}$, and the two are related as in 11.18(b). We also have the result 11.17 (a) connecting $U_{\lambda}$ and $\tau_{-\lambda}$, and of course the more general result 10.25 (c) connecting $Q(B)$ and $M_{x_{B}}$. We have thus proved the following result:

11.19. Corollary. Let

(i) $\Lambda$ be a l.c. group with right-Haar measure $m$,

(ii) the c.a.q.i. measure $T$ of (11.1) be W-to-HC basic, and right-stationary with shift group $\left(U_{\lambda}, \lambda \in \Lambda\right)$,

(iii) $Q(\cdot)$ be the spatial integral spectral measure of $T$ on $\mathbb{B}^{\text {loc }}$. Then

(a) $Q(\cdot)$ is a "system of imprimitivity" for $\left(U_{\lambda}, \lambda \in \Lambda\right)$, i.e.

$$
\forall \lambda \in \Lambda \& \forall B \in B^{\text {loo }}, \quad U_{\lambda} \circ Q(B) \circ U_{\lambda}^{*}=Q(B+\lambda) ;
$$

(b) the correspondence $\Sigma_{T}: \phi \rightarrow \int_{\Delta} T(d \lambda) \phi(\lambda)$ is a unitary operator on $\mathcal{L}_{2, W^{36}}$ onto He such that

$$
\begin{aligned}
\forall \lambda \in \Lambda \quad \& \quad \forall B \in \mathbb{B}^{\mathrm{loc}}, \quad U_{\lambda}=\Sigma_{T \circ \tau_{-t} \circ \Sigma_{T}^{-1}} \\
\& \quad Q(B)=\Sigma_{T} \circ M_{\chi_{B} \circ \Sigma_{T}^{-1} .}
\end{aligned}
$$

11.20. Remark. The term "system of imprimitivity," due originally to Frobenius, was reintroduced in the present context by Mackey, cf. $[25$, p. 50]. His Imprimitivity Theorem for a l.c. group $\Lambda[24$, Thm.1] says in essence that if $Q(\cdot)$ is any such system for a $\left(U_{\lambda}\right.$, $\lambda \in \Lambda$ ), then there exists an isomorphism $\Sigma$ on an $L_{2}$ space over $\Lambda$ onto TC satisfying the intertwining relations given in 11.19(b). In Cor.11.19 we have shown that under the hypotheses (ii) \& (iii) such an "intertwining" isomorphism $\Sigma$ does indeed exist and has the

${ }^{36}$ Where now $\mathfrak{L}_{2, W}=L_{2}\left(\Lambda, ß, m(\cdot) H_{0} ; W\right)$, cf. 11.15( $\left.\alpha^{\prime}\right)$. 
explicit form $\Sigma_{T}$. From this it is clear that to get an explicit, c.a.q.i. measure-theoretic proof of Mackey's Thm., we have only to show that given a system of imprimitivity $Q(\cdot)$ for $\left(U_{\lambda}, \lambda \in \Lambda\right)$, there exists a Hilbert space $W$ and a measure $T$ satisfying 11.19 (ii) \&(iii). The discussion of the italicized assertion must, however, await our explication of spectral representations $(\$ 14)$.

\section{A new approach to representation theory}

Hitherto representation theorems have been proved $a b$ initio. But many of the proofs reveal common features such as the consideration of everywhere dense linear manifolds, the use of the principle of extension by continuity, the decomposition of a space into a sum of orthogonal, invariant subspaces, etc. Now according to Thm.10.14, every isometry between an arbitrary Hilbert space $\mathcal{H C}$ and a Hilbertian or pre-Hilbertian $\mathfrak{L}_{\mathbf{2}, \boldsymbol{W}}$ space of functions with values in any separable Hilbert space $W$ (in which, moreover, we can take any adequate or nearly adequate $W$-to- $W$, nonnegative, hermitian operator-valued measure) is realizable by integration with respect to a $W$-to-JC, c.a.q.i. measure. It follows that a large number of representation theorems can be recast in the same general form by using our integration theory.

This suggests a common approach to such representation theorems based on the following procedure:

12.1 Procedure. (i) From the given data, find the auxiliary Hilbert space $W$, and define a $W$-to-HC, operator-valued set-function $T(\cdot)$ over a suitable space $\Lambda$;

(ii) Show that $T(\cdot)$ is a $W$-to- $\mathcal{H}$, c.a.q.i. measure, and find $S_{T}$; (The Isomorphism Thm.10.8 then immediately yields a unitary operator $\Sigma_{T}$ on $\mathfrak{L}_{2, W}$ onto $S_{T}$.)

(iii) Use one or more of the results 10.17, 10.18, 10.30- to get the adjoint $\Sigma_{T}^{*}$, which is the desired representation;

(iv) If the space $\Lambda$ is a l.c. semigroup or group, and the measure $M$ is adequate, employ the results of $\$ 11$.

The utility of this approach will depend of course on the ease with which steps (i)-(iv) can be accomplished. Assuming its feasibility, the approach has the merits of explicitness and systematization cited in $\$ 3$ for the case $q=1$.

Steps (i) and (ii) of Procedure 11.1 present no difficulties when the representation problem is to extend a classical unitary transform between two $L_{2}$-spaces to one between the corresponding spaces of vector-valued functions. Suppose that $\Sigma$ is a unitary transform on $L_{2}$ 
onto $\hat{L}_{2}$ defined explicitly in terms of a $\hat{L}_{2}$-basic, c.a.o.s. measure $\eta$ according to Procedure 3.1. We would then accept a unitary transform $\Sigma$ between the corresponding spaces $L_{2, W}, \hat{L}_{2, W}$ of functions with values in the Hilbert space $W$ as a legitimate extension of $\Sigma$, provided that it meets the requirement:

$$
\forall \phi \in L_{2} \quad \& \quad \forall w \in W, \quad \boldsymbol{\Sigma}(w \phi)=w \Sigma(\phi) .
$$

Our Theorem.10.33(b) shows at once that the choices $T(\cdot)={ }_{d} M_{\eta}$ and $\boldsymbol{\Sigma}={ }_{d} \Sigma_{T}$ will fulfill this requirement. Indeed, the other parts of Theorem.10.33 and Corollary.10.34 enable us to derive readily the explicit theory for $\boldsymbol{\Sigma}$ from the corresponding (known) theory for $\boldsymbol{\Sigma}$.

Rather than justify these remarks in general terms, we shall illustrate them by considering in detail the vectorial Fourier-Plancherel transform for 1.c.a. groups (\$13).

In other types of representation problems step (i) of Procedure 12.1 may itself present difficulties: the definitions of the appropriate Hilbert space $W$ and c.a.q.i. measure $T(\cdot)$ may not be obvious and may require a more or less deep analysis of the data. We shall illustrate this by considering spectral representations, in particular Stone's Theorem on the unitary representation of a 1.c.a. group ( $\$ 14$ ), and Cooper's Theorem on the isometric representation of the semigroup $[0, \infty)(\$ 15)$.

\section{The Fourier-Plancherel transform for vectorial functions}

As in $\$ 6$, let $X$ be an (additive) locally compact abelian group with Borel family $B$ and Haar measure $\mu$, and let $\hat{X}$ be the (multiplicative) character group of $X$ with Borel family $\hat{B}$ and dual Haar measure $\hat{\mu}$. Let $B_{0}, \hat{B}_{0}$ be the $\delta$-rings consisting of Borel sets of finite Haar measure. Let further,

(i) $W$ be a separable Hilbert space over $\mathbf{C}$;

(ii) $\mathfrak{L}_{2, W}=L_{2}(X, \Theta, \mu ; W), \hat{\mathfrak{L}}_{2, W}=L_{2}(\hat{X}, \hat{\beta}, \hat{\mu} ; W)$;

(iii) $\mathfrak{L}_{2, C}=L_{2}(X, \Theta, \mu ; C), \hat{\mathfrak{L}}_{2, C}=L_{2}(\hat{X}, \hat{\mathcal{B}}, \hat{\mu} ; C)$;

(iv) $\xi \& \eta$ be the FP $\mathfrak{L}_{2, C^{-}} \& \hat{\mathfrak{L}}_{2, C}$-basic c.a.o.s. measures over $(\hat{X}, \hat{B}, \hat{\mu}) \&(X, B, \mu)$, respectively, $c f .(6.2), 6.4$.

Next recall the $M$-notation used in 10.13 and $10.32(\mathrm{v})$ :

$$
\begin{array}{lll}
\forall \hat{B} \in \hat{\mathscr{B}}_{0} \quad \& \quad \forall w \in W, & \left\{M_{\xi}(\hat{B})\right\}(w)=w \xi_{\hat{B}}(\cdot), \\
\forall B \in \mathbb{B}_{0} \quad \& \quad \forall w \in W, & \left\{M_{\eta}(B)\right\}(w)=w \eta_{B}(\cdot) .
\end{array}
$$

Our Them.10.33 enables us to derive readily the FP transform 
theory for the spaces $\mathfrak{L}_{2, W}, \hat{\mathfrak{L}}_{2, W}$ from the corresponding theory for $\mathfrak{L}_{2, c}, \hat{\mathcal{L}}_{2, c}$ outlined in $\$ 6$ and given fully in [32]. First, from Theem. $10.33(\mathrm{~b})$ and (6.6), (6.7),

$$
\begin{array}{ll}
\forall f \in \mathfrak{L}_{2, C} \quad \& \quad \forall w \in W, & \int_{X} M_{\eta}(d x)\{w f(x)\}=w \hat{f}(\cdot), \\
\forall g \in \hat{\mathfrak{S}}_{2, C} \quad \& \quad \forall w \in W, \quad \int_{\hat{X}} M_{\xi}(d \alpha)\{w g(\alpha)\}=w \tilde{g}(\cdot),
\end{array}
$$

and this, cf. (12.2), indicates the relevancy of the measures $M_{\xi}, M_{\eta}$ for FP theory. Next, from Thms. 6.4 and 10.33(b),(c) we see at once that

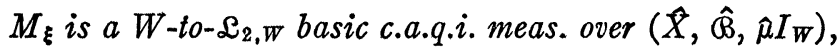

$$
\begin{aligned}
& M_{\eta} \text { is a W-to- } \hat{\mathscr{L}}_{2, W} \text { basic c.a.q.i. meas. over }\left(X, \Theta, \mu I_{W}\right) \text {. }
\end{aligned}
$$

This crucial result when combined with Thm.10.8 and Cor.10.9 in turn shows at once that

(a) The correspondence $\Sigma_{\eta}: f \rightarrow \int_{X} M_{\eta}(d x) f(x)$ is a unitary operator on $\mathfrak{L}_{2, W}$ onto $\hat{\mathfrak{L}}_{2, W}$

(b) The correspondence $\Sigma_{\xi}: g \rightarrow \int_{\hat{X}} M_{\xi}(\alpha) g(\alpha)$ is a unitary operator on $\hat{\mathfrak{L}}_{2, W}$ onto $\mathfrak{L}_{2, W}$.

We now assert the following corollary:

13.6. Corollary. The operators $\Sigma_{\eta}, \Sigma_{\xi}$ of (13.5) are inverses, i.e. $\Sigma_{\xi}=\Sigma_{\eta}^{*}$.

Proof. Grant for a moment that

$$
\forall A \in \hat{C}_{0}, \quad \Sigma_{\eta}^{*} \circ\left\{M_{\chi}(A)\right\}=M_{\xi}(A) .
$$

Then from Lma. 10.13(b), (I) and Lma. 10.12(b), $\forall g \in \hat{\mathcal{L}}_{2, W}$,

$$
\Sigma_{\eta}^{*}(g)=\Sigma_{\eta}^{*}\left\{\int_{\hat{X}} M_{x}(d \alpha) g(\alpha)\right\}=\int_{\hat{X}} M_{\xi}(d \alpha) g(\alpha)=\Sigma_{\xi}(g),
$$

as desired. Hence it only remains to prove (I).

Let $\hat{A} \in \hat{\mathrm{B}}_{0}, w \in W$, and let, cf. (8.11),

$$
\forall B \in B_{0}, \quad \zeta(B)={ }_{d}^{=} \zeta_{w \hat{A}}(B)=M_{\eta}(B)^{*}(w \chi \hat{A}) .
$$

Then on the one hand by Thm.10.17(b)

$$
d \zeta / d \mu=\Sigma_{\eta}^{*}(w \chi \hat{A})=\left\{\Sigma_{\eta}^{*} \circ M_{\chi}(A)\right\}(w), \quad \text { a.e. }(\mu) .
$$


But on the other hand,

$$
\begin{aligned}
\zeta(B) & =\left\{M_{\eta}(B) * M_{\chi}(\hat{A})\right\}(w)=w\left(\chi \hat{A}, \eta_{B}\right)_{\mathfrak{L}_{2, C}} & & \text { by } 10.33(\mathrm{a}) \\
& =w\left(\xi_{\hat{A}}, \chi_{B}\right)_{\mathcal{L}_{2, C}}=\int_{B} w \xi_{\hat{A}}(x) \mu(d x), & & \text { by }(6.3)
\end{aligned}
$$

and so

$$
d \zeta / d \mu=w \xi_{\hat{A}}(\cdot)={ }_{d} M_{\xi}(A)(w), \quad \text { a.e. }(\mu) .
$$

From (2) and (3) we get (I).

13.7. Definition. The operator $\Sigma_{\eta}$ of (13.5) is called the (direct) FP transformation on $\mathfrak{L}_{2, W}$ onto $\hat{\mathfrak{L}}_{2, W}$. We call $\Sigma_{\eta}(f)$ the direct $F \boldsymbol{P}$ transform of $f$ in $\mathcal{L}_{2, W}$ and denote it by $\hat{f}$. We call $\Sigma_{\xi}$, i.e. $\Sigma_{\eta}^{*}$, the (indirect) FP transformation on $\hat{\mathfrak{L}}_{2, W}$ onto $\hat{\mathfrak{L}}_{2, W}$. We call $\Sigma_{\xi}(g)$ the indirect FP transform of $g$ in $\hat{\mathfrak{L}}_{2, W}$, and denote it by $\tilde{g}$.

According to this definition, the FP transforms are given by the explicit formulae:

$$
\begin{aligned}
& \forall f \in \mathfrak{L}_{2, W}, \quad \hat{f}=\int_{\boldsymbol{X}} M_{\eta}(d x) f(x), \\
& \forall g \in \hat{\mathfrak{L}}_{2, W}, \quad \tilde{g}=\int_{\hat{\mathbf{X}}} M_{\xi}(d \alpha) g(\alpha) .
\end{aligned}
$$

The relation between these formulae and (6.6), (6.7) is clear from (13.3):

$$
\begin{array}{lll}
\forall f \in \mathfrak{L}_{2, C} \quad \& \quad \forall w \in W, & \{w f(\cdot)\}^{\wedge}=w \hat{f}(\cdot), \\
\forall g \in \hat{\mathfrak{S}}_{2, C} \quad \& \quad \forall w \in W, & \{w g(\cdot)\}^{\sim}=w \tilde{g}(\cdot) .
\end{array}
$$

From the unitarity of the transformation $\Sigma_{\eta}$ we get at once the Parseval and Bessel identities:

$$
\forall f, g \in \mathfrak{L}_{2, W}(f, g)_{\mathfrak{L}_{2, W}}=(\hat{f}, \hat{g}) \hat{\mathfrak{L}}_{2, W}, \quad|f|_{\mathfrak{L}_{2, W}}=|\hat{f}| \hat{\mathfrak{L}}_{2, W}
$$

The first of these, expanded out, asserts that

$$
\int_{X}(f(x), g(x))_{W} \mu(d x)=\int_{\hat{\mathbf{X}}}(\hat{f}(\alpha), \hat{\mathrm{g}}(\alpha))_{W} \hat{\mu}(d \alpha) .
$$

The following analogous result for $f$ in $\mathfrak{L}_{2, W}$ but $g$ in $\mathfrak{L}_{2, C}$ is proved first for $\beta_{0}$-simple $f$ by using (13.9), and then for any $f$ in $\mathcal{L}_{2, W}$ by the usual limiting argument: 


$$
\begin{gathered}
\forall f \in \mathfrak{L}_{2, W} \quad \& \quad \forall g \in \mathfrak{L}_{2, C}, \quad f(\cdot) \overline{g(\cdot)} \in \mathfrak{L}_{1, W} \quad \& \\
\int_{X} f(x) \overline{g(x)} \mu(d x)=\int_{\hat{\boldsymbol{X}}} \hat{f}(\alpha) \overline{\hat{g}(\alpha)} \hat{\mu}(d \alpha) .
\end{gathered}
$$

From (13.8) a routine derivation using the Substitution Rule 10.28, the shift property $[32,(4.2)]$ of the FP measures and Lma. 10.12 yields the corresponding property of the FP transforms, viz.

13.12. Triviality (Shift PRoperty).

$$
\begin{array}{llll}
\forall f \in \mathcal{L}_{2, W} & \& & \forall x \in X, & \left(\tau_{x} f\right)^{\wedge}=[x, \cdot] \hat{f}(\cdot), \\
\forall g \in \hat{\mathcal{L}}_{2, W} \quad \& \quad \forall \alpha \in \hat{X}, & \left(\tau_{\alpha} g\right)^{\sim}=\overline{[\cdot, \alpha]} g(\cdot),
\end{array}
$$

where $\tau_{x}, \tau_{\alpha}$ are the translation operators on $\mathfrak{L}_{2, W}, \hat{\mathfrak{L}}_{2, w} \cdot{ }^{37}$ Briefly,

$\forall x \in X \& \forall \alpha \in \hat{X}, \quad \tau_{x}=\Sigma_{\xi} \circ M_{[x, \cdot]} \circ \Sigma_{\eta}, \quad \tau_{\alpha}=\Sigma_{\eta} \circ M_{\overline{[\cdot, \alpha]}} \circ \Sigma_{\xi}$.

As in the scalar case (cf. [32, (5.8)]), we have explicit differential formulae for the FP transforms:

13.13. Corollary (Differential formulae).

(a) Let $\forall f \in \mathscr{L}_{2, W} \& \forall \hat{B} \in \hat{\beta}_{0}, \zeta_{f}(\hat{B})={ }_{d} M_{\xi}(\hat{B})^{*}(f)$; then $d \zeta_{f} / d \hat{\mu}=\hat{f}$ a.e. $(\hat{\mu})$.

(b) Let $\forall g \in \hat{\mathcal{L}}_{2, W} \& \forall B \in \beta_{0}, \zeta_{0}(B)=M_{\eta}(B) *(g)$; then $d \zeta_{0} / d \mu=\tilde{g}$ a.e. $(\mu)$.

Proof. (a) Since $f=\hat{f}$, therefore from (13.8) and Thm.10.16(a),

$$
\zeta_{f}(\hat{B})=M_{\xi}(\hat{B})^{*}\left\{\int_{\hat{\mathbf{X}}} M_{\xi}(d \alpha) \hat{f}(\alpha)\right\}=\int_{\hat{\mathrm{B}}} \hat{f}(\alpha) \hat{\mu}(d \alpha),
$$

from which (a) is immediate. (b) is proved similarly.

Next suppose that the groups $X, \hat{X}$ have the Lebesgue property, i.e. $(X, \Theta)$ and $(\hat{X}, \hat{Q})$ are Besicovitch spaces with respect to the Haar measures $\mu, \hat{\mu}$ (cf. 2.5). Then the equality in 10.33(d) shows that the differential formulae in $\mathbf{1 3 . 1 3}$ take on the form:

$$
\begin{aligned}
& \forall f \in \mathfrak{L}_{\mathbf{2}, \boldsymbol{w}}, \quad \hat{f}(\alpha)=\lim _{\hat{\hat{N}}_{\alpha} \rightarrow \alpha} \frac{1}{\hat{\mu}\left(\hat{N}_{\alpha}\right)} \int_{\boldsymbol{X}} \overline{\xi_{\hat{N}_{\alpha}}(x)} f(x) \mu(d x), \quad \text { a.e. }(\hat{\mu}), \\
& \forall g \in \hat{\mathfrak{s}}_{2, W}, \quad \tilde{g}(x)=\lim _{N_{x} \rightarrow x} \frac{1}{\mu\left(N_{x}\right)} \int_{\hat{\ell}} \overline{\eta_{N_{x}}(\alpha)} g(\alpha) \hat{\mu}(d \alpha), \text { a.e. }(\mu) .
\end{aligned}
$$

"I.e., $\forall f \in \mathcal{L}_{2, W} \& \forall y \in X, \quad\left(\tau_{x} f\right)(y)=d f(y+x)$;

$\forall g \in \hat{\mathscr{L}}_{2, W} \& \forall \beta \in \hat{X}, \quad\left(\tau_{a} g\right)(x)={ }_{d} g(\beta \alpha)$. 
It is also easy to show that for any 1.c.a. group $X$, the FP transform is the $\mathscr{L}_{2, W}$-extension of the $\mathfrak{L}_{1, W}$-Fourier transform, the latter being defined in the obvious way as a Bochner integral:

13.15. Corollary. (a) If $f \in L_{1} \cap L_{2}(X, \Theta, \mu ; W)$, then

$$
f(\alpha)=\int_{X} f(x) \overline{[x, \alpha]} \mu(d x), \quad \text { a.e. } \hat{\mu} \text { on } \hat{X} \text {. }
$$

(b) If $g \in L_{1} \cap L_{2}(\hat{X}, \hat{\leftrightarrow}, \hat{\mu} ; W)$, then

$$
\tilde{g}(x)=\int_{\hat{X}} g(\alpha)[x, \alpha] \hat{\mu}(d \alpha), \quad \text { a.e. } \mu \text { on } X .
$$

Proof. (a) Let $f \in L_{1} \cap L_{2}(X, \leftrightarrow, \mu ; W)=\mathfrak{L}_{1, W} \cap \mathfrak{L}_{2, W}$ and let

$$
\forall \hat{B} \in \hat{\mathbb{B}}_{0}, \quad \zeta_{f}(\hat{B})=M_{\xi}(B)^{*}(f) .
$$

Then, in view of Cor.13.13(a), we need only show that

$$
\left(d \zeta_{f} / d \hat{\mu}\right)(\alpha)=\int_{\boldsymbol{X}} f(x) \overline{[x, \alpha]} \mu(d x) .
$$

Now by Thm.10.33(d) and (6.2)

(1) $\zeta_{f}(\hat{B})=\int_{X} \overline{\xi_{\hat{B}}(x)} f(x) \mu(d x)=\int_{X}\left\{\int_{\widehat{B}} \overline{[x, \alpha]} \hat{\mu}(d \alpha)\right\} f(x) \mu(d x)$.

But since $g \in \mathscr{L}_{1, W},[\cdot,-]$ is bounded, and $\hat{\mu}(\hat{B})<\infty$, it easily follows that

$$
[\cdot,-] f(\cdot) \in L_{1}(X \times B, \sigma(\Theta \hat{X} \leftrightarrow), \mu \times \hat{\mu} ; W) .
$$

Hence Fubini's Theorem for Bochner integrals can be applied, and this yields

$$
\zeta_{f}(B)=\int_{\hat{B}}\left\{\int_{X} \overline{[x, \alpha]} f(x) \mu(d x)\right\} \hat{\mu}(d \alpha),
$$

from which (I) is immediate.

(b) is proved similarly.

The last corollary in turn yields for $\sigma$-compact $X$ a limiting expression for the FP transform reminiscent of the classical case:

13.16. Corollary. Let $X$ be $\sigma$-compact. Then

$$
\forall f \in \mathfrak{L}_{2, W}, \quad \hat{f}=\lim _{n \rightarrow \infty} \int_{C_{n}} f(x) \overline{[x, \cdot]} \mu(d x)
$$


where $\left(C_{n}\right)_{1}^{\infty}$ is any sequence of precompact sets in $B$ such that $C_{n} \subseteq C_{n+1}$ and $\cup_{1}^{\infty} C_{n}=X$.

Proof. Let $f \in \mathfrak{L}_{2, W}$. Then $f \chi_{c_{n}} \in \mathfrak{L}_{2, W}$, and therefore by (13.8)

$$
\begin{aligned}
\left(f \chi_{C_{n}}\right)^{\wedge} & =\int_{X} M_{\eta}(d x)\left\{f(x) \chi_{C_{n}}(x)\right\}=\int_{C_{n}} M_{\eta}(d x) f(x) \\
& \rightarrow \int_{X} M_{\eta}(d x) f(x)=\ddot{f}, \quad \text { as } n \rightarrow \infty .
\end{aligned}
$$

But since the support of $f \chi_{C_{n}}$ is inside the precompact set $C_{n}$, therefore $f \chi_{C_{n}} \in \mathfrak{L}_{2, W} \cap \mathfrak{L}_{1, W}$, and so by the last corollary, for almost all $\alpha(\hat{\mu}$ measure),

$$
\left(f \chi C_{n}\right)^{\wedge}(\alpha)=\int_{X} f(x) \chi_{C_{n}}(x) \overline{[x, \alpha]}_{\mu}(d x)=\int_{C_{n}} f(x) \overline{[x, \alpha]}_{\mu}(d x) .
$$

The desired result is immediate from (1) and (2).

We conclude this section with the enunciation of the Paley-Wiener Theorem for functions with values in $W$. We shall take for granted the notion of the Hardy class $H_{2}\left(\Delta_{+} ; W\right)$ of functions $f_{+}$on $\Delta_{+}$to $W$, where $\Delta_{+} \subseteq C$ is the upper bank of $R$, and the result that every such $f_{+}$has a boundary-value $f$, defined a.e.(Leb.) on $\boldsymbol{R}$ and such that $f \in L_{\mathbf{2}}(R ; W)$.

13.17. Theorem (Paley-Wiener). Let

(i) $L_{2}([0, \infty) ; W)=\left\{f: f \in L_{2}(R ; W) \& f=0\right.$ on $\left.(-\infty, 0)\right\}$,

(ii) $L_{2}^{0+}(R ; W)=\left\{f: f\right.$ is the bndry. value of a $f_{+}$in $\left.H_{2}\left(\Delta_{+} ; W\right)\right\}$. Then $f \in L_{2}([0, \infty) ; W)$ iff $\tilde{f}={ }_{d} \Sigma_{\xi}(f) \in L_{2}^{0+}(R ; W)$.

\section{Spectral representations}

This section has three parts A, B, C. In part A we shall review how Stone's Theorem for a unitary representation $\left(U_{x}, x \in X\right)$ of a 1.c.a. group $X$ can be treated from the standpoint expressed in $\S 1$ by deriving it from the Krein-Milman Theorem. Stone's Theorem yields a spectral measure $E(\cdot)$ for such unitary groups and hence also for their infinitesimal generators, i.e. selfadjoint operators. The problem of representing such groups and operators by multiplication operators on $L_{2}$ spaces is thus reduced to that of similarly representing the measure $E(\cdot)$. In part B (Thm. 14.12) we shall obtain an explicit representation for $E(\cdot)$ of this sort by following Procedure 12.1. In part $\mathrm{C}$ we shall deduce from this result explicit representations of the same sort for unitary groups and selfadjoint operators. 
A. Let $X$ be a locally compact abelian group. It is known that the continuous characters of $X$ are extreme points of the (weak* closed) convex set $K$ of complex-valued, continuous, positive definite functions $f$ on $X$ such that $|f|_{\infty} \leqq 1$. This crucial fact shows that Bochner's Theorem for such $f$ is a special case of the Choquet-integral version of the Krein-Milman Theorem, cf. [40, p. 119-]. From Bochner's Theorem we get the following general form of Stone's Theorem in a fairly straightforward way, cf. [41, pp. 385-392]:

14.1. Stone's Theorem (Form I). Let

(i) $X, \hat{X}$ be dual l.c.a. groups, $[x, \alpha]$ be as in $\S 6$, and $\hat{\otimes}$ be the $\sigma$-algebra of all Borel subsets of $\hat{X}$ and their complements, ${ }^{38}$

(ii) $\left(U_{x}, x \in X\right)$ be a strongly continuous group of unitary operators on a (complex) Hilbert space $\mathfrak{F C}$ onto $\mathfrak{H C}$.

Then there exists a unique spectral measure $E(\cdot)$ for $\mathfrak{H C}$ on $\hat{\otimes}$ such that

$$
\forall x \in X, \quad U_{x}=\int_{\hat{x}}[x, \alpha] E(d \alpha) .
$$

Since to every selfadjoint operator $H$ from $\mathcal{H C}$ into $\mathcal{H C}$ corresponds to a group $\left(U_{t}, t \in R\right)$ of unitary operators on $\mathcal{H}$ onto $\mathcal{H}$ having $i H$ as infinitesimal generator, the spectral theorem for selfadjoint operators is an easy corollary of the $X=R$ case of 14.1. These theorems are thus provable in a way which accords with the viewpoint advocated in 1.1, and we may use them freely.

We are concerned, however, with Stone's result as a representation theorem, i.e. with a version such as the following:

14.2. Stone's Theorem (form II). Let (i), (ii) be as in 14.1. Then there exists an index set $J$ and indexed families $\left(\mathfrak{H}_{j}, j \in J\right),\left(\mu_{j}, j \in J\right)$, $\left(S_{j}, j \in J\right)$ of subspaces, measures and maps, satisfying:

(1) $\mathfrak{K C}=\sum_{j \in J} \mathfrak{F C}_{j}, \mathfrak{F}_{j} \perp \mathfrak{F}_{k}, j, k \in J, j \neq k$,

(2) $\forall x \in X \& \forall j \in J, U_{x}\left(\mathcal{F C}_{j}\right) \subseteq \mathcal{F C}_{j}$,

(3) $\mu_{j}$ is a bounded, nonnegative c.a. measure on $\hat{\leftrightarrow}$,

(4) $S_{j}$ is a unitary operator on $L_{2}\left(\hat{X}, \hat{\otimes}, \mu_{j} ; C\right)$ onto $\mathfrak{H}_{j}$ such that $\forall x \in X$, $\operatorname{Rstr}_{\cdot{ }_{x_{j}}} U_{x}=S_{j} \circ M_{[x, \cdot]} \circ S_{j}^{-1}$, where $M_{[x, \cdot]}$ is the operation of multiplication by $[x, \cdot]$.

Now Thm. 14.2 can be proved by applying the following theorem to the spectral measure $E(\cdot)$ obtained from Thm. 14.1:

14.3. Theorem (SPeCtral Representation I). Let $E(\cdot)$ be a spec-

${ }^{38}$ Thus, $\hat{B}$ now has a connotation different from what it had in $\S 6$, where it stood for the family of Borel subsets of $\hat{X}$, i.e. the $\sigma$-ring generated by the compact subsets of $\hat{X}$. 
tral measure for $\mathfrak{H C}$ on a $\sigma$-algebra $B$ over a set $\Lambda$. Then $\exists$ an index set $J$ and indexed families $\left(\mathfrak{H}_{j}, j \in J\right),\left(\mu_{j}, j \in J\right),\left(S_{j}, j \in J\right)$ of subspaces, measures and maps, satisfying:

(1) $\mathfrak{H C}=\sum_{j \in J} \mathfrak{H C}_{j}, \quad \mathfrak{K}_{j} \perp \mathfrak{H}_{k}, j, k \in J, j \neq k$,

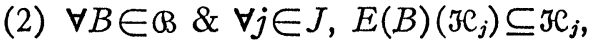

(3) $\mu_{j}$ is a bounded, nonnegative, c.a. measure on $B$,

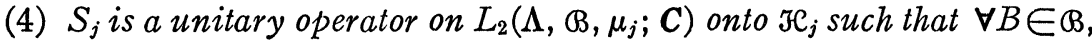
Rstr. $\mathfrak{F c}_{j} E(B)=S_{j} \circ M_{\chi_{B}} \circ S_{j}^{-1}$ where $M_{\chi_{B}}$ is the operation of multiplication by the indicator-function $\chi_{B}$.

Indeed, on applying Thm.14.3 with $\Lambda=\hat{X}$ and $B=\hat{B}$ to the spectral measure $E(\cdot)$ obtained in 14.1 , we can get Thm. 14.2 by using the operational calculus. Similarly, from Thm. 14.3 with $\Lambda=R$ and $B=$ the Borel family over $R$, we can get the representation, corresponding to 14.2 , of a selfadjoint operator $H$ from the Spectral Theorem for $H$.

B. Our aim is to prove not Theorem 14.3 but rather a more explicit and concise version of this theorem. The procedure for accomplishing this usually adopted in operator-algebras, is to solder the measurespaces $\left(\Lambda, \mu_{j}\right)$, and so get a single nonnegative measure $\mu$ over a "large space" $\mathfrak{X}$, cf. Segal \& Kunze [45, p. 89-]. But Cramer's fundamental paper [8] on matrix-valued distributions, and the ensuing development of the theory of multivariate random processes and fields, cf. [29], [51], suggest that it would be better in most respects to view the $\mu_{j}$ as components of an operator-valued measure $M$ over $\Lambda$ itself..$^{39}$ Accordingly, our goal will be to formulate Thm. 14.3 in terms of integrals involving a c.a.q.i. measure for which the nonnegative hermitian measure is built from the $\mu_{j}$ by following Procedure 12.1. The key to this lies in the following very simple lemma, which says roughly that the restriction of a spectral measure is c.a.q.i. with respect to a certain nonnegative hermitian measure built from it:

14.4. Lemma. Let

(i) $E(\cdot)$ be a spectral measure for $\mathcal{H C}$ on a $\sigma$-algebra $B$ over a set $\Lambda$,

(ii) $W$ be any subspace of $\mathcal{H}$, and $P_{W}$ be the orthogonal projection on Je onto $W$,

(iii) $E_{0}(\cdot)=\operatorname{Rstr}_{W} E(\cdot), \quad M_{0}(\cdot)=P_{W} \circ\left\{E_{0}(\cdot)\right\}$.

(a) $M_{0}(\cdot)$ is a strongly c.a., W-to- $W$, nonnegative hermitian, contraction-valued measure on $B \ni M_{0}(\Lambda)=\mathrm{Rstr}_{\mathrm{W}} I$;

${ }^{39}$ Indeed, the factorization theorems proved in prediction theory for the (operatorvalued) Radon-Nikodým derivatives of such measures, cf. e.g. [34], have stimulated new work in operator algebras, cf. e.g. Arveson $[1, \S 4]$. 
(b) $E_{0}(\cdot)$ is a W-to-JC, c.a.q.i. measure over $\left(\Lambda, @, M_{0}\right)$.

Proof. (a) Let $B \in ß$. Then by (iii), $M_{0}(B)$ is a continuous linear operator on $W$ to $W$. It is also easy to check from (iii) that $\forall w, w^{\prime} \in W$

$$
\left(M_{0}(B)(w), w^{\prime}\right)_{W}=\left(E(B)(w), E(B)\left(w^{\prime}\right)\right)_{W}=\left(w, M_{0}(B)\left(w^{\prime}\right)\right)_{W} .
$$

From this it follows at once that $M_{0}(B)$ is hermitian and nonnegative definite. It is a contraction, since

$$
\forall w \in W, \quad\left|M_{0}(B)(w)\right|_{W}=\left|P_{W} E(B)(w)\right|_{W} \leqq|w|_{w} .
$$

Also, $\forall w \in W, \quad M_{0}(\Lambda) w=P_{W} E(\Lambda) w=w$, and so $M_{0}(\Lambda)=\operatorname{Rstr} . W I$. Finally, $M_{0}(\cdot)$ is s.c.a., since $E(\cdot)$ is s.c.a.

(b) Let $A, B \in B$. Clearly $E_{0}(A)$ is a continuous linear operator on $W$ to $\mathfrak{H}$, and $\forall w, w \in W$,

$$
\begin{aligned}
\left(E_{0}(A)(w), E_{0}(B)\left(w^{\prime}\right)\right)_{\Im C} & =\left(E(A)(w), E(B)\left(w^{\prime}\right)\right)_{\Im C} \\
& =\left(E(A \cap B)(w), w^{\prime}\right)_{\Im C} \\
& =\left(E(A \cap B)(w), P_{W}\left(w^{\prime}\right)\right)_{\Im C} \\
& =\left(M_{0}(A \cap B)(w), w^{\prime}\right)_{W} .
\end{aligned}
$$

Hence, cf. (8.3), we have (b).

To discuss the basicness of the measure $E_{0}(\cdot)$ of 14.4 we must recall the following concepts from spectral multiplicity theory:

14.5. Definition. Let $E$ be a spectral measure for a (complex) Hilbert space $\mathcal{H}$, defined on a $\sigma$-algebra $B$ over a set $\Lambda$. Then

(a) a subset $G$ of $\mathcal{H C}$ is called a generating set of $E(\cdot)$, iff

$$
\Im\{E(B)(G): B \in B\}=\mathfrak{H} \text {; }
$$

(b) the minimum of the cardinality of all such subsets $G$ is called the (total) multiplicity of $E(\cdot)$.

It follows at once from 14.4 (b) that

the c.a.q.i. measure $E_{0}(\cdot)$ of 14.4 is $W$-to-JC basic, iff $W=\mathfrak{S}(G)$, for some generating set $G$ of $E(\cdot)$.

Spectral multiplicity considerations thus enter into our theory in regard to the basicness of the c.a.q.i. measure $E_{0}(\cdot)$. But they also confront us in another significant way. To be able to use $E_{0}(\cdot)$ successfully for integration $(\$ 10)$, we must know beforehand that $L_{2}\left(\Lambda, \Theta, M_{0} ; W\right)$ is definable as a Hilbert space (\$9). Thus arises the question: 
QUESTION. For what generating sets $G$ of $E(\cdot)$ will the measure $M_{0}(\cdot)$ of 14.4 be adequate according to Criterion 9.9 ?

If, for instance, we choose the largest $G$, viz. $G=\mathcal{H C}$, in which case obviously $E_{0}=E=M_{0}$, we get $L_{2}(\Lambda, B, E ; \mathfrak{H C})$, and it is not clear what this symbol means. We shall now show that a careful minimal choice of $G$ yields an adequate $M_{0}(\cdot)$. This will be done by appealing to the Hellinger-Hahn Theorem. Indeed, from our present standpoint the significance of this theorem lies in its ability to perform this measuretheoretic role. The following version based on Dunford and Schwartz [11, II, p. 914-] is needed:

14.7. Hellinger-Hahn Theorem. Let

(i) $E$ be a spectral measure for $\mathcal{H C}$ on $B$,

(ii) $q={ }_{d}$ the multiplicity of $E \leqq \boldsymbol{N}_{0},(c f .14 .4)$,

(iii) $\forall x \in \mathcal{H}, \delta_{x}={ }_{d} \subseteq\{E(B)(x): B \in B\}, \quad \mu_{x}(\cdot)={ }_{d}|E(\cdot)(x)|^{2}$,

(iv) $J$ be the initial segment of cardinality $q$ of the naturally ordered set of positive integers.

Then $\exists$ a sequence $\left(\beta_{j}\right)_{j \in J}$ of vectors in Fe such that

(a) $\quad \mathcal{C}=\sum_{j \in J} \delta_{\beta_{j}}, \quad \delta_{\beta_{j}} \perp \delta_{\beta_{k}}, \quad\left|\beta_{j}\right|=1, j, k \in J, \quad j \neq k$;

$$
\forall j \in J, \quad \mu_{\beta_{j}}(\cdot)=\mu_{\beta_{1}}\left(\Lambda_{j} \cap \cdot\right) / a_{j},
$$

where $\Lambda_{j} \in ß \quad \& \Lambda_{k} \subseteq \Lambda_{j} \subset \Lambda_{1}=\Lambda, \forall j, k \in J, k \geqq j$, and $a_{j}={ }_{d} \mu_{\beta_{1}}\left(\Lambda_{j}\right)>0$.

14.8. Definition. Let (i)-(iv) be as in 14.7. Then we shall call a sequence $\left(\beta_{j}\right)_{j \in J}$ satisfying (a) and (b) a Hellinger-Hahn (H.H.) sequence for $E . \mu_{\beta_{1}}$ will be called its dominant measure and $\left(\Lambda_{j}, j \in J\right)$ its carrier sequence.

It is known that the range $G$ of a H.H. sequence $\left(\beta_{j}\right)_{j \in J}$ for $E(\cdot)$ is a minimal generating set for $E(\cdot)$, and the sequence itself is an o.n. basis for the separable subspace $W={ }_{d} \subseteq(G)$. Since $\left|\beta_{j}\right|=1$, all $\mu_{\beta_{j}}(\cdot)$ are probability measures on $B$. Furthermore, cf. [11, II, p. 916, Thm.10] for two H.H. sequences for the same $E(\cdot)$ the dominant measures are mutually absolutely continuous, and the symmetric differences of corresponding carriers have zero dominant measure.

The following theorem shows that for the subspace $W$ spanned by a H.H. sequence for $E(\cdot)$, the measure $M_{0}(\cdot)$ of 14.4 is adequate, and the c.a.q.i. integral is a convergent sum of c.a.o.s. integrals:

14.9. Theorem. Let (i)-(iv) be as in the Hellinger-Hahn Thm.14.7.

(v) $\left(\beta_{j}\right)_{j \in J}$ be a $H . H$. sequence for $E(\cdot)$.

(vi) $W={ }_{d} \subseteq\left\{\beta_{j}: j \in J\right\}$, 
(vii) $E_{0}(\cdot)=\operatorname{Rstr}_{W} E(\cdot), M_{0}(\cdot)=P_{W} \circ\left\{E_{0}(\cdot)\right\}$ (cf. 14.4). Then

(a) $E_{0}$ is a W-to-Je basic, c.a.q.i. meas. over $\left(\Lambda, \Theta, M_{0}\right)$;

(b) $\forall B \in B, M_{0}(B)=\sum_{j \in J} \mu_{\beta_{j}}(B) P_{\beta_{j}}$, where $P_{\beta_{j}}$ is the orthogonal projection on $W$ onto the 1-dimensional subspace $\mathfrak{S}\left(\beta_{j}\right)$, and where strong convergence is understood for infinite $J$;

(c) the measure $M_{0}$ is adequate; hence $\mathfrak{L}_{2, W}={ }_{d} L_{2}\left(\Lambda, \Theta, M_{0} ; W\right)$ is a Hilbert space, and the linear manifold $s(\Lambda, B ; W)$ of $\Theta$-simple functions on $\Lambda$ to $W$ is e.d. in it;

(d) $\forall \phi \in \mathfrak{L}_{2}, W \& \forall B \in \Theta$,

$$
\int_{B} E_{0}(d \lambda) \phi(\lambda)=\sum_{j \in J} \int_{B}\left(\phi(\lambda), \beta_{j}\right)_{W} E_{0}(d \lambda) \beta_{j} .
$$

Proof. (a) By $14.4(\mathrm{~b}), E_{0}$ is a $W$-to-FC, c.a.q.i. measure over $\left(\Lambda, \bigotimes, M_{0}\right)$. Now cf. 14.8 et seq., by Thm. 14.7(a) the range $G$ of $\left(\beta_{j}\right)_{j \in J}$ is a generating set for $E(\cdot)$, and by (vi), $W=\Im(G)$. Hence by (14.6), $E_{0}$ is $W$-to-JC basic.

(b) Let $B \in ß$. Then

$$
\forall j \in J, \quad M_{0}(B)\left(\beta_{j}\right)=P_{W}\left\{E(B) \beta_{j}\right\} \in W .
$$

Since, cf. 14.8 et seq., $\left(\beta_{j}\right)_{j \in J}$ is an o.n. basis for $W$, it follows that

$$
\begin{aligned}
\forall j \in J, \quad M_{0}(B)\left(\beta_{j}\right) & =\sum_{k \in J}\left(E(B) \beta_{j}, \beta_{k}\right) \beta_{k}, \\
& =\left(E(B) \beta_{j}, \beta_{j}\right) \beta_{j}, \quad \text { since } \delta_{\beta_{j}} \perp \delta_{\beta_{k}} \\
& =\mu_{\beta_{j}}(B) \beta_{j} .
\end{aligned}
$$

Hence $\forall w \in W$, it follows on using the continuity of $M_{0}(B)$ that

$$
\begin{aligned}
M_{0}(B)(w) & =M_{0}(B)\left\{\sum_{j \in J}\left(w, \beta_{j}\right) \beta_{j}\right\}=\sum_{j \in J}\left(w, \beta_{j}\right) \mu_{\beta_{j}}(B) \beta_{j} \\
& =\sum_{j \in J} \mu_{\beta_{j}}(B) P_{\beta_{j}}(w) .
\end{aligned}
$$

This yields (b).

(c) follows from $\$ 9$, since $M_{0}$ satisfies $(9.16)$ and $(9.20)$ by dint of (b) and 14.7 (b).

(d) clearly follows from Thm.10.32(d) on setting $T(\cdot)=E_{0}(\cdot)$.

Theorem 14.9 completes steps (i) and (ii) of Procedure 12.1. Our Isomorphism Theorem 10.8 now tells us that

$$
\begin{aligned}
& \text { the correspondence } \Sigma: \phi \rightarrow \int_{\Delta} E_{0}(d \lambda) \phi(\lambda) \\
& \text { is a unitary operator on } \mathfrak{L}_{2, W} \text { onto } \mathcal{H C} \text {. }
\end{aligned}
$$


We next assert that this $\Sigma$ carries the operation of multiplication by $\chi_{B}$ on $\mathfrak{L}_{2, W}$ into the operation $E(B)$ on $\mathcal{F C}$ :

14.11. Lemma. Let $\Sigma$ be defined as in 14.10. Then

$$
\forall B \in \mathbb{B}, \quad E(B)=\Sigma \circ M_{x_{B}} \circ \Sigma^{-1} .
$$

Proof. Let $B \in B$ and $\phi \in \mathfrak{L}_{2, w}$. In view of (14.10) it will suffice to show that $E(B)\{\Sigma(\phi)\}=\Sigma\left\{\chi_{B}(\cdot) \phi(\cdot)\right\}$, i.e.

$$
E(B)\left\{\int_{\Delta} E_{0}(d \lambda) \phi(\lambda)\right\}=\int_{\Delta} E_{0}(d \lambda)\left\{\chi_{B}(\lambda) \phi(\lambda)\right\} .
$$

Case (i). Let $\phi=\sum_{1}^{r} w_{k} \chi_{A_{k}}, w_{k} \in W \& A_{k} \in ß$. Then from Definition 10.4 , the linearity of $E(B)$ and its commutativity with $E\left(A_{k}\right)$, we get

$$
\operatorname{LHS}(\mathrm{I})=E(B)\left\{\sum_{1}^{r} E_{0}\left(A_{k}\right)\left(w_{k}\right)\right\}=\sum_{1}^{r} E\left(B \cap A_{k}\right)\left(w_{k}\right) .
$$

On the other hand, from the linearity of our c.a.q.i. integrals, cf. $10.3(\mathrm{c})$,

$$
\begin{aligned}
\operatorname{RHS}(\mathrm{I}) & =\int_{\Lambda} E_{0}(d \lambda)\left\{\sum_{1}^{r}{\chi_{B \cap A_{k}}}(\lambda) w_{k}\right\} \\
& =\sum_{1}^{r} \int_{\Lambda} E_{0}(d \lambda)\left\{\chi_{B \cap A_{k}}(\lambda) w_{k}\right\} \\
& =\sum_{1}^{r} \int_{\Delta} \chi_{B \cap A_{k}}(\lambda) E_{0}(d \lambda)\left(w_{k}\right), \quad \text { by } 10.10 \\
& =\sum_{1}^{r} E\left(B \cap A_{k}\right)\left(w_{k}\right),
\end{aligned}
$$

where the last step follows from the operational calculus. Thus (I) holds in Case (i).

Case (ii). Let $\phi=\lim _{n \rightarrow \infty} \phi_{n}$ in $\mathfrak{L}_{2, W}$, where $\phi_{n}$ is as in Case (i). Then, cf. Definition 10.6,

$$
\begin{aligned}
\operatorname{LHS}(\mathrm{I}) & =E(B)\left\{\lim _{n \rightarrow \infty} \int_{\Delta} E_{0}(d \lambda) \phi_{n}(\lambda)\right\} \\
& =\lim _{n \rightarrow \infty} E(B)\left\{\int_{\Delta} E_{0}(d \lambda) \phi_{n}(\lambda)\right\} \\
& =\lim _{n \rightarrow \infty} \int_{\Delta} E_{0}(d \lambda)\left\{\chi_{B}(\lambda) \phi_{n}(\lambda)\right\}, \quad \text { by Case (i), } \\
& =\operatorname{RHS}(\mathrm{I}),
\end{aligned}
$$


where the last step follows from 10.3(e), since $\chi_{B}(\cdot) \phi(\cdot)=$ $\lim _{n \rightarrow \infty} \chi_{B}(\cdot) \phi_{n}(\cdot)$ in $\mathfrak{L}_{2, w}$. Thus (I) holds in Case (ii).

To sum up, we have proved the following theorem which is an explicit and concise substitute of the spectral representation 14.3:

14.12. Theorem (SPECTRAL Representation II). Let

(i) $E(\cdot)$ be a spectral measure for $\mathcal{H C}$ on a $\sigma$-algebra $B$ over a set $\Lambda$, such that $q={ }_{d}$ multiplicity of $E(\cdot) \leqq \boldsymbol{N}_{0}$,

(ii) $\left(\beta_{j}\right)_{j \in J}$ be a Hellinger-Hahn sequence for $E(\cdot)$, and

$$
W=\underset{d}{=} \subseteq\left\{\beta_{j}: j \in J\right\}, \quad \text { cf. 14.8, }
$$

(iii) $E_{0}(\cdot)={ }_{d} \operatorname{Rstr} . W E(\cdot), M_{0}(\cdot)=P_{W} \circ\left\{E_{0}(\cdot)\right\}$.

Then the correspondence $\Sigma: \phi \rightarrow \int_{\Lambda} E_{0}(d \lambda) \phi(\lambda)$ is a unitary operator on $L_{2}\left(\Lambda, B, M_{0} ; W\right)$ onto 3 such that

$$
\forall B \in B, \quad E(B)=\Sigma \circ M_{\chi_{B}} \circ \Sigma^{-1} .
$$

14.13. Remarks. 1 . The ingredients $W, E_{0}, M_{0}$ in the last theorem are constructed explicitly from the given spectral measure $E(\cdot)$. The subspace $W$ is not unique, and so neither are the measures $E_{0}$ and $M_{0}$. But $W, E_{0}, M_{0}$ are determined up to unitary equivalence, and this is the most that our data will allow. Thus Thm. 14.12 is as explicit as possible, apart from being more concise than 14.3.

2. Thm. 14.12 shows incidentally that $F(\cdot)$, where

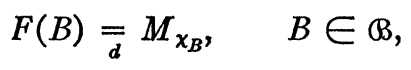

is a spectral measure for the Hilbert space $L_{2}\left(\Lambda, \Theta, M_{0} ; W\right)$, when $W$ and $M_{0}$ are defined as in the last theorem.

3. The spectral measure $E(\cdot)$ itself is the spatial integral spectral measure $Q(\cdot)$ for the c.a.q.i. measure $E_{0}(\cdot)$. This is clear on combining 14.11 and $10.25(\mathrm{c})$, or alternatively on combining the equation (I) in the proof of 14.11 and $10.25(\mathrm{~b})$.

C. We shall now turn to the explication of the representation for $\left(U_{x}, x \in X\right)$ given in 14.2 and of the corresponding representation for a selfadjoint operator:

14.14. STONE's TheOREM (Form III). Let

(i) $\left(U_{x}, x \in X\right)$ and $E(\cdot)$ be as in Theorem 14.1, and

$$
q={ }_{d} \text { multiplicity of } E(\cdot) \leqq \boldsymbol{\aleph}_{0},
$$

(ii) $\left(\beta_{j}\right)_{j \in J}$ be a Hellinger-Hahn sequence for $E$ and 


$$
\left.W=\Im \beta_{j}: j \in J\right\}, \quad c f .14 .8
$$

(iii) $E_{0}(\cdot)={ }_{d}$ Rstr. $E(\cdot), \quad M_{0}(\cdot)={ }_{d} P_{W} \circ\left\{E_{0}(\cdot)\right\}$.

Then the correspondence $\Sigma: \phi \rightarrow \int_{\hat{X}} E_{0}(d \alpha) \phi(\alpha)$ is a unitary operator on $L_{2}\left(\hat{X}, \hat{A}, M_{0} ; W\right)$ onto $\mathfrak{H C}$ such that

$$
\forall x \in \mathcal{H}, \quad U_{x}=\Sigma \circ M_{[x, \cdot]} \circ \Sigma^{-1} .
$$

Proof. By Thm. 14.12, the correspondence $\Sigma$ just defined is a unitary operator on $L_{2}\left(\hat{X}, \hat{\mathscr{B}}, M_{0} ; W\right)$ onto $\mathfrak{H C}$ such that

$$
\forall B \in \hat{B}, \quad E(B)=\Sigma \circ M_{\chi_{B}} \circ \Sigma^{-1},
$$

Now let $x \in X$. Then by (1) and the operational calculus, cf. Remark $14.13(2)$,

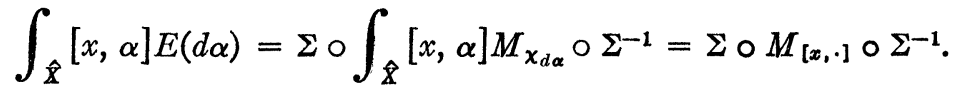

Since by Thm. 14.1, $\operatorname{LHS}(2)=U_{x}$, the proof is over.

In very much the same way we deduce from Thm. 14.12 the following analogous result for selfadjoint operators:

14.15. TheOREM (SPECTRAL REPRESENTATION FOR S.A. OPERATOR). Let

(i) $H$ be any selfadjoint, linear operator from $\mathfrak{H C}$ to $\mathfrak{H C}$, and $E(\cdot)$ be its associated spectral measure on the $\sigma$-algebra $\mathrm{Bl}(R)$ of Borel sets,

(ii) $q={ }_{d}$ multiplicity of $E(\cdot) \leqq \aleph_{0}$,

(iii) $\left(\beta_{j}, j \in J\right), W, E_{0}, M_{0}$ be defined in terms of $E(\cdot)$ as in the last theorem.

Then the correspondence $\Sigma: \phi \rightarrow \int_{R} E_{0}(d \lambda) \phi(\lambda)$ is a unitary operator on $L_{\mathbf{2}}\left(R, \mathrm{Bl}(R), M_{0} ; W\right)$ onto HC such that

$$
H=\Sigma \circ M_{I(\cdot)} \circ \Sigma^{-1}, \quad \text { where } \quad I(\lambda)=\lambda, \quad \lambda \in R .
$$

In conclusion we remark that Thm. 14.14 is as explicit as possible. It is more concise than 14.2 , and reveals a closer connection to 14.1 than does 14.2 . Indeed, the very definitions of $W, E_{0}, M_{0}$ involve the spectral measure $E$ of Thm. 14.1, which as mentioned in part A may be thought of as a barycentric operator-valued measure stemming from the convexity underlying the situation. Similar remarks apply to 14.15 .

\section{Linear stationary causal systems and Cooper's theorem}

Many linear problems involve a Hilbert space $\mathcal{H}$ and a strongly 
continuous group $\left(U_{t}, t \in R\right)$ of unitary operators on $\mathcal{H C}$ onto $\mathcal{H C}$. If for $x \in \mathcal{H C}$ we interpret $U_{t}(x)$ as being the state $x_{t}$ at instant $t$ of a system whose initial state is $x$, then $\left|x_{t}\right|^{2}$ and $\left(x_{t}, y_{t}\right)$ are time-invariant. This justifies calling such a system stationary. Stone's Theorem 14.1 for $X=R$ provides a useful tool for the analysis of linear, stationary systems.

In an important subclass of linear, stationary systems there exist subspaces $\mathcal{H}_{0}$ of $\mathscr{H}$ which are invariant under the semigroup $\left(U_{t}\right.$, $t \leqq 0)$, i.e.

$$
U_{t}\left(\mathcal{H}_{0}\right) \subseteq \mathcal{H}_{0}, \quad \forall t \leqq 0 .
$$

As this signifies a discrimination of past from future, we may call such systems causal.40

15.2. ExAmples. The closely related theories of (i) the Hardy class $H_{2}$, (ii) prediction and (iii) scattering exemplify linear, stationary, causal systems. In (i) $\mathcal{H}=L_{2}(R), U_{t}$ is multiplication by $e_{-t}$ where $e_{t}(s)=e^{i t s}$, and $\mathcal{H}_{0}={ }_{d} L_{2}^{0+}(R)$, i.e. the set of functions $f \in \mathcal{H C}$ which are boundary values of functions $f_{+}$in the Hardy class $H_{2}$ on the upper bank of $R$. In (ii) $\mathfrak{H C}={ }_{d} \mathfrak{S}\left(x_{t}, t \in R\right.$ ) is the Hilbert space generated by a stationary stochastic process, $U_{t}$ is such that $U_{t}\left(x_{s}\right)=x_{t+8}$, and $\mathcal{H C}_{0}={ }_{d} \subseteq\left(x_{t}, t \leqq 0\right)$, the so-called "present and past space" of $t=0$. In (iii) $\mathcal{H}$ is the space of Cauchy-data of the wave equation with obstacle in $R^{q}, q \geqq 1$, completed in the energy norm, $U_{t}$ carries the initial data $\left(f_{0}, g_{0}\right)$ into the data $\left(f_{t}, g_{t}\right)$ at instant $t$, and $\mathcal{H}_{0}$ is the so-called "incoming subspace," i.e. the set of all initial data $\left(f_{0}, g_{0}\right)$ such that for all $t \leqq 0, f_{t}$ vanishes in a (fixed) neighborhood of the obstacle. It is easy to verify (15.1) in each case. let

Now suppose that we have a linear, stationary, causal system, and

$$
\forall t \geqq 0, \quad S_{t}=\operatorname{Rstr}_{x_{0}} U_{t}^{*}
$$

Then by (15.1), $\left(S_{t}, t \geqq 0\right)$ is a strongly continuous semigroup of iso metries on $\mathfrak{H}_{0}$ into $\mathfrak{H}_{0}$. The following theorem of Cooper [7], which gives the structure of such semigroups, therefore plays an important role in the analysis of linear, stationary, causal systems:

15.4. CoOper's TheOREM. Let $\left(S_{t}, t \geqq 0\right)$ be a strongly continuous semigroup of isometries on a (complex) Hilbert space HC into HC. Then there exists a subspace $\mathfrak{H}_{\infty}$ and indexed families $\left(\mathfrak{T}_{j}, j \in J\right),\left(Z_{j}, j \in J\right)$

${ }^{40}$ Causality is used here in the sense of temporal anisotropy, and not of course in the narrower sense of determinism. 
of subspaces and maps, respectively, such that

(1) $\mathfrak{H C}=\mathfrak{H}_{\infty}+\sum_{j \in J} \mathfrak{M}_{j}, \quad \mathfrak{C}_{\infty} \perp \mathfrak{M}_{j} \perp \mathfrak{M C}_{k}, j, k \in J, \quad j \neq k$,

(2) $\forall t \geqq 0, S_{t}\left(\mathfrak{H}_{\infty}\right) \subseteq \mathcal{H}_{\infty} \quad \& \quad S_{t}\left(\mathscr{T H}_{j}\right) \subseteq \mathbb{M}_{j}$,

(3) $\forall t \geqq 0$, Rstr. $\mathcal{H}_{\infty} S_{t}$ is unitary on $\mathfrak{H C}_{\infty}$ onto $\mathfrak{H C}_{\infty}$,

(4) $\forall j \in J, Z_{j}$ is a unitary operator on $L_{2}[0, \infty)$ onto $\mathfrak{T l}_{j}$ such that

$$
\forall t \geqq 0, S_{t}=Z_{j} \circ \sigma_{t} \circ \bar{Z}_{j}^{1}, \text { where }\left(\sigma_{c} f\right)(t)= \begin{cases}f(t-c), & t \geqq c \\ 0, & 0 \leqq t<c .\end{cases}
$$

To get the $\Re_{j}$ Cooper first obtains vectors $\beta_{j}$ in $\mathcal{H}$ by a selection procedure reminiscent of the Hellinger-Hahn Theorem. ${ }^{41}$ For the analysis of linear, stationary, causal systems what is needed is a version of his theorem free from any dependence on a nonunique selection of vectors. Our aim is to get such a version by following Procedure 12.1. To carry out step (i) of 12.1 we shall appeal to the corresponding result for the semigroup $\left(V^{n}, n \geqq 0\right)$ where $V$ is an isometry on $\mathfrak{H C}$ into $\mathfrak{H}$, and then make a transition from the discrete to the continuous, cf. 1.3. In the discrete case the result we are seeking is in essence a mere reformulation of the following fundamental result, cf. Halmos [14, Lma. 1]:

15.5. TheOREM (DISCRETE WOLD DECOMPOSITION). Let $V$ be an isometry on a (complex) Hilbert space $\mathfrak{H C}$ into $\mathcal{H C}$. Then $\exists$ a unique subspace $W$ such that

and

$$
\mathfrak{H C}=\bigcap_{0}^{\infty} V^{n}(\mathfrak{H C})+\sum_{n=0}^{\infty} V^{n}(W)
$$

$$
\bigcap_{0}^{\infty} V^{n}(\mathcal{H C}) \perp V^{j}(W) \perp V^{k}(W), \quad j>k \geqq 0 \text {. }
$$

This $W$ is $\{V(\mathcal{F C})\} \perp$.

It is easy to see that the restriction of $V$ to $\mathfrak{H C}_{\infty}={ }_{d} \bigcap_{0}^{\infty} V^{n}(\mathfrak{H C})$ is unitary. Also, on letting $l_{2}\left(\mathscr{N}_{0+} ; W\right)$ be the Hilbert space of squaresummable functions on the set $\mathscr{N}_{0+}$ of nonnegative integers to the space $W$, it easily follows from 15.5 that

$$
\Sigma: \phi(\cdot) \rightarrow \sum_{n=0}^{\infty} V^{n}\{\phi(n)\}
$$

${ }^{41}$ As Kallianpar and Mandrekar [19] have pointed out, Cooper's method in essence amounts to constructing from each $\beta_{j}$, a c.a.o.s. measure $\xi_{j}$, and then taking $\mathfrak{I T}_{j}$ to be the subspace of $\xi_{j}$, cf. 2.2(a). 
is a unitary operator on $l_{2}\left(\Re_{0+} ; W\right)$ onto $\mathfrak{H} \cap \mathcal{H}_{\infty}^{+}$, and $\forall n \geqq 1$, $V^{n}=\Sigma \circ \sigma_{-n} \circ \Sigma^{-1}$, where $\sigma_{n}$ is defined as in 15.4(4). Thus, in the discrete case the Wold decomposition immediately yields an explicit version of Cooper's Theorem.

The last fact suggests that in the continuous parameter case as well, an explicit version of Cooper's Theorem may be derivable from a Wold decomposition established beforehand. Indeed, the equation in 15.5 strongly suggests for the semigroup $\left(S_{t}, t \geqq 0\right)$ a Wold decomposition of the form

$$
\mathcal{H}=\bigcap_{t \geq 0} S_{t}(\mathcal{H C})+\int_{0}^{\infty} T_{0}(d t)(W)
$$

where $W$ is a unique subspace of $\mathcal{H}$ and $T_{0}(\cdot)$ a $W$-to- $\mathcal{H}$, c.a.q.i. measure over $\left([0, \infty), \Theta_{\mu}, \mu\right)$, where $\mu$ is Lebesgue measure, and $\cap_{\mu}$ is the $\delta$-ring of Borel subsets of $[0, \infty)$ of finite Lebesgue measure. In the announcement [28] we sketched a proof of (1). We shall now present this in a somewhat improved and systematized form. To avoid repetition let it be understood in what follows that:

(i) $\quad\left(S_{t}, t \geqq 0\right)$ is strongly continuous semigroup of isometries on a (complex) Hilbert space $\mathfrak{H C}$ into $\mathfrak{H}$,

(ii) $\quad \mathfrak{H}_{\infty}=\bigcap_{t_{\mathbb{2} 0}} S_{t}(\mathfrak{H C}) \quad \& \quad \mathfrak{H}_{0}=\mathfrak{H C}_{\infty}^{\perp}$,

(iii) $\quad V={ }_{d} I-2 \int_{0}^{\infty} e^{-t} S_{t} d t \quad \& \quad W=\{V(\mathcal{H C})\}^{\perp, 42}$

(15.6) (iv) $\odot$ is the prering of intervals $(a, b]$, where $0 \leqq a<b<\infty$,

(v) $\forall(a, b] \in \mathcal{P}, T(a, b]={ }_{d}(1 / \sqrt{ } 2)\left\{S_{b}-S_{a}-\int_{a}^{b} S_{t} d t\right\}$,

(vi) $\quad T_{0}(\cdot)={ }_{d} \quad \operatorname{Rstr}_{W} T(\cdot) \quad \& \quad I_{W}={ }_{d}$ Rstr.WI,

(vii) $\quad \forall t \geqq 0, \quad R_{t}={ }_{d} \quad S_{t}-I-\int_{0}^{t}(t-s) S_{s} d s$.

Our goal is to show that $T_{0}(\cdot)$ is a $W$-to-JC, c.a.q.i. measure over $\left([0, \infty), \wp_{\mu}, \mu(\cdot) I_{W}\right)$, where $\mu$ is Lebesgue measure, and then use Thm. 10.8 to get both (1) and the explicit version of 15.4 , thereby carrying out Procedure 12.1. But the unrestricted set-function $T(\cdot)$ must first engage our attention.

In the first place, the semigroup (15.6)(i) yields a continuous function $S_{(\cdot)}$ on $[0, \infty)$ to the vector space CL(FC, $\left.\mathfrak{F}\right)$ taken with the strong operator topology. Hence for $0 \leqq a<b<\infty, \int_{a}^{b} S_{t} d t$ exists as a strong Riemann integral, which obeys many of the usual laws (cf. $[18$, p. $62-$, Def. 3.3.1 \& Thm. 3.3.4]). Hence $T(a, b]$ is well defined by $(15.6)(v)$, and it follows easily that

${ }^{42}$ Thus $V$ is the Cayley transform of $H$, where $i H$ is the infinitesimal generator of $\left(S_{t}, t \geqq 0\right)$, and $W$ is the so-called deficiency subspace of the semigroup. As Cooper showed in [6], $H$ is a maximal symmetric operator. 
(a) $T(\cdot)$ is a finitely additive measure on $\odot$ to $\mathrm{CL}(\mathcal{H C}, \mathfrak{H})$,

(b) $\quad \forall t \geqq 0 \& \forall A \in \mathcal{P}, \quad S_{t} \circ\{T(A)\}=T(A+t)$.

Furthermore, the equation in $(15.6)(\mathrm{v})$ for $T(\cdot)$ in terms of $S_{t}$ can be inverted to yield an equation for $S_{t}$ in terms of $T(\cdot)$ :

15.8. LEMMA (INVERSION FORMULA).

$$
\forall a \geqq 0, \quad S_{a}=-\sqrt{ } 2 \int_{0}^{\infty} e^{-t} T(a, a+t] d t .
$$

Proof. In view of (15.7)(b) we shall prove the result for $a=0$, viz.

$$
-\sqrt{ } 2 \int_{0}^{\infty} e^{-t} T(0, t] d t=I,
$$

and then apply $S_{a}$ to both sides. By $(15.6)(\mathrm{v}) \&(\mathrm{i}), T(0, \cdot]$ is a continuous function on $[0, \infty)$ to the space $\mathrm{CL}(\mathcal{H C}, \mathfrak{H C})$ taken with the strong operator topology. Hence, the function $e^{-(\cdot)} T(0, \cdot]$ is likewise continuous on $[0, \infty)$ and therefore Riemann integrable on any interval in $\odot$. But from (15.6)(v)

$$
\begin{aligned}
\forall t \geqq 0, \quad|T(0, t]|_{B} & \leqq\left|S_{t}\right|_{B}+|I|_{B}+\int_{0}^{t}\left|S_{B}\right|_{B} d s \\
& \leqq 2+t .
\end{aligned}
$$

It follows that $\int_{0}^{\infty} e^{-t} T(0, t] d t$ exists as an improper $R$-integral. Hence (A) is meaningful.

Next, we observe that

$$
\begin{aligned}
\operatorname{LHS}(\mathrm{A}) & =\int_{0}^{\infty} e^{-t}\left\{-S_{t}+I+\int_{0}^{t} S_{s} d s\right\} d t \\
& =-\int_{0}^{\infty} e^{-t} S_{t} d t+I+\int_{0}^{\infty}\left\{\int_{0}^{t} e^{-t} S_{s} d s\right\} d t .
\end{aligned}
$$

By Dirichlet's formula the last term on the RHS is

$$
\int_{0}^{\infty}\left\{\int_{\varepsilon}^{\infty} e^{-t} S_{s} d t\right\} d s=\int_{0}^{\infty} S_{s} e^{-s} d s,
$$

and so cancels the first term, to yield $I$ on the RHS, as required by (A).

Finally, from (A) and (15.7)(b) 


$$
\begin{aligned}
S_{a} & =-\sqrt{ } 2 \int_{0}^{\infty} e^{-t} S_{a} T(0, t] d t \\
& =-\sqrt{ } 2 \int_{0}^{\infty} e^{-t} T(a, a+t] d t .
\end{aligned}
$$

Let us note that

$$
\begin{aligned}
& \forall X \subseteq \mathcal{F C} \& \forall a \geqq 0 \\
& \quad \subseteq\left\{\mathrm{S}_{t}(X): t \geqq a\right\}=\subseteq\{T(c, d](X):(c, d] \subset[a, \infty)\} .
\end{aligned}
$$

This is clear since on the one hand by (15.6) (v), we have $\forall(c, d]$ $\subset[a, \infty)$ and $\forall x \in X$,

$$
\begin{gathered}
T(c, d](x)=(1 / \sqrt{ } 2) \\
\left\{S_{d}(x)-S_{c}(x)-\int_{c}^{d} S_{t}(x) d t\right\} \\
\in \subseteq\left\{S_{t}(X): t \geqq a\right\},
\end{gathered}
$$

and on the other hand by the formula in 15.8 , we have $\forall t \geqq a$ and $\forall x \in X$,

$$
\begin{array}{rl}
S_{t}(x)=-\sqrt{ } 2 \int_{0}^{\infty} e^{-s} & T(t, t+s](x) d s \\
& \in \subseteq\{T(c, d](X):(c, d] \subset[a, \infty)\} .
\end{array}
$$

The next result we prove concerning $T(\cdot)$ is chosen with an eye to establishing the quasi-isometry of $T_{0}(\cdot)$ by appeal to Lma. 8.12:

15.9. LEMMA.

(a) $\quad 2 T(0, a]^{*} \circ T(0, b]=R_{b-a}-R_{a}^{*}-R_{b}, \quad 0 \leqq a<b$,

(b) $\quad 2 T(0, b]^{*} \circ T(0, a]=R_{b}{ }^{*}-a-R_{a}-R_{b}{ }^{*}, \quad 0 \leqq a<b$.

Proof. The proof consists of a long but routine calculation. The following are the main steps. Let $0 \leqq a<b$. Then using the relations

$$
S_{a}^{*} S_{b}=S_{b-a} \& S_{b}^{*} S_{a}=S_{b-a}^{*},
$$

we find that

$$
\text { (1) } 2 S_{t}^{*} \circ T(0, c]= \begin{cases}S_{c-t}-S_{t}^{*}-\int_{0}^{c-t} S_{s} d s-\int_{0}^{t} S_{s}^{*} d s, & 0 \leqq t<c, \\ S_{t-c}^{*}-S_{t}^{*}-\int_{t-c}^{t} S_{s}^{*} d s, & 0 \leqq c<t .\end{cases}
$$


By (1)

$$
\begin{aligned}
\operatorname{LHS}(\mathrm{a})= & \sqrt{ } 2\left\{S_{a}{ }^{*}-I-\int_{0}^{a} S_{t}{ }^{*} d t\right\} T(0, b] \\
= & \left\{S_{b-a}-S_{a}{ }^{*}-\int_{0}^{b-a} S_{s} d s\right\}-\sqrt{ } 2 T(0, b] \\
& \quad-\int_{0}^{a}\left\{S_{b-t}-S_{t}{ }^{*}-\int_{0}^{b-t} S_{s} d s-\int_{0}^{t} S_{s}^{*} d s\right\} d t \\
= & S_{b-a}-S_{a}{ }^{*}-\int_{0}^{b-a} S_{s} d s-\left\{S_{b}-I-\int_{0}^{b} S_{s} d s\right\} \\
& \quad-\int_{0}^{a} S_{b-t} d t+\int_{0}^{a} \int_{0}^{b-t} S_{s} d s d t+\int_{0}^{a} \int_{0}^{t} S_{s}^{*} d s d t .
\end{aligned}
$$

Denoting the last 3 integrals by $J_{1}, J_{2}, J_{3}$, and using the rule of substitution, Fubini's Theorem and Dirichlet's formulae, we find that

$$
\begin{aligned}
& J_{1}=\int_{b-a}^{b} S_{s} d s, \quad J_{3}=\int_{0}^{a}(a-s) S_{s}^{*} d s, \\
& J_{2}=\int_{0}^{b}(b-s) S_{s} d s-\int_{0}^{b-a}(b-a-s) S_{s} d s .
\end{aligned}
$$

Inserting these expressions in (2) and simplifying we get (a). (b) follows on taking adjoints on both sides.

We now turn to the restricted measure $T_{0}(\cdot)$. What concerns us is of course the product $T_{0}(0, b]^{*} T(0, a]$, where ${ }^{*}$ is the adjoint operation for $W$-to-JC (not $\mathfrak{H}$-to- $\mathcal{H C}$ ) linear operators. Obviously,

$$
\forall A \in \mathcal{P}, \quad T_{0}(A)^{*}=P_{W} T(A)^{*}=P_{W} T_{0}(A)^{*},
$$

and therefore

$$
\begin{aligned}
\forall A, B \in \mathcal{Q}, \quad T_{0}(B)^{*} T_{0}(A) & =P_{W} T(B)^{*} T(A) I_{W} \\
& =\text { Rstr. } P_{W} T(B)^{*} T(A) .
\end{aligned}
$$

Combining 15.9 and (11.11), we get

$$
\begin{array}{ll}
2 T_{0}(0, a]^{\#} T_{0}(0, b]=\operatorname{Rstr}_{W} P_{W}\left\{R_{b-a}-R_{a}^{*}-R_{b}\right\}, & 0 \leqq a<b \\
2 T_{0}(0, b]^{\#} T_{0}(0, a]=\operatorname{Rstr} . W P_{W}\left\{R_{b-a}^{*}-R_{a}-R_{b}^{*}\right\}, & 0 \leqq a<b
\end{array}
$$

Next, we appeal to the fundamental result: 
15.13. Lemma (Relation Between $S_{t}$ AND $V^{n}$ ).

(a) $\quad \forall t \geqq 0, \quad S_{t}=e^{-t} I+\operatorname{sim}_{n \rightarrow \infty} \sum_{k=1}^{\infty}\left\{\frac{1}{k !}\left(\frac{-n t}{n+1}\right)^{k} \sum_{j=1}^{k}\left(\begin{array}{c}k \\ j\end{array}\right) K_{n}^{j}\right\}$, where

$$
K_{n}=\frac{2 n}{d}\left\{I-\frac{n-1}{n+1} V\right\}^{-1} V, \quad \text { and so } K_{n}(\mathfrak{H C}) \subseteq V(\mathfrak{H}) .
$$

$$
\forall n \geqq 0, \quad V^{n}=I+2 \int_{0}^{\infty} L_{n}^{\prime}(2 t) e^{-t} S_{t} d t,
$$

where

$$
L_{n}(t)=\sum_{k=0}^{n} \frac{(-1)^{k}}{k !}\left(\begin{array}{l}
n \\
k
\end{array}\right) t^{k}, \quad \text { (nth Laguerre polynomial). }
$$

Proof. The proof is the same as that of the corresponding relations between $U_{t}$ and $V^{k}$, given in [33, Lmas. 2.7, 2.8], where $\left(U_{t}, t \in R\right)$ is a strongly continuous group of unitary operators on HC onto $\mathcal{H C}$ and $V$ is defined as in (15.6)(iii), but with $U_{t}$ instead of $S_{t}$.

The last lemma (a) immediately yields the following crucial theorem:

\subsection{THEOREM.}

(a) $\forall t \geqq 0 \& \forall x \in \mathcal{H}, \quad S_{t}(x)=e^{-t} x+y_{t}, \quad$ where $y_{t} \in V(\mathcal{H C})={ }_{d} W^{\perp}$.

(b) $\quad P_{W} S_{t}=e^{-t} P_{W} \&$ Rstr.W $S_{t}^{*}=e^{-t} I_{W}$.

We can now assert the result we were after:

\subsection{THEOREM.}

(a) $\forall a, b \geqq 0, \quad T_{0}(0, a]^{*} T_{0}(0, b]=\min \{a, b\} I_{W}$.

(b) $T_{0}(\cdot)$ is a W-to-疋, c.a.q.i. measure over $\left([0, \infty), \odot, \mu(\cdot) I_{W}\right)$, where $\mu$ is Lebesgue measure.

Proof. (a) Let $c \geqq 0$. Then from the definition (15.6)(vii) of $R_{c}$ and the first equation in 15.14(b) we get

$$
\begin{aligned}
P_{W} R_{c} & =P_{W}\left\{S_{c}-I-\int_{0}^{c}(c-t) S_{t} d t\right\} \\
& =\left\{e^{-c}-1-\int_{0}^{0}(c-t) e^{-t} d t\right\} P_{W}=-c P_{W} .
\end{aligned}
$$

Similarly, from (15.6)(vii) and the second equation in 15.14(b) we get 


$$
\text { Rstr.W } P_{W} R_{c}^{*}=-c I_{W} .
$$

Combining (1) and (2), we have

$$
\forall c \geqq 0, \quad \text { Rstr. } P_{W} R_{c}=-c I_{W}=\text { Rstr. } P_{W} R_{c}^{*} .
$$

Using the relations (3) and (15.12) we find that for $0 \leqq a<b$

$$
\begin{aligned}
& 2 T_{0}(0, a]^{\sharp} T_{0}(0, b]=\{-(b-a)+a+b\} I_{W}=2 a I_{W}, \\
& 2 T_{0}(0, b]^{\sharp} T_{0}(0, a]=\{-(b-a)+a+b\} I_{W}=2 a I_{W} .
\end{aligned}
$$

This establishes (a).

(b) By $15.7(\mathrm{a}), T_{0}(\cdot)$ is finitely additive on $P$. In addition it satisfies the condition (a). Hence by Lemma 8.12(b) we have (b). that

We turn next to the subspace $S_{T_{0}}$ of the measure $T_{0}$. We first note

$$
S_{T_{0}}=\Im\left\{S_{t}(W): t \geqq 0\right\} .
$$

This follows at once from $\left(15.8^{\prime}\right)$ on setting $X=W$. Next, we notice that the equations in 15.13 (a)\&(b) yield the inclusions $\subseteq$ and $\supseteq$ involved in the following useful identity:

$$
\forall X \subseteq \mathcal{H}, \quad \Im\left\{S_{t}(X): t \geqq 0\right\}=\Im\left\{V^{n}(X): n \geqq 0\right\} .
$$

Finally, we assert a corresponding identity for the "remote" subspaces of the $S_{t-}$ and $V^{n}$-semigroups:

15.18. Theorem. $\mathcal{H}_{\infty}={ }_{d} \bigcap_{t 0} S_{t}(\mathcal{H C})=\bigcap_{n \geq 0} V^{n}(\mathcal{H C})$.

Proof. As mentioned in $[28,3.2]$ a proof can be completed on the basis of the identities in $15.13(\mathrm{a}) \&(\mathrm{~b})$ and the limiting behavior of the Laguerre polynomials $L_{n}(t)$, as $t \rightarrow \infty$. But a shorter proof due to Nagy $[37$, p. 46$]$ is also available.

We can now get our final expression for $\delta_{T_{0}}$, by putting together these fragmentary results and appealing to the discrete Wold decomposition. Thus,

$$
\begin{aligned}
S_{T_{0}} & =\Im\left\{S_{t}(W): t \geqq 0\right\} & & \text { by }(15.16), \\
& =\Im\left\{V^{n}(W): n \geqq 0\right\} & & \text { by }(15.17) \text { with } X=W, \\
& =\sum_{n=0}^{\infty} V^{n}(W), & & \text { by } 15.5, \text { since } V^{n}(W) \text { are } \perp, \\
& =\mathfrak{H C} \cap\left\{\cap V_{n \geqq 0}^{n}(\mathfrak{H C})\right\}^{\perp} & & \text { by } 15.5 \text { (decomposition), } \\
& =\mathfrak{H C} \cap \mathfrak{F C}_{\infty}^{\perp}=\mathfrak{F C}_{0} & & \text { by } 15.18 \&(15.6)(\mathrm{ii}) .
\end{aligned}
$$


By establishing Thm. 15.15 (b) and (15.19) we have completed the hard steps (i) and (ii) of Procedure 12.1. We can now appeal to the general theory in $\S \S 10,11$ to assert our main theorem:

15.20. Theorem (Wold decomposition \& Cooper's Thm.) Under the assumptions (15.6) we have

(a) $T_{0}(\cdot)$ is a $W$-to- $\mathcal{C}_{0}$ basic, c.a.q.i. measure over $\left([0, \infty), \mathbb{B}_{\mu}\right.$, $\left.\mu(\cdot) I_{W}\right)$, where $\mu$ is Lebesgue measure;

(b) $\quad \mathfrak{C}=\mathfrak{K}_{\infty}+\int_{0}^{\infty} T_{0}(d t)(W), \& \mathfrak{H}_{\infty} \perp \int_{0}^{\infty} T(d t)(W)$;

(c) the correspondence $\Sigma: \phi \rightarrow \int_{0}^{\infty} T_{0}(d t) \phi(t)$ is a unitary operator on $L_{2}([0, \infty) ; W)$ onto $\mathfrak{H}_{0}$, such that

$$
\forall t \geqq 0, \quad \operatorname{Rstr}_{\cdot \mathcal{F}_{0}} S_{t}=\Sigma \circ \sigma_{t} \circ \Sigma^{-1},
$$

where $\sigma_{t}$ is as in $15.4(4)$.

(d) $\forall t \geqq 0, \quad R s t r . \mathcal{F C}_{\infty} S_{t}$ is unitary on $\mathfrak{F C}_{\infty}$ onto $\mathfrak{F C}_{\infty}$.

Proof. (a) is just a restatement of 15.15(b) and (15.19).(b) follows from (a) and Cor. 10.9 (a). The first part of (c) follows from (a) and Thm. 10.8. The second part of (c) follows from Thm. 11.10 (a), since by (15.7) (b) the measure $T_{0}$ is stationary with shift semigroup $(S, t \geqq 0)$.

(d) Since $S_{t}$ is an isometry, to prove (d) we have only to show that $S_{t}$ carries $\mathfrak{K}_{\infty}$ onto $\mathfrak{H}_{\infty}$. Let $t \geqq 0$. Since $S_{t}$ is one-one,

$$
S_{t}\left(\mathcal{H C}_{\infty}\right)=S_{d}\left\{\bigcap_{s \geq 0} S_{s}(\mathcal{H C})\right\}=\bigcap_{s \geq 0} S_{s+t}(\mathfrak{H C})
$$

But, since $S_{s+t}(\mathfrak{H C}) \subseteq S_{8}(\mathfrak{H C})$, we have

On the other hand,

$$
\operatorname{RHS}(1) \subseteq \bigcap_{: \geq 0} S_{s}(\mathfrak{H C})=\mathfrak{H}_{\infty}
$$

$$
\operatorname{RHS}(1)=\bigcap_{u \geq t} S_{u}(\mathfrak{F C}) \supseteq \bigcap_{u \geq 0} S_{u}(\mathfrak{F C})=\mathfrak{F}_{\infty} .
$$

By (1)-(3), $S_{t}\left(\mathfrak{H C}_{\infty}\right)=\mathfrak{H}_{\infty}$ as required.

Hitherto we have treated the set $L_{2}(E ; W)$, where $E \subseteq R$, e.g. $E=[0, \infty)$, as comprising functions on $E$ to $W$. In certain cases, e.g. when $S_{t}$ is obtained as in (15.3), it is convenient to redefine this symbol by

(15.21) $L_{2}(E ; W)=\left\{\phi: \phi \in L_{2}(R ; W) \& \phi\right.$ vanishes a.e. on $\left.R \backslash E\right\}$. 
Then by (11.13) the equation in 15.20 (c) can be written

$$
\forall t \geqq 0, \quad \operatorname{Rstr}_{\Im_{0}} S_{t}=\Sigma \mathrm{o} \tau_{-t} \mathrm{O} \Sigma^{-1},
$$

where $\tau_{h}$ is translation through $h$; i.e. $\left(\tau_{h} \phi\right)(t)=\phi(t+h), \quad h, t \in R$. The correspondence $\Sigma^{-1}$ is therefore called the translational representation of the restricted semigroup ( $\operatorname{Rstr.}_{\text {.c }_{0}} S_{t}, t \geqq 0$ ). An application of the FP transformation and the Paley-Wiener Theorem yields a corresponding spectral representation:

15.23. THEOREM (SPECTRAL REPRESENTATION). Let

(i) $\Sigma$ be as in Theorem 15.20(c),

(ii) $\Sigma={ }_{d} \Sigma \circ U$, where $U={ }_{d} \Sigma_{\eta}$ is the FP transformation on $L_{2}(R ; W)$ onto $L_{2}(R ; W), c f .13 .7$,

(iii) $L_{2}^{0+}(R ; W)$ be as in the Paley-Wiener Theorem 13.17.

Then the correspondence $\tilde{\Sigma}$ is a unitary operator on $L_{2}^{0+}(R ; W)$ onto $\mathfrak{H C}_{0}$ such that

$$
\forall t \geqq 0, \quad \operatorname{Rstr}_{\mathfrak{F e}_{0}} S_{t}=\tilde{\Sigma} \circ M_{e t} \circ \tilde{\Sigma}^{-1}, \text { where } e_{t}(\lambda)=\frac{\bar{d}}{=} e^{i t \lambda} .
$$

Proof. By the PW Theorem 13.17, $U$ carries $L_{2}^{0+}(R ; W)$ onto $L_{2}([0, \infty) ; W)$, i.e. by 15.20 (c) onto the domain of $\Sigma$. Hence the domain of $\tilde{\Sigma}={ }_{d} \Sigma \circ U$ is $L_{2}^{0+}(R ; W)$ and its range is that of $\Sigma$, i.e. by 15.20 (c) it is $\mathcal{H}_{0}$. Also, since $U$ and $\Sigma$ are unitary operators, so is $\tilde{\Sigma}$.

Now let $t \geqq 0$, and write $S_{t}^{0}$ for $\operatorname{Rstr} .{ }_{\xi_{0}} S_{t}$. Then by (ii), (15.22), 13.12 and (ii), applied in succession we get

$$
S_{t}^{0} \tilde{\Sigma}=S_{t}^{0} \Sigma U=\Sigma \tau_{-t} U=\Sigma U M_{e_{t}}=\tilde{\Sigma} M_{e_{t}} .
$$

This completes the proof.

When the semigroup $\left(S_{t}, t \geqq 0\right)$ is derived as in (15.3) from a strongly continuous unitary group $\left(U_{t}, t \in R\right)$, the preceding semigroup representations can be extended to yield translational and spectral representations for the group $\left(U_{t}, t \in R\right)$ itself, as shown in $[31, \S 5]$. When applied to the closely related theories of the vectorial Hardy class $\mathrm{H}_{2}$, prediction and scattering, cf. 15.2, these explicit results for $U_{t}$ yield the theorems of Nevanlinna, Beurling, and Lax; the Wold and spectral decompositions of a stationary stochastic process; and the translational and spectral representations of Lax and Phillips [22, p. 7]. This has been indicated in the papers [27], [33], [31], the second of which is with J. B. Robertson.

\section{Unfinished work}

In this section we shall comment on aspects of our theory which have either not been discussed above or discussed perfunctorily. 
16.1. ON THE INTEGRALS $\int_{\Lambda} \Psi(\lambda) M(d \lambda) \Phi(\lambda)$. Our work is affected by the as yet embryonic state of the theory of such integrals in which for $\lambda \in \Lambda \& \Delta \subseteq \Lambda, \Phi(\lambda), M(\Delta), \Psi(\lambda)$ are continuous linear operators, $W^{\prime}$-to- $W, W$-to- $W, W$-to- $W^{\prime \prime}$, respectively. In $\S 9$ we had to define this integral by the different formulae (9.12), (9.17) for two different types of measures $M$. Wanted is a single definition covering all choices of Hilbert spaces $W, W^{\prime}, W^{\prime \prime}$ and all measures $M(\cdot)$. This definition should follow the classical pattern: the integral should first be defined for simple $\Phi, \Psi$, and then by a limiting procedure (say, with respect to the strong operator topology) for arbitrary integrable pairs $(\Phi, \Psi)$. An important interim step would be to treat the integral concept as primitive, and to augment the list of basic properties given in (9.3) into a list of axioms from which all other properties could be deduced. Any proposed definition of the integral would then be deemed "acceptable," if it yielded a realization of the axiomsystem. With an acceptable definition of the integral, the definition (9.5) of $\mathfrak{L}_{2}=L_{2}\left(\Lambda, B, M ; W^{\prime} \circ W\right)^{43}$ would become unambiguous, as would the Criterion 9.9 for the adequacy of $M$.

16.2. Operational calculus. Let $T$ be a $W$-to-秋, c.a.q.i. measure over $(\Lambda, \beta, M)$, $\S$. In $\S 10$ we defined for certain measures $M$ (e.g. adequate ones) the integral

$$
\int_{\Lambda} T(d \lambda)\{\phi(\lambda)\}, \text { where } \phi \text { is on } \Lambda \text { to } W \& \Phi \in \mathfrak{L}_{2, W}
$$

as a vector in $\mathfrak{H C}$. It is also possible to define a related integral

$$
J=\int_{\Lambda} T(d \lambda) \Phi(\lambda), \text { for suitable } \Phi \text { on } \Lambda \text { to } \mathrm{CL}\left(W^{\prime}, W\right),
$$

so that it is a linear operator on $W^{\prime}$-to-秥, by letting

$$
J\left(w^{\prime}\right)=\int_{\Lambda} T(d \lambda)\left\{\Phi(\lambda)\left(w^{\prime}\right)\right\}, \quad w^{\prime} \in W^{\prime},
$$

the integral on the right being of type (1). On the basis of the last definition we can build an operational calculus for quasi-isometric measures somewhat akin to the operational calculus for spectral measures. An especially simple choice of $\Phi(\cdot)$ is $\phi(\cdot) I_{W}$, where $\phi(\cdot)$ is complex-valued. This calculus has already found uses (cf. 16.5), but its full scope remains to be investigated. It would be worthwhile

${ }^{48}$ In which the clause "and is of the trace-class" should be added in case $W^{\prime}$ is infinite dimensional. 
to find out how it relates to the theory of integration of operatorvalued functions with respect to c.a.o.s. measures in the "inflated" tensor product $W^{\prime} \circ W$. In the latter space the inner product of $A$ and $B$ is taken to be the operator ("Gramian") $B^{*} A$ rather than trace $B^{*} A$. Such inflated spaces are important in the study of vectorvalued stochastic processes.

16.3. Pseudo-eigenfunction expansions. The concept of pseudoeigenfunction is usually explicated by going from the initially given Hilbert space $\mathfrak{H C}$ to other spaces, such as generalized function-spaces or rigged Hilbert spaces. Our use of c.a.o.s. measures in $L_{2}$-transform theory (\$\$4-6) and their application to a boundary-value problem of the reduced wave equation given in Appendix II of the Report cited in [30] suggests an alternative approach. In this the pseudoeigenfunctions would be integrated to form eigenpackets, i.e. values of a measure in the Hilbert space $\mathcal{F}$ itself, cf. [16, \$10.4], and this (vector-valued) measure used for purposes of integration. Then it would become unnecessary to leave $\mathfrak{H}$. In future work we hope to demonstrate the efficacy of this approach by bringing it to bear on the work on eigenfunctions of F. I. Mautner [35], W. G. Bade and J. T. Schwartz, and others, cf. [11, II, pp. 1269-1270].

16.4. Perturbation theory. In the so-called stationary approach to perturbation theory due to S. Kuroda, L. de Branges, M. S. Birman and others, cf. references in [21], perturbation theorems are derived from certain spectral representation theorems. In some of these the representation space is a von Neumann direct integral, in others it is the completion of an $L_{2}$-space of vector-valued functions with respect to an operator-valued measure. Differing concepts of operator-measure and vector integration are employed. Certain proofs lean on Hilbert-Schmidt kernels, others on vector-valued holomorphic functions.

The results in $\$ \$ 13-15$, especially 14.15 , suggest that the systematic use of quasi-isometric measures and integration might provide a simpler and more uniform approach to such representation theorems. We hope to investigate this question elsewhere.

16.5. Explicit Form OF the Imprimitivity Theorem. Let $\left(U_{\lambda}, \lambda \in \Lambda\right)$ be a strongly continuous unitary representation of a locally compact group $\Lambda$ on a Hilbert space $\mathfrak{H C}$, $\beta$ be the $\sigma$-algebra generated by the Borel subsets of $\Lambda$ and their complements, and $E(\cdot)$ be a spectral measure for $\mathfrak{H C}$ on $B$ with multiplicity $q \leqq \boldsymbol{\aleph}_{0}$ such that 


$$
\forall \lambda \in \Lambda \& \forall B \in \Theta, \quad E(B+\lambda)=U_{\lambda} E(B) U_{\lambda}^{*} .
$$

Take any H.H. sequence $\left(\beta_{j}, j \in J\right)$ for this $E(\cdot)$, and define the space $W$, the c.a.q.i. measure $E_{0}(\cdot)$ and the hermitian operatorvalued measure $M_{0}(\cdot)$ as in 14.9. Then by Theorem 14.12 the induced unitary operator $\Sigma_{0}$ on $L_{2}\left(\Lambda, \Theta, M_{0} ; W\right)$ onto $\mathcal{H}$ possesses the first intertwining property demanded by the Imprimitivity Theorem (cf. 11.20), viz.

$$
\forall B \in \Theta, \quad E(B)=\Sigma_{0} \circ M_{x_{B} \circ \Sigma_{0}}{ }^{-1} .
$$

$\Sigma$ does not of course possess the second property, viz.

$$
\forall \lambda \in \Lambda, \quad U_{\lambda}=\Sigma_{0} \circ \tau_{-\lambda} \circ \Sigma_{0}^{-1},
$$

for such possession would make the measure $M_{0}$ right-invariant, which is absurd since $M_{0}$ is bounded.

To treat the Imprimitivity Theorem explicitly we will therefore construct from $E_{0}$ and $M_{0}$ new measures $T$ and $N$, such that $N$ is $W^{\prime}$-to- $W^{\prime}$, nonnegative hermitian operator-valued, $T$ is $W^{\prime}$-to-HC, c.a.q.i. over $(\Lambda, \beta, N)$, and the unitary operator $\Sigma$ induced by $T$ has both the desired intertwining properties. In view of Corollary 11.19 (b) for this we need only show that $T$ fulfills the three requirements.

I. $T(\cdot)$ is $W^{\prime}$-to-He basic,

II. $E(\cdot)$ is the spatial integral spectral measure $Q(\cdot)$ of $T(\cdot)$, cf. 10.25(b),

III. $T(\cdot)$ is right-stationary with shift group $\left(U_{\lambda}, \lambda \in \Lambda\right)$.

Space will not permit us to give more than a bare outline of the construction. It hinges on the very simple form assumed by $M_{0}$ in the present situation, as revealed in the following lemma:

Lemma. For the above-mentioned spectral measure $E(\cdot)$, the dominant measure $\mu=\mu_{\beta_{1}}$ of any H.H. sequence and the right-invariant Haar measure $m$ over $\Lambda$ are mutually absolutely continuous. Moreover, $M_{0}(\cdot)=\mu(\cdot) I_{W}$.

The proof, which depends on Mackey's lemma on quasi-invariant measures $[24,3.3]$, will be omitted.

We now proceed heuristically. A little reflection suggests defining $T(\cdot)$ in terms of $E_{0}(\cdot)$ by an integral of the type 16.2(2):

$$
T(B)=\int_{B} E_{0}(d \lambda) \Phi(\lambda), \quad B \in \mathbb{B},
$$


where $\Phi(\cdot)$ is a $W^{\prime}$-to- $W$ operator-valued function, to be chosen so as to secure the requirements I-III. From the calculus of the integrals 16.2(2) and the last lemma we find that

(5) $T(B)^{*} T(A)=N(A \cap B)$, where $N(A)=\int_{A} \Phi^{*}(\lambda) \mu(d \lambda) \Phi(\lambda)$.

We also find that if the operator $\Phi(\lambda)$ is invertible for almost all $\lambda$ ( $\mu$ measure), then $T$ will fulfill the requirements I and II. To determine $\Phi(\cdot)$ further we have therefore to turn to the requirement III.

Let III prevail. Then by Theorem $11.15, N(\cdot)=m(\cdot) H_{0}$. The last lemma suggests that $H_{0}$ should be $I_{W^{\prime}}$, and so we conclude from (5) that

$$
\forall A \in \Theta, \quad m(A) I_{W^{\prime}}=\int_{A} \Phi(\lambda)^{*} \Phi(\lambda) \mu(d \lambda),
$$

i.e.

$$
\Phi^{*}(\lambda) \Phi(\lambda)=(d m / d \mu)(\lambda) \cdot I_{W^{\prime}}, \quad \text { a.e. }(\mu) .
$$

The Polar Decomposition now yields

$$
\Phi(\lambda)=\Psi(\lambda) \cdot \sqrt{ }\{(d m / d \mu)(\lambda)\} \cdot I_{W^{\prime}}, \quad \text { a.e. }(\mu)
$$

where the partial isometry $\Psi(\lambda)$ is, for almost all $\lambda$ ( $\mu$ measure), a unitary operator on $W^{\prime}$ onto $W$ since $\Phi(\lambda)$ is invertible. Thus (4) becomes

$$
T(B)=\int_{B} E_{0}(d \lambda) \sqrt{ }\{(d m / d \mu)(\lambda)\} \Psi(\lambda), \quad B \in \Theta .
$$

Elsewhere we hope to show that a $W^{\prime}$-to- $W$ unitary operatorvalued function $\Psi(\cdot)$ exists for which the $T(\cdot)$ given by (6) satisfies the requirement III.

\section{REFERENCES}

1. W. B. Arveson, Analyticity in operator algebras, Amer. J. Math. 89 (1967), 578-642. MR 36 \#6946.

2. R. G. Bartle, N. Dunford and J. Schwartz, Weak compactness and vector measures, Canad. J. Math. 7 (1955), 289-305. MR 16, 1123.

3. A. S. Besicovitch, $A$ general form of the covering principle and relative differentiation of additive functions. II, Proc. Cambridge Philos. Soc. 42 (1946), 1-10. MR 7, 281.

4. S. Bochner, Inversion formulae and unitary transformations, Ann. of Math. (2) 35 (1934), 111-115.

5. - Harmonic analysis and the theory of probability, Univ. of California Press, Berkeley, Calif., 1955. MR 17, 273. 
6. W. M. Bogdanowicz, Integral representation of linear continuous operators from the space of Lebesgue-Bochner summable functions into any Banach space, Proc. Nat. Acad. Sci. U.S.A. 54 (1965), 351-354, MR $32 \# 4538$.

7. J. L. B. Cooper, One-parameter semigroups of isometric operators in Hilbert space, Ann. of Math. (2) 48 (1947), 827-842. MR 10, 257.

8. H. Cramer, On the theory of stationary random processes, Ann. of Math. (2) 41 (1940), 215-230. MR 1, 150.

9. - A contribution to the theory of stochastic processes, Proc. Second Berkeley Sympos. Math. Statist. and Prob. (1950), Univ. of California Press, Berkeley, Calif., 1951, pp. 329-339. MR 13, 475.

10. J. L. Doob, Stochastic processes, Wiley, New York, 1953. MR 15, 445.

11. N. Dunford and J. Schwartz, Linear operators. I: General theory, Pure and Appl. Math., vol. 7; II: Spectral theory. Selfadjoint operators in Hilbert space, Interscience, New York, 1958; 1963. MR 22 \#8302; MR 32 \#6181.

12. R. E. Edwards and E. Hewitt, Pointwise limits for sequences of convolution operators, Acta Math. 113 (1965), 181-218. MR 31 \#1522.

12a. Y. Fourès-Bruhat and I. E. Segal, Causality and analyticity, Trans. Amer. Math. Soc. 78 (1955), 385-405. MR 16, 1032.

13. P. R. Halmos, Measure theory, Van Nostrand, Princeton, N. J., 1950. MR 11, 504.

14. - Shifts of Hilbert spaces, J. Reine Angew. Math. 208 (1961), 102-112. MR 27\#2868.

15. E. Hellinger, Neue Begrundung der Theorie quadratischer Formen von unendlich vielen Veranderlichen, J. Reine Angew. Math. 136 (1909), 210-271.

16. G. Hellwig, Differential operators of mathematical physics. An introdiction, Springer-Verlag, Berlin, 1964; English transl., Addison-Wesley, Reading, Mass., 1964. MR $29 \# 2682$; MR 35 \#2174.

16a. H. Helson, Lectures on invariant subspaces, Academic Press, New York, 1964. MR 30 \#1409.

17. M. R. Hestenes, Relative self-adjoint operators in Hilbert space, Pacific J. Math. 11 (1961), 1315-1357. MR 25 \#456.

18. E. Hille and R. S. Phillips, Functional analysis and semi-groups, Amer. Math. Soc. Colloq. Publ., vol. 31, Amer. Math. Soc., Providence, R. I., 1957. MR 19, 664.

19. G. Kallianpur and V. Mandrekar, Semi-groups of isometries and the representation and multiplicity of weakly stationary stochastic processes, Ark. Mat. 6 (1966), 319335. MR 34 \#3638.

20. K. Karhunen, Über lineare Methoden in der Wahrscheinlichkeitsrechnung, Ann. Acad. Sci. Fenn. Ser. A.I. 37 (1967).

21. S. T. Kuroda, An abstract stationary approach to perturbation of continuous spectra and scattering theory, J. Analyse Math. 20 (1967), 57-117. MR 35 \#7165.

22. P. D. Lax and R. S. Phillips, Scattering theory, Pure and Appl. Math., vol. 26, Academic Press, New York, 1967. MR 36 \#530.

23. M. Loève, "Fonctions aléatoires du second ordre," in Processes stochastiques et mouvement Brownien (P. Lévy, Editor), Gauthier-Villars, Paris, 1948. MR 10, 551.

24. G. W. Mackey, A theorem of Stone and von Neumann, Duke Math. J. 16 (1949), 313-326. MR 11, 10.

25. - Induced representations of groups and quantum mechanics, Benjamin, New York, 1968.

26. V. Mandrekar and $\mathrm{H}$. Salehi, The square-integrability of operator-valued functions with respect to a non-negative operator valued measure, Michigan State University, Tech. Report, 1969. (To appea: in J. Math. Mech. 20 (1970).) 
27. P. Masani, Shift invariant spaces and prediction theory, Acta. Math. 107 (1962), 275-290. MR 25 \#4344.

28. - Isometric flows on Hilbert space, Bull. Amer. Math. Soc. 68 (1962), 624-632. MR 26 \#2887.

29. - Recent trends in multivariate prediction theory, Multivariate Analysis (P. R. Krishnaiah, Editor) Proc. Internat. Sympos. (Dayton, Ohio, 1965) Academic Press, New York, 1966, pp. 351-382. MR 35 \#5079.

30. - Orthogonally scattered measures, Advances in Math. 2 (1968), 61-117. (Originally, Technical Report \#738, Mathematics Research Center, University of Wisconsin, 1967). MR 37 \#4231.

31. - On the representation theorem of scattering, Bull. Amer. Math. Soc. 74 (1968), 618-624. MR $36 \# 6980$.

32. - "Explicit form for the Fourier-Plancherel transform over locally compact abelian groups," in Abstract spaces and approximation (P. L. Butzer and B. Sz.Nagy Editors), Birkhauser, Basel, 1969, pp. 162-182.

33. P. Masani and J. Robertson, The time-domain analysis of continuous parameter weakly stationary stochastic processes, Pacific J. Math. 12 (1962), 1361-1378. MR 26 \#7047.

34. N. Wiener and P. Masani, The prediction theory of multivariate stochastic processes. I: The regularity condition, Acta Math. 98 (1957), 111-150; II: The linear predictor, Acta Math. 99 (1958), 93-137. MR 20 \#4323; MR 20 \#325.

35. F. I. Mautner, On eigenfunction expansions, Proc. Nat. Acad. Sci. U.S.A. 39 (1953), 49-53. MR 14, 659.

36. V. J. Mizel and K. Sundaresan, Representation of additive and biadditive functionals, Arch. Rational Mech. Anal. 30 (1968), 102-126. MR 37 \#3341.

37. B. Sz.-Nagy, Isometric flows in Hilbert space, Proc. Cambridge Philos. Soc. 60 (1964), 45-49. MR 28 \#2448.

38. M. A. Nałmark, Normed rings, GITTL, Moscow, 1956; English transl., Noordhoff, Groningen, 1964. MR 19, 870; MR 34 \#4928.

39. R. E. A. C. Paley and N. Wiener, Fourier transforms in the complex domain, Amer. Math. Soc. Colloq. Publ., vol. 19, Amer. Math. Soc., Providence, R. I., 1934; reprint 1967.

40. R. R. Phelps, Lectures on Choquet's theorem, Van Nostrand, Princeton, N. J., 1966. MR 33 \#1690.

41. F. Riesz and B. Sz. -Nagy, Functional analysis, Akad. Kiad6, Budapest, 1953; English transl., Ungar, New York, 1955. MR 15, 132; MR 17, 175.

42. Ju. A. Rozanov, Spectral analysis of abstract functions, Teor. Verojatnost. i Primenen. (1959), 291-310, 4 = Theor. Probability Appl. 4 (1959), 271-287. MR $22 \# 8565$.

43. - Stationary random processes, Fizmatgiz, Moscow, 1963; English transl., Holden-Day, San Francisco, Calif., 1967. MR 28 \#2580; MR 35 \#4985.

44. M. Rosenberg, The square-integrability of matrix-valued functions with respect to a non-negative Hermitian measure, Duke Math J. 31 (1964), 291-298. MR 29 \#649.

45. I. E. Segal and R. A. Kunze, Integrals and operators, McGraw-Hill, New York, 1968. MR 36 \#335.

46. I. Singer, Linear functionals on the space of continuous mappings of a compact Hausdorff space into a Banach space, Rev. Math. Pures Appl. 2 (1957), 301-315. (Russian) MR 20 \#3445.

47. - Les duals de certains espaces de Banach de champs de vecteurs. I, II, Bull. Sci. Math. (2) 82 (1958), 29-40; (2) 83 (1959), 73-96. MR 20 \#3447; MR 22 \#9840. 

199.

48. G. N. Watson, General transforms, Proc. London Math. Soc. 35 (1933), 156-

49. N. Wiener, Differential space, J. Math. and Phys. 2 (1923), 131-174.

50. - The Fourier integral and certain of its applications, Cambridge Univ. Press, Cambridge, 1933.

51. A. M. Yaglom, Certain types of random fields in n-dimensional space similar to stationary stochastic processes, Teor. Verojatnost. i Primenen. 2 (1957), 292-338= Theor. Probability Appl. 2 (1957), 273-319. MR 20 \#1353.

Indiana University, BloOMington, Indiana 47401 AND Battelle Seattle Research Center, Seattle, Washington $98105^{44}$

44 Sincere thanks are due to the staff of the BSR Center for the preparation of this report and of transparencies of the original abridged version, and to Miss Linda Holby in particular for the immaculate typing of the original manuscript. 